\title{
Bayesian Inference Using JET's Microwave Diagnostic System
}

\author{
S. Schmuck, ${ }^{1,2, a)}$ J. Svensson, ${ }^{3}$ L. Figini, ${ }^{1}$ D. Micheletti, ${ }^{1}$ and R. B. Morales ${ }^{2}$ \\ 1) Istituto per la Scienza e Tecnologia dei Plasmi, CNR, via Cozzi 53, 20125 Milan, Italy \\ ${ }^{2)}$ CCFE, Culham Science Centre, Abingdon, OX14 3DB, UK \\ 3) Max-Planck-Institut für Plasmaphysik Teilinstitut Greifswald, Wendelsteinstraße 1, D-17491 Greifswald, Germany
}

\begin{abstract}
At the JET tokamak, three electron cyclotron emission (ECE) diagnostics (two Martin-Puplett interferometers and a heterodyne radiometer) and a reflectometer form the basic microwave diagnostic system. The standard analysis approaches deduce electron density and temperature profiles independently of each diagnostic measurement. Via the Bayesian framework Minerva, the microwave diagnostic system is modelled, and electron temperature and density profiles are inferred jointly for an Ohmic JET plasma. Furthermore, profile length-scales for different plasma domains, a magnetic field correction, distinct reflection properties of the high-field side and low-field side walls, and radiometer sensitivities are estimated together. This inference scheme can use one of two models to predict broadband ECE spectra; one is less accurate but fast for a single prediction, and a more accurate model, relying on the ray-tracer SPECE parallelised via web services. The faster model allows the investigation of correlations between parameters and the execution of a numerical marginalisation, i.e., an uncertainty propagation.
\end{abstract}

\section{INTRODUCTION}

During the 1950's, designs of experimental devices were developed to sustain nuclear fusion processes inside a plasma medium by applying an externally driven magnetic field. This led independently to the first fusion devices built like the stellarator Model $\mathrm{A}^{1}$ and the tokamak T- $1^{2}$ and their corresponding successors. Since then, technological and scientific progress has been made towards a fusion power plant also due to joint undertakings, i.e., international collaborations ${ }^{2,3}$. Along with this progress, the demand is increasing constantly for suitable diagnostic applications to obtain information about parameters of the plasma and of machine components. As a consequence, dozens of diagnostics acquire data simultaneously during discharges in modern fusion experiments, like the Joint European Torus ${ }^{4}$ (JET) tokamak and the stellarator Wendelstein 7- $\mathrm{X}^{5}(\mathrm{~W} 7-\mathrm{X})$. The number of diagnostic applications is likely to rise even further for the future fusion machines ITER ${ }^{6}$ and $\mathrm{DEMO}^{7}$.

For decades, fusion research has been accompanied by the development of diverse diagnostic approaches and components, summarised in Ref. ${ }^{8}$ for the electron cyclotron emission (ECE) instruments. Besides any hardware aspect, the development of data analysis approaches and tools is of the utmost importance to investigate and test physics hypotheses and predictive models. However, this testing remains challenging for a complex problem like a magnetised fusion plasma medium, consisting of many particle species, and its related multitude of physics phenomena, acting on different temporal and spatial scales. In addition, aspects like working principles, absolute sensitivities, and measurement uncertainties of different diagnostics increase the complexity.

With the progress in available computational resources, more complex problems can be investigated nu-

a)stefan.schmuck@istp.cnr.it merically. Though, the complexity of a detailed plasma physics model and diverse diagnostics models is still too large for a joint analysis scheme using up to date computational infrastructures. For example, the inter-pulse analysis at JET carries out a chain of about 100 individual analysis steps ${ }^{9}$, each evaluating certain physics quantities like kinetic profiles of a particle species or magnetic field strength from data measured by the corresponding individual diagnostic. Although, the physics quantities are not derived by a joint estimation but in a hierarchical way, relying on other input quantities. Furthermore, the physics quantities are deduced disjointly with respect to time, and, thus, the dynamical plasma behaviour is left out entirely. In total, the inter-pulse analysis has the advantage of obtaining rough first results in a timely manner, but the disadvantages are manifold. If evaluated, any uncertainty, systematic- and measurement-wise, is not propagated on the results downstream the hierarchical approach. Missed systematics effects linked to a specific diagnostic could lead to unnoticed inconsistencies from a plasma physics point of view. In addition, excluded but important features for a given diagnostic or physics model might not be detectable without a joint approach, relying on measurements of independent diagnostics. Finally, the confidence in the derived description of the plasma might be unclear or unknown. As a consequence, the implications are seldomly studied for when the inter-pulse analysis results are used by refined physics models like JINTRAC ${ }^{10}$ and TRANSP ${ }^{11}$ to estimate essential dynamical quantities like transport coefficients, fluxes and confinement times.

From a scientific point of view, a joint, time- and/or diagnostic-wise, analysis scheme is preferential. Such a combined approach leads automatically to systematic and measurement uncertainties, so that predictions of the model used become objectively consistent with measured data for a certain set of model parameters. In addition, uncertainties on these parameters and their correlations can be inferred. Bayes' theorem offers the possibility for a joint inference, and even competing models could be 
tested for their plausibility. Due to its probabilistic nature, however, Bayesian inference usually demands a lot of computational power and, thus, is not applied for practical reasons. Nevertheless, some progress has been made in this direction. For example, kinetic profiles have been reconstructed and tested for consistency with predictions of heat transport models ${ }^{12,13}$, although omitting diagnostic models. Individual diagnostic models have been developed and in cases combined in applications at ASDEX Upgrade $^{14,15}$, Wendelstein 7-AS ${ }^{16,17}$, JET ${ }^{18-20}$, Alcator C-Mod ${ }^{21}$,Wendelstein $7-\mathrm{X}^{22,23}$ to infer for instance kinetic profiles, the flux surface geometry, the current profile and/or diagnostic calibration including uncertainties.

Some of the aforementioned applications have been carried out inside the Bayesian framework Minerva ${ }^{24}$ which enables a joint probabilisitic inference scheme. Minerva offers non-experts the possibility to benefit from probabilistic facets, plasma physics and diagnostic models, and numerical methods each implemented by experts in the corresponding fields. This work adds to Minerva a generalised squared-exponential prior covariance, two ECE models and three diagnostic models. The generalised squared-exponential covariance allows the estimation of different length-scales, governing for example kinetic profiles in the plasma core and edge domains. Furthermore, the location of the transition, separating core and edge domains, can be inferred.

The ECE models predict broadband spectra, covering several harmonic ranges, available at a diagnostic antenna. The first ECE model, i.e., a parallelised version of the ray-tracer SPECE is more accurate physics-wise but also costly from a computational point of view. To overcome this difficulty, the alternative model ECEPT was developed which is sufficiently accurate for Ohmic plasma conditions and for a standard vacuum diagnostic line of sight. Both ECE models share the assumption that the reflection properties, considered as free parameters, of the high-field side (HFS) wall and the low-field side (LFS) wall differ. This assumption and multi-reflections at the walls enhance the wave amplitudes dependent on the harmonic range and especially affect spectral domains for which the plasma is optically thin.

The three diagnostic models capture the fundamental principles of a Martin-Puplett interferometer, a heterodyne radiometer and a reflectometer, respectively. For the interferometer, a probed broadband spectrum has undergone a spectral convolution. A linear model predicts the data acquired with the radiometer, having the channel dependent sensitivity as a free parameter. For the reflectometer, effective data in terms of optical path difference are predicted in a demerged manner for each spectral band.

The added Minerva features are used in the example application of an Ohmic JET plasma. This application estimates jointly electron density and temperature profiles, a magnetic field correction, wall properties, and radiometer channel sensitivities given measurements of the standard microwave diagnostic system at JET.
In Section II, the Bayesian model, which is used to perform the example application, is abstractly overviewed. The interested reader benefits from the comprehensive Sections III-V and Appendices A-E which present details of the used models for the plasma, ECE spectra, diagnostics, and diverse side aspects. The example application can be found in Sec. VI, and the final Section concludes.

\section{BAYESIAN MODEL}

After a brief introduction to Bayes' theorem, basic aspects of both Bayesian models are presented. These models are implemented in the framework Minerva and are subdivided here into the plasma models, ECE spectra and diagnostic models, and relativistic X-mode cut-off and reflectometer models. To each submodel a section is dedicated, providing more insights.

\section{A. Bayes' Theorem}

If the free parameters summarised by the set $\vec{P}$ can be interpreted as continuous random variables, then the knowledge about these parameters is expressed by a joint probability density. While the knowledge is stated by the prior $p(\vec{P})$, for instance a uniform or a multi-variate normal distribution, before a new data set $\vec{D}$ is available, the updated knowledge is given by the posterior $p(\vec{P} \mid \vec{D})$ after new evidence is at hand. According to Bayes' theorem ${ }^{25}$

$$
p(\vec{P} \mid \vec{D})=\frac{p(\vec{D} \mid \vec{P})}{p(\vec{D})} p(\vec{P}),
$$

both probability densities are connected via two additional probability densities; the likelihood $\mathcal{L}=p(\vec{D} \mid \vec{P})$, and the evidence $p(\vec{D})$. The evidence is a constant and is of no further interest for a given model.

The likelihood quantifies in probabilistic terms the mismatch between predictions, following from the free parameters mapped by a physics model for example, and data. For instance, a multi-variate normal (Gaussian) likelihood demands a data covariance, according to the measurement uncertainties, and, thus, smaller uncertainties increase the probabilistic mismatch between given prediction and data.

For a complex problem, a quasi-continuous quantity like a temperature profile needs to be inferred. Such a quantity might have a certain length-scale with respect to the relevant coordinate. This length-scale appears in the joint prior of the temperature profile parameters but does not enter explicitly in the likelihood. However, the Bayesian approach allows an estimation for this kind of parameter, when a dedicated prior is assigned to the quasi-continuous quantity.

For a problem, distinct parameter sets $\vec{P}_{1}, \vec{P}_{2}, \ldots$, linked to the temperature profile and density profile for 


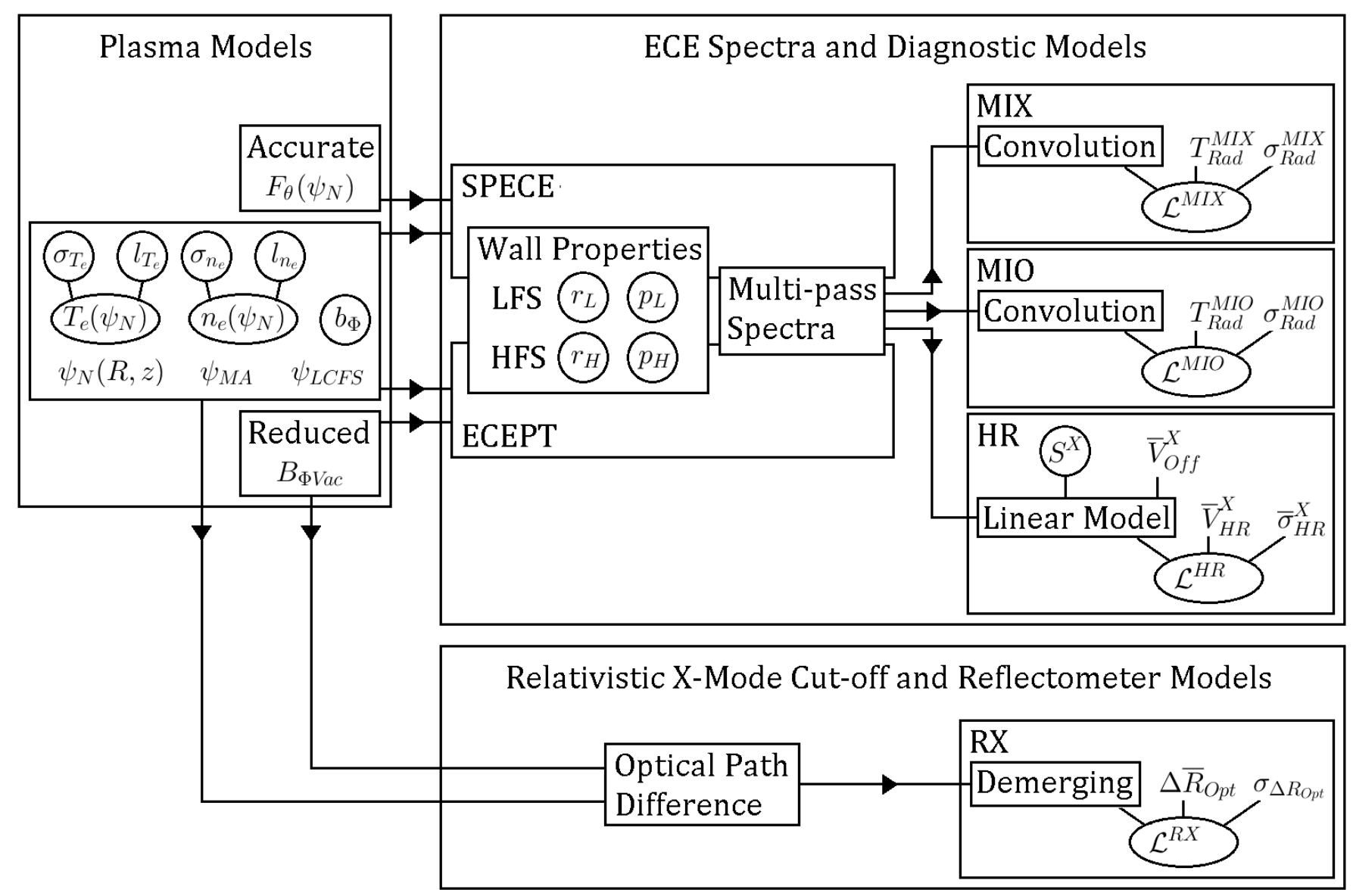

Figure 1. Scheme of Minerva Bayesian model subdivided into plasma models (one more accurate), ECE spectra (more accurate model relies on SPECE) and diagnostic models (interferometers MIX and MIO, and heterodyne radiometer HR), relativistic $\mathrm{X}$-mode cut-off and reflectometer (RX) models. Circles and ellipses indicate probabilistic entities of the model. Quantities are passed across submodels indicated by black arrows. For example, plasma equilibrium quantities, like the normalised poloidal flux surface geometry $\psi_{N}(R, z)$, enter in SPECE or ECEPT to compute multi-pass spectra probed by MIX, MIO and HR. The free parameters of the problem are the electron temperature $T_{e}\left(\psi_{N}\right)$ and density $n_{e}\left(\psi_{N}\right)$ profiles, profile absolute scalings $\sigma_{T_{e}}$ and $\sigma_{n_{e}}$, profile length-scales $l_{T_{e}}$ and $l_{n_{e}}$, the correction $b_{\Phi}$ to the vacuum toroidal magnetic field, wall reflection properties $r_{L}, p_{L}, r_{H}, p_{H}$, and the linear sensitivity $S^{X}$ for each X-mode radiometer channel. Considered diagnostic principles are the spectral convolution for MIX and MIO, the linear response for HR, and the demerging (independent spectral bands) for RX. Predictions and effective data sets $T_{R a d}^{M I X}, T_{R a d}^{M I O}, \bar{V}_{H R}^{X}$ and $\Delta \bar{R}_{O p t}$, all four derived from actual measurements, are related to each other via the Gaussian likelihoods $\mathcal{L}^{M I X}, \mathcal{L}^{M I O}, \mathcal{L}^{H R}$, and $\mathcal{L}^{R X}$, respectively. To finalise the likelihoods, corresponding uncertainties have been derived.

instance, are to be inferred simultaneously. If no joint prior information is available, the prior can be factorised by $p(\vec{P})=\prod_{i} p\left(\vec{P}_{i}\right)$.

The Bayesian ansatz can be applied also to effective data derived from actual measured data by some operations. In this case, it is essential to propagate the uncertainties from the data domain to the effective data domain. Furthermore, only if all effective data sets, relying on measurements of distinct diagnostics, remain independent from each other after operations have been carried out, the joint likelihood factorises like $\mathcal{L}=\prod_{i} \mathcal{L}^{\text {Diagnostic } i}$
Even if the right-hand side of Bayes' theorem can be stated easily with $\mathcal{L}$ and $p(\vec{P})$, the posterior investigation might imply intensive computational efforts, especially when the problem has no analytical solution and/or the number of free parameters and data points becomes large. For example, Markov chain Monte Carlo (MCMC) sampler have been developed to explore the parameter domain for plausibility.

\section{B. Minerva}

The framework Minerva allows a user to capture probabilistic and deterministic aspects of physics and diagnostic models, using linked graphical models. This is 
shown abstractly by figure 1 for the example presented in this work. The probabilistic part relies on Bayes' theorem, taking into account prior information as well as the uncertainty of the deterministic, i.e., predictive model stated by the likelihood. The deterministic part is summarised by a set of linked computational blocks. Each block mimics a certain physics aspect and evaluates from provided input quantities like free parameters the output quantities, being inputs to other blocks. At some point, a physics feature is modelled which enters the corresponding diagnostic model implemented as a set of blocks by corresponding experts. Consequently, predictions can be made for specific values of the free parameters of the physics and diagnostic models.

Predictions are objectively compared with measured (raw) data and/or effective (pre-processed) data via an appropriate likelihood, considering systematic and/or measurement uncertainties. Furthermore, prior information about the free parameters must be assigned inside the framework. Minerva offers the usage of common priors like uniform or multi-variate distributions. For instance, kinetic profiles to be inferred can be treated a priori as Gaussian processes whose covariances enable the estimation of length-scales as well.

For an implemented problem, Minerva provides numerical tools, like optimisers, to find the maximum of the posterior, MCMC sampler and linear Gaussian inversion methods to investigate the joint posterior of the free parameters.

\section{Model Overview}

Abstractly, the Bayesian model is presented by the scheme shown in figure 1. Actually, two models are used; one is more accurate with respect to the plasma submodel and the ECE submodel. However, the accurate version has an extended computational duration per prediction which excludes MCMC sampling.

Each Bayesian model compares predictions, depending on the free parameters like electron density and temperature profiles, with effective data sets, taking into account prior information. The effective data sets have been deduced from measurements which are acquired independently by four diagnostics. Hence, the joint likelihood $\mathcal{L}=\mathcal{L}^{M I X} \mathcal{L}^{M I O} \mathcal{L}^{R X} \mathcal{L}^{H R}$ is given by the product of four likelihoods separately derived for each diagnostic.

\section{Plasma Models}

With its implications, the plasma is assumed to be in a stable axisymmetric equilibrium state captured by the Grad-Shafranov equation. This equilibrium itself is not inferred in this work, but the solution for the flux surface geometry $\psi(R, z)$ dependent on major radius $R$ and height $z$ is taken from the standard equilibrium reconstruction at JET. While the poloidal magnetic field com- ponent follows from $\psi(R, z)$, the toroidal magnetic field component is computed in two ways. The accurate one uses the poloidal current function $f_{\theta}$ associated with the Grad-Shafranov equation, and the alternative combines the vacuum magnetic field $B_{\Phi V a c}$ and the $1 / R$ dependency. For both methods, the free parameter $b_{\Phi}$ changes the vacuum field, and its prior covers a reasonable domain.

The electron density profile $n_{e}$ and electron temperature profile $T_{e}$ depend on the normalised flux surface label $\psi_{N}$ and, therefore, are preserved on a given flux surface like the pressure. Each profile is modelled as a Gaussian process, and the associated prior is a multivariate normal distribution for the chosen parameterisation. The chosen prior covariance may have free parameters itself like, for instance, an absolute scaling $\sigma_{T_{e}}\left(\sigma_{n_{e}}\right)$ and at least one length-scale $l_{T_{e}}\left(l_{n_{e}}\right)$. These covariance parameters have each a uniform prior over a meaningful domain.

\section{ECE Spectra and Diagnostics Models}

For given plasma parameters, one of two ECE models, SPECE and ECEPT, predicts a broadband multipass spectrum at a diagnostic antenna. The ray-tracer SPECE in combination with the more precise plasma model captures more accurately the underlying physics like, for instance, refraction, emission and absorption processes. However, this accurateness is accompanied by a long evaluation time per single prediction, such that an investigation of the posterior cannot be achieved practically. To allow the investigation of the posterior to some degree, a parallelisation was implemented, relying on a multitude of SPECE web services.

For the standard observation of a tenuous and lowtemperature plasma and for the less accurate plasma model, a reduction of the ECE model accurateness is possible which leads to ECEPT. This model neglects refraction, uses a low order approximation for the relativistic effect, and focusses on approximations for emission, absorption and relevant cut-offs for extra-ordinary $(\mathrm{X})$ and ordinary $(\mathrm{O})$ mode waves, respectively. ECEPT predicts a spectrum much faster than SPECE, and, thus, is suitable for the detailed investigation of the posterior when an Ohmic plasma is modelled.

The multi-pass spectrum is modelled by SPECE and ECEPT, assuming that reflection properties of the LFS and HFS inner wall are separate quantities. These properties are estimated by the coefficients $r_{L}$ (reflected divided by incident wave amplitude) and $p_{L}$ (fractional amplitude converted from one polarisation direction to the other) for the LFS wall and their counterparts $r_{H}$ and $p_{H}$. These four free parameters can take values between 0 and 1 , limiting each uniform prior.

SPECE and ECEPT take into account a mismatch, related to the finite pitch angle of the magnetic field at the plasma edge, between antenna orientation and $\mathrm{X}$ - 
and O-mode polarisation directions of the incident waves. Due to the mismatch, a linear combination of spectra in $\mathrm{X}$ - and O-mode polarisations contributes to the detected signal.

Predictions are performed for two Martin-Puplett interferometers MIX and MIO and a multi-channel heterodyne radiometer HR, considering for each the basic measurement principle. For the interferometers, broadband spectra predicted by SPECE or ECEPT undergo a spectral convolution operation linked to the probing of a finite amount of Fourier coefficient inherent to this diagnostic type. The predicted convoluted spectra $T_{R a d}^{M I X}$ and $T_{R a d}^{M I O} \mid$ enter the Gaussian likelihoods $\mathcal{L}^{M I X}$ and $\mathcal{L}^{M I O}$ and are compared with the effective data sets $T_{R a d}^{M I X}$ (main contribution from X-mode spectrum) and $T_{R a d}^{M I O}$ (main contribution from O-mode spectrum) in terms of radiative temperature, respectively. The mandatory spectral uncertainties $\sigma_{\text {Rad }}^{M I X}$ and $\sigma_{\text {Rad }}^{M I O}$ have been investigated and estimated.

Besides SPECE or ECEPT predictions for each Xmode channel, the radiometer model demands the linear responsivity (sensitivity) $S^{X}$, being a free parameter, a voltage offset $\bar{V}_{O f f}^{X}$ and the measurement uncertainty $\bar{\sigma}_{H R}^{X}$. After having estimated the latter two quantities from measured data, the predicted data $V_{H R}^{X} \mid$ and the temporal average $\bar{V}_{H R}^{X}$ of the measured data are related to each other via the Gaussian likelihood $\mathcal{L}^{H R}$. The prior of each sensitivity $S^{X}$ is chosen to be Gaussian with large variance which itself is not of further interest.

\section{Relativistic X-Mode Cut-off and Reflectometer Models}

An optical-path-difference-like quantity, varying with probing frequency, is predicted for given plasma parameters and for the standard observation of the plasma. The prediction is carried out separately for each spectral band of the X-mode reflectometer which keeps the demerged measurement principle. Furthermore, predictions follow from the spatial integration of the local refractive index for the X-mode up to the cut-off position, and a sufficient approximation of the relativistic effect is made.

Probabilistically, the Gaussian likelihood $\mathcal{L}^{R X}$ weighs the difference between predictions $\Delta R_{O p t} \mid$ and effective data $\Delta \bar{R}_{O p t}$ obtained for each band. $\Delta \bar{R}_{O p t}$ has contributions from the reference phase, the phase measurement during plasma operation, and a spectral ramp. The slope of this ramp is determined by the band-dependent reflection location for the reference measurement at the inner wall on the HFS of JET. The uncertainties are propagated from the three contributions onto the effective data, and $\mathcal{L}^{R X}$ is finalised.

\section{Joint and Marginal Posteriors}

For kinetic profiles having each one length-scale, the joint posterior is written out as

$$
\begin{aligned}
& p\left(T_{e}\left(\psi_{N}\right), \sigma_{T_{e}}, l_{T_{e}}, n_{e}\left(\psi_{N}\right), \sigma_{n_{e}}, l_{n_{e}}, b_{\Phi}, r_{L}, r_{H}, p_{L}, p_{H}, S^{X}\left(f_{H R}\right) \mid T_{R a d}^{M I X}\left(f^{\prime}\right), T_{R a d}^{M I O}\left(f^{\prime}\right), \bar{V}_{H R}^{X}\left(f_{H R}\right), \Delta \bar{R}_{O p t}\left(f_{R X}^{\prime}\right)\right) \\
& =\frac{\mathcal{L}^{M I X} \mathcal{L}^{M I O} \mathcal{L}^{R X} \mathcal{L}^{H R}}{p\left(T_{\text {Rad }}^{M I X}, T_{\text {Rad }}^{M I O}, \bar{V}_{H R}^{X}, \Delta \bar{R}_{O p t}\right)} p\left(T_{e}, \sigma_{T_{e}}, l_{T_{e}}\right) p\left(n_{e}, \sigma_{n_{e}}, l_{n_{e}}\right) p\left(b_{\Phi}\right) p\left(r_{L}\right) p\left(r_{H}\right) p\left(p_{L}\right) p\left(p_{H}\right) p\left(S^{X}\right) \\
& \propto \exp \left[-\frac{1}{2} \sum_{f^{\prime}} \frac{\left(T_{R a d}^{M I X}-T_{R a d}^{M I X} \mid\right)^{2}}{\left(\sigma_{R a d}^{M I X}\right)^{2}}\right] \exp \left[-\frac{1}{2} \sum_{f^{\prime}} \frac{\left(T_{R a d}^{M I O}-T_{R a d}^{M I O} \mid\right)^{2}}{\left(\sigma_{\text {Rad }}^{M I O}\right)^{2}}\right] \exp \left[-\frac{1}{2} \sum_{\text {Bands } f_{R X}^{\prime}} \frac{\left(\Delta \bar{R}_{O p t}-\Delta R_{O p t} \mid\right)^{2}}{\sigma_{\Delta R}^{2}}\right] \\
& \times \exp \left[-\frac{1}{2} \sum_{f_{H R}} \frac{\left(\bar{V}_{H R}^{X}-V_{H R}^{X} \mid-\bar{V}_{O f f}^{X}\right)^{2}}{\left(\bar{\sigma}_{H R}^{X}\right)^{2}+\left(\bar{\sigma}_{O f f}^{X}\right)^{2}}\right] \frac{1}{p\left(T_{R a d}^{M I X}, T_{R a d}^{M I O}, \bar{V}_{H R}^{X}, \Delta \bar{R}_{O p t}\right)} \\
& \times p\left(T_{e} \mid \sigma_{T_{e}}, l_{T_{e}}\right) p\left(\sigma_{T_{e}}\right) p\left(l_{T_{e}}\right) p\left(n_{e} \mid \sigma_{n_{e}}, l_{n_{e}}\right) p\left(\sigma_{n_{e}}\right) p\left(l_{n_{e}}\right) p\left(b_{\Phi}\right) \prod_{f_{H R}} p\left(S^{X}\right) .
\end{aligned}
$$

Note, Minerva composes the above expression automatically, including the normalisation constants for the likelihoods which have been omitted here.

The joint prior has been factorised manifold. A priori, $T_{e}$ and $n_{e}$ profiles are independent from each other, and the multi-variate normals $p\left(T_{e}\left(\psi_{N}\right) \mid \sigma_{T_{e}}, l_{T_{e}}\right)$ and $p\left(n_{e}\left(\psi_{N}\right) \mid \sigma_{n_{e}}, l_{n_{e}}\right)$ are multiplied with uniform priors over reasonable domains for the absolute scaling and length-scale, respectively. Individual priors for the reflec- tion properties are chosen to be uniform for the interval $[0,1]$ which leads to unity for each. As starting point, the channel sensitivities $S^{X}\left(f_{H R}\right)$ can be considered to be independent, and the product of individual normal distributions forms their prior.

The joint posterior peaks globally for parameter sets which give good agreement between predictions and data sets and are in accordance with prior information. To remain close to the main peak, only certain parameter 
combinations are entitled which implies present or absent correlations. Causes for considerable correlations can be investigated and should be identified to guide the scientific understanding.

The confidence about an individual parameter, say $r_{L}$, is obtained from the shape of the marginal posterior $p\left(r_{L} \mid T_{R a d}^{M I X}, T_{R a d}^{M I O}, \bar{V}_{H R}^{X}, \Delta \bar{R}_{O p t}\right)$, demanding the marginalisation with respect to all other parameters.

\section{PLASMA MODELS}

This work relies on a plasma medium, being in a stable magnetohydrodynamics (MHD) equilibrium state. Many implications of this major assumption can be found in $^{26}$, like-wise for the ideal MHD equilibrium model. For the theoretical derivation of this idealised case, some aspects are described briefly for the sake of completeness.

For an axisymmetric fusion device like the JET tokamak, the Grad-Shafranov equation expresses the final form of the ideal MHD equilibrium model. The plasma equilibrium itself is not inferred probabilistically like in Ref. ${ }^{18}$. Instead, quantities like the flux surface geometry and the poloidal current function are taken from results of the standard equilibrium reconstruction at JET. Though, a correction to the toroidal vacuum magnetic field is to be estimated. For later purposes, two ways are considered to evaluate the toroidal magnetic field component.

Electron kinetic profiles and their parameterisation are modelled by Gaussian processes. The corresponding multi-variate normal prior distributions enable the choice of a suitable prior covariance. In addition, a covariance has free parameters like a length-scale, for instance.

\section{A. Implications of Axisymmetric Ideal MHD Equilibrium}

A fusion plasma confined to some degree by a magnetic field is a conglomerate of charged particle species. Theoretically, the electro-magnetic aspects are captured by Maxwell equations, and the dynamical behaviour of each particle distribution function may be stated by a Boltzmann equation with diverse sink and source terms. Altogether, a large set of coupled partial differential equations governs the physics over small and large temporal and spatial scales. More unknown quantities exist than equations are at hand, and, thus, an analytical solution of the full problem cannot be achieved from a theoretical point of view. Such a situation can be approached in two ways, at least. The reduction of the model complexity is an option, or experimental data could be used to diminish the degrees of freedom of the problem. The former approach is usually followed, when the plasma equilibrium state is of interest on the time scale of milliseconds.

The complexity is reduced tremendously by many assumptions and restrictions like, for example, each Boltzmann equation includes a Coulomb-collision term but no additional term (for diffusion, auxiliary heating power, neutral gas,...), only lowest order velocity moments of each particle distribution function are considered, isotropic pressure and a Maxwellian distribution function are assumed for each particle species, a quasineutral two-fluid plasma is assumed made from electrons and singly-charged ions, the one-fluid plasma is obtained for a thermal equilibration between electrons and ions, and the resistivity is negligible. As a result, an equation set remains which marks the ideal $\mathrm{MHD}^{26}$ model.

For the idealised MHD model, the force-free, static and axisymmetric plasma equilibrium is captured by the Grad-Shafranov equation ${ }^{27}$. This partial differential equation has three free functions; the poloidal flux surface geometry $\psi(R, z)$ as a function of the major radius $R$ and the height $z$, the derivative of the total plasma pressure $p^{\prime}(\psi)=d p / d \psi$, and the poloidal current function $f_{\theta}(\psi)$. Thereby, $p$ and $f_{\theta}$ are flux functions, and $f_{\theta}=R B_{\Phi} / \mu_{0}$ is linked with the local toroidal magnetic field $B_{\Phi}$ divided by the vacuum permeability. The poloidal magnetic field component $B_{\theta}$ can be derived from $\psi(R, z)$.

Finding a solution of the Grad-Shafranov equation demands further assumptions, like boundary conditions, and/or constraints ${ }^{28-31}$. For example, diverse measurements, constraining $p$ and/or $f_{\theta}$, guide a so-called equilibrium reconstruction procedure to find a suitable flux surface geometry.

\section{B. Assumed Equilibrium Quantities}

The flux surface geometry, the poloidal current function and the vacuum toroidal magnetic field are used, being derived from the standard equilibrium reconstruction $\left(\mathrm{EFIT}^{30}\right)$ at JET. This reconstruction, carried out for instance during the inter-pulse phase, makes use of measurements of magnetic diagnostics to constrain only $f_{\theta}$ to some degree ${ }^{31}$. For a chosen parameterisation of $\psi(R, z)$ and $p(\psi)$, the Grad-Shafranov equation is solved numerically. Hence, this standard reconstruction solves also for the plasma pressure but systematic deviations from the actually measured counterpart can be found. To minimise any systematic effect not included in the specific equilibrium reconstruction, an Ohmic plasma with low density and temperature should be considered, so that the plasma pressure contribution remains small indeed.

\section{Poloidal Flux Surface Geometry}

The poloidal flux surface geometry $\psi(R, z)$ is used shown in figure $2(\mathrm{a})$. From $\psi(R, z)$, the poloidal magnetic field component $B_{\theta}$ can be evaluated ${ }^{27}$. Furthermore, the common way expresses kinetic profiles depending on the normalised flux coordinate $\psi_{N}$. The corresponding conversion is achieved via $\psi_{N}(R, z)=\left(\psi-\psi_{M A}\right) /\left(\psi_{L C F S}-\psi_{M A}\right)$ with the fluxes 
(a)

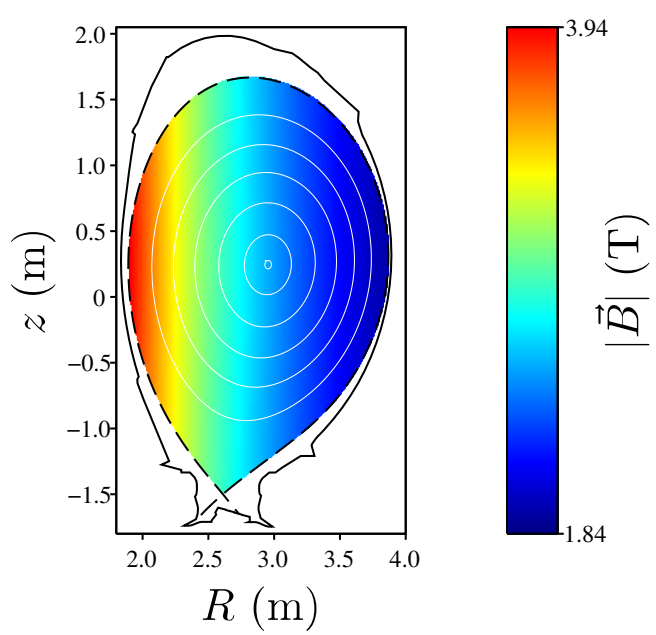

(b)

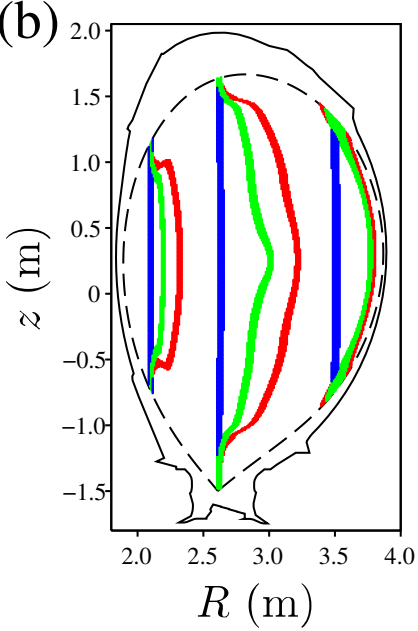

(c)

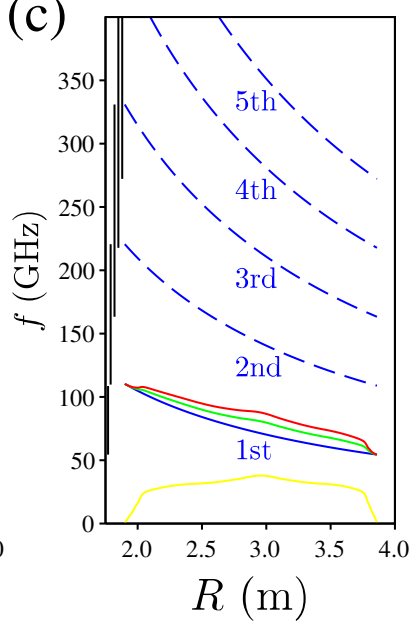

Figure 2. Standard equilibrium quantities and wave features dependent on major radius $R$ and height $z$ for JET pulse 92436 at time $t=1.9981 \mathrm{~s}$ during Ohmic phase. (a) Magnitude of magnetic field strength $|\vec{B}|$ (colour-coded) and flux surface geometry (closed white lines for normalised flux surface values $\psi_{N}=0.001,0.05,0.2,0.4,0.6$, and 0.8 ) for poloidal cross-section of JET. The first wall (solid black) of JET and the last closed flux surface (dashed-black) are shown. (b) Positions of electron cyclotron resonance (blue), upper-hybrid resonance (green), and right-hand cut-off (red) for frequencies $f=60,80$ and $100 \mathrm{GHz}$. Positions have been determined with "cold" plasma approximations, and the electron density profile used was derived with the standard approach for the reflectometer RX. (c) Quantities like in (b) along $R$ at fixed $z=0.353 \mathrm{~m}$ (height of antenna for diagnostic MIX). The plasma frequency (yellow), some harmonics of the electron cyclotron frequency (dashed-blue), and harmonic domains (solid-black) have been added. An overlap is missing only for the first and second harmonic ranges.

at the magnetic axis $(M A)$ and at the last closed flux surface $(L C F S)$, respectively. Hence, $\psi_{N}$ vanishes at the plasma centre and becomes unity at the last closed flux surface.

\section{Toroidal Magnetic Field and Its Correction}

Two different models are used to evaluate the toroidal magnetic field component $B_{\Phi}$, and both models give very similar results for an Ohmic plasma. One model follows straightforwardly from the quantity $f_{\theta}\left(\psi_{N}\right)$, including a contribution from the vacuum toroidal magnetic field $B_{\Phi V a c}$. The other model takes $B_{\Phi V a c}$ at $R_{0}=2.96 \mathrm{~m}$ and employs the familiar expression $B_{\Phi}(R, z)=B_{\Phi V a c} R_{0} / R$.

The toroidal component is usually much larger than the poloidal contribution. Hence, the magnitude of the magnetic field strength $|\vec{B}|=\sqrt{B_{\Phi}^{2}+B_{\theta}^{2}}$ is constant along almost vertical chords depicted in figure $2(\mathrm{a}) . B_{\Phi}$ is determined mainly by $B_{\Phi V a c}$, and the standard way at JET derives $B_{\Phi V a c}$ from the currents flowing inside the toroidal field coils. However, the standard technique to measure this current has a systematic error, such that $B_{\Phi V a c}$ suffers an underestimation ${ }^{32}$. This is modelled by the free parameter $b_{\Phi}$ in terms of percentage, and a scaled contribution $\left(1+b_{\Phi} / 100\right) B_{\Phi V a c}$ follows.

The prior probability $p\left(b_{\Phi}\right)$ is chosen to be uniform. The limits $0 \%$ and $5 \%$ of the corresponding interval are set to enforce an increase in $B_{\Phi V a c}$.

\section{Electron Kinetic Profiles}

The plasma electron species is captured by the temperature profile $T_{e}\left(\psi_{N}\right)$ and the density profile $n_{e}\left(\psi_{N}\right)$, implying that each quantity, and, thus, the electron pressure is preserved on a flux surface.

Since both profiles are continuous functions, they are modelled as Gaussian processes ${ }^{33}$, being independent one each other though. This means that the joint prior for the chosen parameterisation, representing each profile at distinct flux nodes, is given by a multi-variate normal distribution. The prior mean values can be set to small values, and prior covariances are selected according to model assumptions. For example, a squared-exponential ${ }^{33}$ prior covariance allows for a finite correlation over a certain length-scale between profile parameters. Since profile domains of the plasma core and edge can have significantly different length-scales, a generalised squared-exponential covariance was derived for a multi-length-scale application (see Appendix A). Other covariances would be appropriate as well, for instance to estimate the hyperbolic tangent shape of the pedestal ${ }^{21}$.

Any additional parameter associated with the prior covariance must be inferred. Thereby, related prior distributions are chosen here to be uniform, covering each a reasonable parameter domain. For examples, a lengthscale in terms of $\psi_{N}$ should be larger than zero and smaller than unity. 


\section{ECE SPECTRA AND DIAGNOSTICS MODELS}

The theory of electron cyclotron emission is well understood given a magnetised plasma with slowly varying parameters. As a consequence, detailed models, so-called ECE ray-tracing models like SPECE, have been derived to predict broadband ECE spectra. However, the complexity of multi-reflections at the three dimensional inner wall of a fusion machine is computationally disadvantageous, and, thus, approximations need to be made. The remaining detailedness included in ray-tracing models has a computational demand which is still not suitable to interpret measured data when a probabilistic approach is used.

The model ECEPT with reduced complexity was derived which remains accurate enough for the standard observation of a tenuous plasma, at least. This model lends itself to the computational demands of probabilistic inference. Furthermore, the model uses a multi-reflection approach which considers the LFS and HFS side walls to have distinct properties. A useful outcome from this approach is an experimental test for the wall properties when a low density plasma is observed.

Experimentally, ECE spectra can be accessed with different diagnostic principles. For JET, the models for two Martin-Puplett interferometers and a heterodyne radiometer model are developed, taking into account basic diagnostic features.

\section{A. ECE Spectra: Fundamentals}

The thorough theoretical deduction for the physics of electron cyclotron emission can be found in Ref. ${ }^{34}$. Essential aspects and common approaches in the field are briefly presented.

A fundamental assumption often made is that geometrical optics is valid. This implies that diffraction effects can be neglected, and any changes in the plasma parameters are considered to happen on larger temporal and spatial scales than the inverse of the wave frequency $f$ and the associated vacuum wavelength, respectively. Then, a beam used for diagnostic purposes is well approximated by a number of independent rays, and the electromagnetic wave under investigation is propagating along each ray path.

For each ray, the solution

$$
I=I_{\ell} e^{-\tau_{\ell}}+\int_{0}^{\ell} j e^{-\tau} d s
$$

of the so-called radiative transfer equation captures the intensity $I$ of the wave, having crossed a closed domain filled with an anisotropic plasma medium. The spatial integration, using the coordinate $s$, is carried out along a ray path, starting from an observer like an antenna. The integral takes into account emission, given by the coefficient $j$, and absorption, included by the coefficient $\alpha$ and its integral, i.e., the optical depth $\tau(s)=\int_{0}^{s} \alpha d s^{\prime}$, processes. The initial intensity $I_{\ell}$ undergoes absorption stated by the exponential and its argument; the optical depth $\tau_{\ell}$ for the whole ray path with length $\ell$.

Despite the simple form of equation (3), the involved quantities have complex dependencies on wave polarisation, wave vector, frequency, local magnetic field strength, distribution function parameterised by electron density and temperature to lowest order, and wall properties for the initial intensity.

\section{Ray Trajectory: Single-Pass and Multi-Pass}

A ray path in vacuum can deviate from the one taken through a plasma medium due to refraction. Hence, the determination of a ray trajectory in terms of real-space coordinates is usually the first step for an accurate model. However, the medium is surrounded by a reflecting wall, and, hence, the ray passes the plasma multiple, actually infinite, times. Thus, the accurate multi-pass trajectory includes reflections at the three dimensional inner wall. In addition, wave intensities of distinct modes, like the $\mathrm{O}$ and X-modes, become interlinked, and the multi-pass trajectory for each mode at each reflection is involved. This is not sustainable in terms of computational resources for a probabilistic approach, and, thus, approximations need to be justified and made. In the following, a distinction is adopted which splits the trajectory into a single-pass and a multi-pass contribution. For the former, $\ell$ is of the order of twice the minor radius of the fusion device, so that wall reflections are excluded. Then, the multipass ray trajectory is linked with $I_{\ell}$ and wall reflections investigated separately in Sec. IV A 5.

With the local dispersion relation for the considered wave mode at hand, the single-pass trajectory follows from the vectorial character of the group velocity for the propagating wave. Neglecting thermal effects, one finds that the trajectory depends on the vacuum line of sight of the observer, the wave mode and its cut-offs, wave frequency, plasma density, and magnetic field configuration. A lowest order approximation assigns the vacuum path to the trajectory.

\section{Resonance Condition}

To determine absorption and emission quantities along the ray trajectory, the so-called resonance condition

$$
2 \pi f=n \frac{\omega_{c}}{\gamma}+k_{\|} v_{\|}
$$

is of importance. This condition states which electrons can resonate with a wave at a given location, accounting for harmonics of the electron cyclotron frequency, a relativistic contribution to the electron cyclotron frequency 
and a Doppler term. The harmonics are counted by $n$, and the electron "cold" cyclotron frequency is given by $f_{c}=\omega_{c} /(2 \pi)=\left(e / m_{e 0}\right)|\vec{B}| /(2 \pi)$ with the elementary charge $e$, the electron rest mass $m_{e 0}$, and the magnitude of the magnetic field $|\vec{B}|$. The relativistic factor $\gamma=\left(1-|\vec{v}|^{2} / c^{2}\right)^{-1 / 2} \geq 1$ with the ratio $|\vec{v}| / c$ (magnitude of the electron velocity over speed of light in vacuum) diminishes the "cold" cyclotron frequency. The remaining term originates in the Doppler effect which gives rise to the product of the wave vector component $k_{\|}$and velocity component $v_{\|}$(both parallel to the local magnetic field direction).

If $|\vec{B}|$ varies over the plasma domain such that several distinct harmonic numbers fulfil equation (4), then several principal emission layers exist, contributing effectively to the wave intensity at a fixed frequency. Furthermore, every emission layer has a finite width due to relativistic and Doppler effects. Each of these resonance layers emits ECE radiation and absorbs emission from neighbouring resonances if present.

\section{Absorption and Emission Coefficients}

Along the ray path, absorption coefficient $\alpha$ and emission coefficient $j$ can be derived from the dielectric and current correlation tensors, respectively. These coefficients change with the wave vector, frequency, and polarisation, the distribution function of the medium, i.e., the electron species and the resonance condition.

Both coefficients are not independent on each other, because their evaluation demands the distribution function for the medium. Hence, a reformulation of the problem can be made, and the emission coefficient is replaced by the product $\alpha I_{s}$ with the intensity $I_{s}$ locally emitted.

\section{Blackbody Radiation and Temperature}

If the medium is in local thermodynamic equilibrium such that the velocity distribution is Maxwellian, then Kirchhoff law identifies the locally emitted spectrum $I_{s}$ as blackbody radiation. Furthermore, Planck law pre$\operatorname{dicts} I_{s}$ given the local temperature, i.e., $T_{e}$ for plasma electrons.

The relevant domain of the ECE spectrum is found below the frequency of $1 \mathrm{THz}$. Thus, Rayleigh-Jeans law allows a general usage of the concept of radiative temperature $T$, or brightness, instead of intensity in equation (3). For example, one finds that locally $I_{s}(f, s) \propto f^{2} T_{e}(s)$ is emitted. To be precise, the quantity $I \propto T$ located on the left-hand side of equation (3) is obtained by solving the right-hand side where the integral uses local quantities. Due to the finite width of any present emission layer, a fundamental difference exists between locally emitted blackbody radiation $\left(\propto T_{e}(s)\right)$ and experimentally accessible radiative temperature $T(f)$. However, this difference becomes small for suitable conditions like proper line of sight, high optically depth $\tau>>1$, lowtemperature and low-density plasma. These conditions imply preferred properties of the emission layer like the width approaches zero, and the layer's real-space position follows from the resonance condition.

\section{Initial Intensity and Wall Reflections}

With the distinction between single-pass and multipass ray trajectory, $I_{\ell}$ includes wall reflections and a related cross-polarisation effect, scrambling the amplitudes of X- and O-mode waves. The impact of the scrambling is insignificant on spectral domains for which the plasma is optically thick but is essential for optically thin $(\tau \simeq 1)$ ranges.

To find a precise derivation of $I_{\ell}$, the detailed wall geometry would be needed which increases the computational cost to an unacceptable extent. This common problem is usually tackled by assuming reflections between parallel walls. This allows the deduction of analytical matrix expressions from the involved quantities, like emission and absorption for each wave mode, along the single-pass trajectory. These matrices mark the limiting process of multi-reflections with the above assumptions and are applied to single-pass wave amplitudes.

\section{B. ECE Ray-Tracing Models}

The ECE ray-tracing models $\mathrm{SPECE}^{35}$ (for a $2 \mathrm{D}$ magnetic equilibrium) and TRAVIS $^{36}$ (for $2 \mathrm{D}$ and $3 \mathrm{D}$ magnetic equilibria) are two of the most accurate tools to predict broadband ECE spectra. Due to the computational demands however, the exact treatment is missing, regarding the multi-pass trajectories for different modes and the initial intensity.

Both models assume for the standard case that the plasma electrons have a Maxwellian distribution function for the velocity. In addition, non-Maxwellian and multi-Maxwellian velocity distribution functions ${ }^{37,38}$ can be considered.

For given quantities like MHD equilibrium, electron kinetic profiles, wave mode and frequency, and location and viewing angle of the observer, a single-pass ray trajectory is evaluated, considering refraction and wave cut-offs. In addition, TRAVIS can follow the multi-pass trajectory approach for an accurate three dimensional wall geometry but only for a single wave mode.

Both models evaluate the absorption coefficient from the anti-hermitian part of the fully relativistic dielectric tensor. Furthermore, blackbody emission, according to the local electron temperature, is used for Maxwellian plasmas.

SPECE estimates the initial intensity, including a cross-polarisation effect due to reflections between parallel walls. This approximative approach allows the derivation of analytic expressions for the transition from single- 
to multi-pass ECE spectra for different wave modes. The original version assigned one reflection coefficient and one polarisation-scrambling coefficient to both, HFS and LFS, walls. After having found supporting experimental evidence at JET and having derived more general analytical expressions (see Appendix B in combination with figure 4), distinct LFS and HFS properties are taken into account by SPECE.

Both models have some additional features. For instance, ECE spectra for different wave modes can be combined to match the polarisation for an antenna. Furthermore, several ray trajectories can be used to form a Gaussian beam such that an antenna pattern effect is included.

Despite the approximations already made by any ECE ray-tracing model, the computational time takes some seconds for the evaluation of one broadband spectrum. This time span is acceptable for repeating the computation for few variations of the input parameters. However, varying the parameters more than $10^{5}$ times seeks for more advanced approaches to carry out Bayesian inference. For instance, SPECE was parallelised and tested by a proof of principle ${ }^{39,40}$. The SPECE parallelisation used for this work reduces the computational time by a factor of about 10 (see Appendix C). Though, this reduction is not enough for certain operations like Markov chain Monte Carlo sampling.

\section{Input Parameters for SPECE}

For this work with SPECE, the relevant input quantities are the plasma equilibrium $\left(\psi_{N}(R, z), \psi_{M A}\right.$, $\left.\psi_{L C F S}, F_{\theta}\left(\psi_{N}\right)\right)$, the electron kinetic profiles $n_{e}\left(\psi_{N}\right)$ and $T_{e}\left(\psi_{N}\right)$, the wall reflection properties $\left(r_{L}, p_{L}, r_{H}\right.$, $\left.p_{H}\right)$, and a correction to the magnitude $b_{\Phi}$ of the vacuum toroidal magnetic field. For convenience, $F_{\theta}=\mu_{0} f_{\theta}$ is taken instead of the poloidal current function. From an accuracy point of view, the order of the Larmor radius expansion needs to be reasonably high to evaluate the dielectric tensor.

$\mathrm{X}$ - and O-mode ECE spectra are predicted at the frequency nodes provided and for the position and observation direction of an antenna. Both ECE spectra are projected to the polarisation direction accepted by the antenna. The antenna pattern can be approximated by a Gaussian beam. This beam is parameterised by the beam waist and the number of rays distributed radially and angular-wise with respect to the central ray.

\section{Tenuous Plasma Model ECEPT}

For the benefit of diminishing computational time significantly, the detailed ECE physics model is approximated by the model ECEPT, assuming a standard observation of a plasma being tenuous (PT) at lowtemperature. ECEPT predicts for given plasma param- eters broadband ECE spectra in X- and O-mode polarisations incident on a LFS antenna present for standard ECE diagnostic applications. The derived model takes into account basic cut-off and resonance features, emission and absorption, finite optical depth, reflection and polarisation scrambling at LFS and HFS walls. Further approximations applied to ECEPT would lead to a model which constitutes the basis for standard analysis techniques to derive the electron temperature profile.

The most relevant input quantities for ECEPT are the flux surface geometry, the vacuum toroidal magnetic field, the electron density and temperature profiles, reflection properties of the LFS and HFS walls, and vacuum line of sight information.

\section{Implications for Standard Observation and Tenuous Plasma}

To access the electron temperature profile by a standard ECE diagnostic application, a preferred observation of the plasma exists usually. This standard observation is characterised by a LFS antenna located at a height close to the one of the plasma centre and directed towards this centre. In addition, the optical axis of the vacuum line of sight is chosen as perpendicular as possible with respect to both, the flux surfaces (assuming a nested flux surface geometry) and the direction of the magnetic field at the plasma edge (see figure 2(a) for JET case). Related implications are captured briefly below.

For typical JET plasmas, information about the lowenergy electrons, linked to the electron temperature profile, is preserved most in the second harmonic range of the $\mathrm{X}$-mode wave (X2) and in the first harmonic range of the O-mode wave (O1). Since these modes are experimentally exploited by the observation perpendicular to the magnetic field, the focus lies on both mode types.

The wave vector of an exploited mode is almost parallel to the density gradient as the density is preserved on a given flux surface at least locally and in 0th order approximation. This minimises refraction which becomes even negligible for a tenuous (low-density) plasma. Accordingly, ray-tracing can be skipped, and the optical axis of the vacuum line of sight approximates the actual ray path.

For perpendicular observation with respect to $\vec{B}$, the Doppler effect is insignificant, and its contribution to the resonance condition is discarded.

\section{Relevant Wave Resonances and Cut-offs}

It is assumed that $\mathrm{X}$ - and $\mathrm{O}$-mode waves propagate through a weakly inhomogeneous plasma medium. For the modes under consideration and for a low-temperature plasma, basic resonance and cut-off features follow from the standard theory ${ }^{41}$. 
ECEPT presumes that inside spatial domains where the cut-off condition, depending on the mode, is fulfilled, the corresponding wave cannot propagate. When propagation towards such a domain occurs, a reflection at the cut-off layer takes place. This reflection is modelled as ideal (equal amplitudes of incident and reflected wave modes), neglecting mode conversion of any kind and tunneling across the evanescent layer.

\section{a. Electron Cyclotron Resonances}

For standard observation of the plasma, the Doppler effect can be neglected. An approximation for the relativistic effect is made by setting $\gamma=1+T_{e}\left(\psi_{N}(R, z)\right) / 511 \mathrm{keV}$ in equation (4). With the magnetic field topology at hand (see JET example $|\vec{B}(R, z)|$ in figure $2(\mathrm{a}))$, resonance locations can be found in terms of real-space coordinates dependent on $f, n$, and local electron temperature. Figure 2(c) presents these locations for $T_{e}=0$ in the poloidal cross-section of JET and along the vacuum line of sight at $z=0.353 \mathrm{~m}$ up to the fifth harmonic. For this typical case at JET, a single resonance is present in the plasma for a frequency contained in the whole first harmonic range, in most of the second harmonic range and in a small fraction of the third harmonic range. For the remaining spectral domain, the so-called harmonic overlap is evident which states that at least two resonances exist.

For completeness, figure 2(c) shows the almost vertical resonances of three frequencies from the first harmonic range inside the last closed flux surface in the poloidal cross-section.

\section{b. O-Mode Wave: Plasma Frequency Cut-off}

The cut-off condition $2 \pi f=\omega_{p}$ for the O-mode is linked to the electron plasma frequency $\omega_{p}=\sqrt{\frac{e^{2}}{m_{e 0} \epsilon_{0}} n_{e}}$, using the vacuum permittivity $\epsilon_{0}$.

At JET, this cut-off is not relevant when the density is lowered to some $10^{19} \mathrm{~m}^{-3}$ (see example in figure 2) during an Ohmic-heating phase. However, this cutoff affects the most part of the first harmonic range throughout auxiliary-heating high-density phases (see figure 17 in Ref. ${ }^{42}$ ).

c. X-Mode Wave: Upper-Hybrid Resonance, Right-Hand and Left-Hand Cut-offs

For the X-mode, the two cut-off conditions are given by $2 \pi f=\omega_{R}$ and $2 \pi f=\omega_{L}$, using the right-hand and left-hand cut-off frequencies evaluated by

$$
\omega_{L}=\frac{1}{2}\left( \pm \omega_{c}+\sqrt{\omega_{c}^{2}+4 \omega_{p}^{2}}\right)
$$

Furthermore, a resonance is present when the frequency equals the upper-hybrid frequency $\omega_{U H}=\sqrt{\omega_{c}^{2}+\omega_{p}^{2}}$.
The upper-hybrid resonance is assumed to absorb incident radiation in $\mathrm{X}$-mode polarisation completely but not to emit.

In general, the relation $\omega_{c}<\omega_{U H}<\omega_{R}$ is valid at a fixed location shown in figure 2(c). By the condition $\omega_{c}=\omega_{U H}=\omega_{R}$ (see figure $2(\mathrm{c})$ ), one finds that the upper-hybrid resonance and the right-hand cut-off are settled spatially between the electron cyclotron resonance and a LFS antenna. Hence, the right-hand cut-off and the upper-hybrid resonance are steady features for the first harmonic range, and high-density cases lead to consequences for the second harmonic range.

The layers associated with constant $\omega_{U H}$ and $\omega_{R}$ are almost vertical in the central plasma domain. Hence, this $\mathrm{X}$-mode cut-off layer acts almost like a flat mirror when a wave with matching frequency and mode is incident radially and horizontally between the heights $z=0 \mathrm{~m}$ and $0.5 \mathrm{~m}$.

The left-hand cut-off is of no interest, because wave propagation is affected only for very high density, seldomly achieved inside a JET plasma.

\section{Plasma Physics Quantities vs Common Coordinate}

With the input parameters at hand, one can connect the magnetic field amplitude, electron temperature and density along a given vacuum line of sight. Via the resonance condition, electron temperature $T_{e}\left(f_{c} / \gamma\right)$ and density $n_{e}\left(f_{c} / \gamma\right)$ become functions of the relativistically down-shifted cyclotron frequency. The locations, where the profiles vanish somewhere outside the last closed flux surface, determine the minimum and maximum values of $f_{c}$, and, thus, the fundamental and harmonic spectral ranges.

With the cut-off conditions $\omega_{p}\left(f_{c} / \gamma\right)$ and $\omega_{R}\left(f_{c} / \gamma\right)$ for both modes, and the upper-hybrid condition $\omega_{U H}\left(f_{c} / \gamma\right)$, one can determine the spatial order of features, including single or multiple cyclotron resonances, along the vacuum line of sight for one crossing through the plasma.

\section{Single-Pass ECE Spectra}

The single-pass ECE spectra for both modes leave the plasma towards LFS and HFS, respectively. To derive these spectra, the plasma electron species is assumed to obey locally the Maxwellian velocity distribution. Thus, the emissivity is replaced by the emission of a blackbody and the absorption coefficient (see Secs. IV A 3 and IV A 4). For convenience, an intensitylike quantity, like the blackbody emission, is captured in terms of radiative temperature.

For the deduction of the spectra, the spatial order of features is important, following the line of sight in vacuum inside the plasma. Each feature, being either a cut-off or a resonance, is expressed by a matrix operator, acting on the locally incident spectra. Furthermore, only 
the cyclotron resonance emits. In doing so, manageable analytical expressions can be deduced, and a basic understanding is enabled.

\section{a. Emission from Single Layer}

The resonance positions are well distinct from each other, because absorption and, thus, optical depth are vanishing away from a cyclotron resonance when the standard observation of the plasma was chosen. For each of these single emission layers, the radiative transfer equation can be solved, yielding the radiative temperature

$$
T=\frac{\int T_{e} \alpha e^{-\tau} d s}{\int \alpha e^{-\tau} d s} \int \alpha e^{-\tau} d s=\left\langle T_{e}\right\rangle\left(1-e^{-\tau}\right) .
$$

The far right-hand side of the above expression is rid from the absorption coefficient but keeps the optical depth. Furthermore, the quantity $\left\langle T_{e}\right\rangle$ marks the average for the temperature profile, using the spatial distribution of the emission layer as weighting function.

As each layer emits in both polarisations, expression (6) generalises to

$$
\begin{aligned}
\vec{T}^{n} & =\left(\begin{array}{c}
T^{X n} \\
T^{O n}
\end{array}\right) \\
& =\left(\begin{array}{cc}
1-e^{-\tau^{X n}} & 0 \\
0 & 1-e^{-\tau^{O n}}
\end{array}\right)\left(\begin{array}{c}
\left\langle T_{e}\right\rangle^{X n} \\
\left\langle T_{e}\right\rangle^{O n}
\end{array}\right)
\end{aligned}
$$

where $X$ and $O$ denote the wave modes, and $n$ labels the harmonic number.

To estimate $\left\langle T_{e}\right\rangle^{X(O) n}$, ECEPT neglects spatial distributions of any emission layer. To be precise, ECEPT assumes that changes in the physical temperature remain small inside an emission layer, and that the temperature profile itself varies on longer scales than the emission layer width. If these propositions hold, then $\left\langle T_{e}\right\rangle^{X n}(f)=\left\langle T_{e}\right\rangle^{O n}(f)=\left.T_{e}\left(f_{c} / \gamma\right)\right|_{f=n f_{c} / \gamma}$ is a good approximation, and the electron temperature is evaluated where the resonance condition is fulfilled.

When $T_{e}$ and $n_{e}$ change significantly, for example due to a large gradient, inside a resonance layer, then the emissions towards LFS and HFS can be different. Thus, a distinction between the LFS quantity $T_{L}^{X(O) n}$ and the HFS quantity $T_{H}^{X(O) n}$ needs to be made. For future purposes of ECEPT and to derive general formulas which can be used by ECE ray-tracing models, these notations are kept in the following. However, ECEPT assumes $T_{H}^{X(O) n}=T_{L}^{X(O) n}$ for the moment.

The optical depth $\tau^{X(O) n}$ is evaluated from the integral of the absorption coefficient of the corresponding emission layer for the case that the line of sight is perpendicular to the magnetic field ${ }^{43}$. This estimation takes into account wave mode and frequency, harmonic number, density and temperature, and magnetic field gradient. Density and temperature follow from the resonance condition.

\section{b. Net Emission from Multiple Layers}

If multiple emission layers exist for a fixed frequency (see $f>180 \mathrm{GHz}$ in figure 2(c)) and neither cut-off nor upper-hybrid resonance are located in-between these layers, then intensity is partly absorbed after passing through a neighbouring emission layer. The Faraday effect ensures that the polarisations of both wave modes rotate during propagation to align with the local magnetic field direction. This direction and the amplitude $|\vec{B}|$ change on spatial scales much larger than the wavelength range under consideration. Thus, it is assumed here that no cross-polarisation feature occurs, and the power associated with each polarisation direction is preserved between emission layers. Then, the attenuation by the neighbouring emission layer for the $n$-th harmonic is captured by the matrix

$$
\mathcal{T}^{n}=\left(\begin{array}{cc}
e^{-\tau^{X n}} & 0 \\
0 & e^{-\tau^{O n}}
\end{array}\right) .
$$

If a cyclotron resonance with the harmonic number $n^{\prime}$ for frequency $f$ is followed by one with the harmonic number $n$, then the net emission $\vec{T}$ towards the LFS is equated like

$$
\vec{T}=\left(\begin{array}{cc}
e^{-\tau^{X n}} & 0 \\
0 & e^{-\tau^{O n}}
\end{array}\right)\left(\begin{array}{c}
T_{L}^{X n^{\prime}} \\
T_{L}^{O n^{\prime}}
\end{array}\right)+\left(\begin{array}{c}
T_{L}^{X n} \\
T_{L}^{O n}
\end{array}\right) .
$$

For multiple subsequent emission layers, the above procedure must be applied in the right order of appearance.

\section{c. Cut-offs and Upper-Hybrid Resonance}

For the X-mode wave at a frequency of at least the first harmonic range, the important feature is the joint presence of the upper-hybrid resonance and the right-hand cut-off. For the case that the upper-hybrid resonance is approached first (propagation from HFS towards LFS antenna), the wave amplitude of the X-mode is absorbed completely, and the O-mode part passes the double feature unhindered. This filtering can be expressed by

$$
\vec{T}=\left(\begin{array}{ll}
0 & 0 \\
0 & 1
\end{array}\right) \vec{T}^{\prime}=\mathbf{F}_{O} \vec{T}^{\prime}
$$

with the matrix $\mathbf{F}_{O}$, the incident wave amplitudes $\vec{T}^{\prime}$ and the passed-through wave amplitudes $\vec{T}$. The latter quantity has a vanishing $\mathrm{X}$-mode component.

When the propagation is reversed (from LFS antenna towards HFS), the right-hand cut-off is met first. While the O-mode wave passes described by the above equation, the X-mode is reflected at this cut-off towards the lowfield side. This reflection can be written like

$$
\vec{T}=\left(\begin{array}{ll}
1 & 0 \\
0 & 0
\end{array}\right) \vec{T}^{\prime}=\mathbf{F}_{X} \vec{T}^{\prime}
$$

with the operator $\mathbf{F}_{X}$, the incident wave amplitudes $\vec{T}^{\prime}$ and the reflected wave amplitudes $\vec{T}$ with vanishing Omode component. 
Formally, an O-mode cut-off can be captured by equation (10) for the reflected part and by equation (11) for the transmitted quantity.

\section{d. LFS and HFS ECE Spectra}

ECE spectra outside the plasma domain are obtained by applying the operations for all features in proper order. In general, this results in the spectra $\vec{T}_{H(L)}$ available on the high-field $(H)$ and low-field $(L)$ sides, respectively. For example, frequencies of the first harmonic domain have one emission layer, and the upper-hybrid resonance feature is present for the LFS propagation (see figure $2(\mathrm{c}))$. Hence, one finds

$$
\vec{T}_{H}=\vec{T}_{H}^{1}=\left(\begin{array}{c}
T_{H}^{X 1} \\
T_{H}^{O 1}
\end{array}\right)
$$

and

$$
\vec{T}_{L}=\mathbf{F}_{O} \vec{T}_{L}^{1}=\mathbf{F}_{O}\left(\begin{array}{c}
T_{L}^{X 1} \\
T_{L}^{O 1}
\end{array}\right) .
$$

As one can see, the X-mode component for the LFS quantity vanishes. However, measurements at JET show that this component remains finite (see bottom of figure 4).

\section{Multi-Pass ECE Spectra}

The single-pass ECE spectra are missing essential features when compared with actual measurements. Especially, spectral domains for certain wave modes are affected for which the plasma has a small optical depth. Likewise, the vanishing single-pass X1 component of $\vec{T}_{L}$ deviates from experimental evidence. To model the missing features, a multi-pass treatment is demanded, involving reflections at the inner wall as well.

The multi-pass treatment presented considers the HFS and LFS walls to be parallel. This implies that ray trajectories for LFS and HFS propagations are transformed into each other by a wall reflection. In general, this assumption does not hold for JET (see cross-section in figure 2). Fortunately, the diagnostic vacuum lines of sight, being radial and horizontal in the height range from 0.1 to $0.4 \mathrm{~m}$, intersect with the inner wall at almost 90 degrees. Furthermore, the wall curvature for the poloidal cross-section refocusses a Gaussian beam, approximating the antenna pattern. On the contrary, the curvature for the toroidal cross-section would spread any incident Gaussian beam. To keep this effect experimentally small, the beam width at the wall should be small assumed by ECEPT.

With the assumptions made, it is possible to derive a self-repeating order of features along the trajectory. Capturing these features by matrix operators enables the analytical derivation of a multi-pass operator. This operator and the single-pass ECE spectra evaluate the multi-pass ECE spectra.

\section{a. Reflection and Polarisation-Scrambling by JET's Inner Wall}

The inner wall of JET differs in geometry and material. While the wall is made from Beryllium tiles (ITER-like wall $^{44}$ ) on the HFS side, the wall behind the in-vessel antenna array for the three ECE diagnostics is basically a port made from Inconel. Hence, the reflection properties of the HFS and LFS walls are likely to be unequal. This is modelled by assigning to the wall on the high-field (low-field) side the matrix

$$
\mathbf{W}_{H(L)}=r_{H(L)}\left(\begin{array}{cc}
1-p_{H(L)} & p_{H(L)} \\
p_{H(L)} & 1-p_{H(L)}
\end{array}\right),
$$

using in total four parameters; the reflection coefficients $r_{H(L)}$ (fraction of reflected power divided by incident power), and the polarisation-scrambling coefficients $p_{H(L)}$ (fraction to state cross-polarisation). Due to the fractional nature, a meaningful range for the coefficients is given by the interval $[0,1]$. Furthermore, all coefficients mark effective, i.e., surface-averaged quantities, and it is assumed that the coefficients have no dependency on the frequency.

\section{b. Double-Pass ECE Spectra and Operator}

For a diagnostic antenna placed on the LFS, the double-pass spectra

$$
\vec{T}_{D}=\vec{T}_{L}+\mathbf{M}_{H} \vec{T}_{H}
$$

is defined. $\vec{T}_{D}$ is incident on the antenna, if a single reflection at the HFS wall and/or cut-off layer would be present. The matrix $\mathbf{M}_{H}$ captures all ordered features such that $\mathbf{M}_{H} \vec{T}_{H}$ and $\vec{T}_{L}$ are present at the same location in front of the LFS antenna. Hence, $\mathbf{M}_{H}$ includes one reflection for each mode, attenuation by emission layers (see equation (8)) and occurring filtering due to the upper-hybrid resonance or a cut-off. The latter two features are captured by $\mathbf{F}_{X}$ and $\mathbf{F}_{O}$. If at least one wave mode reaches the HFS wall, then the wall reflection is described by $\mathbf{W}_{H}$.

The multi-pass quantities rely on the double-pass operator $\mathbf{M}_{D}$. This operator includes all present features (attenuation by plasma, cut-offs, upper-hybrid resonance) in proper order such that the product $\mathbf{M}_{D} \vec{T}_{D}$ is received together with $\vec{T}_{D}$ by the LFS antenna. Hence, $\mathbf{M}_{D}$ includes exactly two reflection for each mode. As each feature is expressed by a matrix, $\mathbf{M}_{D}$ summarises the products of matrices. In general, the first feature is the reflection at the LFS wall represented by the operator $\mathbf{W}_{L}$.

\section{c. Multi-Pass ECE Spectra and Operator}

With the double-pass quantities at hand, $\mathbf{M}_{D}^{l} \vec{T}_{D}$ marks the contribution of the $l$-th double-pass incident 


\begin{tabular}{|l|l|l|}
\hline \multicolumn{1}{|c|}{$\vec{T}_{D}$} & \multicolumn{1}{c|}{$\mathbf{M}_{D}$} \\
\hline 1 & $\mathbf{F}_{O}\left(\vec{T}_{L}^{1}+\mathcal{T}^{1} \mathbf{W}_{H} \vec{T}_{H}^{1}\right)$ & $\left(\mathbf{F}_{O} \mathcal{T}^{1} \mathbf{W}_{H} \mathcal{T}^{1} \mathbf{F}_{O}+\mathbf{F}_{X}\right) \mathbf{W}_{L}$ \\
\hline 2 & $\vec{T}_{L}^{2}+\mathcal{T}^{2} \mathbf{W}_{H} \vec{T}_{H}^{2}$ & $\mathcal{T}^{2} \mathbf{W}_{H} \mathcal{T}^{2} \mathbf{W}_{L}$ \\
\hline 2,3 & $\vec{T}_{L}^{3}+\mathcal{T}^{3} \vec{T}_{L}^{2}$ & $\mathcal{T}^{3} \mathcal{T}^{2} \mathbf{W}_{H} \mathcal{T}^{2} \mathcal{T}^{3} \mathbf{W}_{L}$ \\
& $+\mathcal{T}^{3} \mathcal{T}^{2} \mathbf{W}_{H} \vec{T}_{H}^{2}$ & \\
& $+\mathcal{T}^{3} \mathcal{T}^{2} \mathbf{W}_{H} \mathcal{T}^{2} \vec{T}_{H}^{3}$ & \\
\hline
\end{tabular}

TABLE I. Double-pass spectra $\vec{T}_{D}$ and operator $\mathbf{M}_{D}$ for lowdensity case. The quantities are listed by the number $n$ of cyclotron resonances in the plasma. Appendix B derives the lengthy expressions for the multi-pass quantities.

on the LFS antenna. Taking the limit of this repeated process equates the multi-pass ECE spectra

$$
\vec{T}_{R a d A}=\left(\sum_{l=0}^{\infty} \mathbf{M}_{D}^{l}\right) \vec{T}_{D}=\mathbf{M} \vec{T}_{D}
$$

The solution for the infinite sum

$$
\mathbf{M}=\sum_{l=0}^{\infty} \mathbf{M}_{D}^{l}=\left(\mathbf{1}-\mathbf{M}_{D}\right)^{-1}
$$

reveals the multi-pass operator with the unit matrix $\mathbf{1}$.

\section{Examples For Low Density}

The derivation of the specific mathematical expressions of $\mathbf{M}$ and $\vec{T}_{\text {RadA }}$ are quite lengthy for each spectral domain and density ranges, determining cut-offs and, thus, the proper order of features. To keep this work manageable for the reader, high density cases are left out and will be presented elsewhere. The focus lies on the lowdensity case applicable for the Ohmic-heating phase at JET. Furthermore, this case enables a basic study and an experimental test for the wall properties which are presented in Appendix B.

For the low-density case, only the right-hand cut-off needs to be considered. This case is characterised here by the conditions $\max \left(\omega_{P}\right)<\left.\omega_{c}\right|_{L F S}$ for O-mode and $\max \left(\omega_{R}\right)<\left.2 \omega_{c}\right|_{L F S}$ for X-mode. Expressions related to the low-density case remain bulky. While below the major steps for the first harmonic range are presented, the detailed derivations and results can be found in Appendix B for relevant harmonic ranges. Though, table I summarises formally the double-pass quantities from which the multi-pass entities follow.

\section{a. First Harmonic Range}

Only one cyclotron resonance exist in the first harmonic range, and the upper-hybrid resonance and the right-hand cut-off affect the $\mathrm{X}$-mode spectrum (see $60 \mathrm{GHz}<f<110 \mathrm{GHz}$ in figure $2(\mathrm{c}))$. After $\vec{T}_{H}^{1}$ is emitted by this resonance, a reflection at the HFS wall occurs.
$\mathbf{W}_{H} \vec{T}_{H}^{1}$ returns and is partially absorbed by the emission layer whilst emitting $\vec{T}_{L}^{1}$. Then, $\mathcal{T}^{1} \mathbf{W}_{H} \vec{T}_{H}^{1}+\vec{T}_{L}^{1}$ passes the upper-hybrid resonance and the right-hand cut-off layers expressed by the multiplication with $\mathbf{F}_{O}$. Altogether, this gives the double-pass ECE spectra

$$
\begin{aligned}
\vec{T}_{D} & =\mathbf{F}_{O}\left(\vec{T}_{L}^{1}+\mathcal{T}^{1} \mathbf{W}_{H} \vec{T}_{H}^{1}\right) \\
& =\left(\begin{array}{c}
0 \\
T_{L}^{O 1}+e^{-\tau^{O 1}} r_{H}\left[p_{H} T_{H}^{X 1}+\left(1-p_{H}\right) T_{H}^{O 1}\right]
\end{array}\right) .
\end{aligned}
$$

Note, the X-mode component of $\vec{T}_{D}$ has vanished.

Waves, passing by the diagnostic antenna, are reflected and polarisation-scrambled by the LFS wall written in terms of amplitude as $\mathbf{W}_{L} \vec{T}_{D}$. While the returning fraction $\mathbf{F}_{O} \mathbf{W}_{L} \vec{T}_{D}$ in O-mode polarisation enters the plasma, the waves in $\mathrm{X}$-mode polarisation remain trapped between right-hand cut-off and the LFS wall. Hence, two reflections for $\mathrm{X}$-mode are completed, and one gets $\mathbf{F}_{X} \mathbf{W}_{L} \vec{T}_{D}$

In order, the double-pass for the incident O-mode wave includes absorption by emission layer, reflection at HFS wall (cross-polarisation occurs due to polarisationscrambling), absorption by emission layer, and passing upper-hybrid resonance and right-hand cut-off region. Carrying out the associated operations yields the doublepass operator

$$
\begin{aligned}
& \mathbf{M}_{D}=\left(\mathbf{F}_{O} \mathcal{T}^{1} \mathbf{W}_{H} \mathcal{T}^{1} \mathbf{F}_{O}+\mathbf{F}_{X}\right) \mathbf{W}_{L}= \\
& \left(\begin{array}{cc}
r_{L}\left(1-p_{L}\right) & r_{L} p_{L} \\
\frac{r_{L} r_{H}}{e^{2 \tau^{O 1}}}\left(1-p_{H}\right) p_{L} & \frac{r_{L} r_{H}}{e^{2 \tau} O 1}\left(1-p_{H}\right)\left(1-p_{L}\right)
\end{array}\right) .
\end{aligned}
$$

With equation (17), the multi-pass limit gives

$$
\begin{aligned}
& \mathbf{M}= \\
& \frac{1}{D_{1}}\left(\begin{array}{cc}
1-\frac{r_{L} r_{H}}{e^{2 \tau} \tau_{1}}\left(1-p_{H}\right)\left(1-p_{L}\right) & r_{L} p_{L} \\
\frac{r_{L} r_{H}}{e^{2 \tau O 1}}\left(1-p_{H}\right) p_{L} & 1-r_{L}\left(1-p_{L}\right)
\end{array}\right)
\end{aligned}
$$

with the determinant $D_{1}=\left|\mathbf{1}-\mathbf{M}_{D}\right|$ (see expression (B6)). With equation (18), the multi-pass spectra

$$
\begin{aligned}
\vec{T}_{\text {RadA }}= & \frac{T_{L}^{O 1}+e^{-\tau^{O 1}} r_{H}\left[p_{H} T_{H}^{X 1}+\left(1-p_{H}\right) T_{H}^{O 1}\right]}{D_{1}} \\
& \times\left(\begin{array}{c}
r_{L} p_{L} \\
1-r_{L}\left(1-p_{L}\right)
\end{array}\right)
\end{aligned}
$$

follows which is incident on the diagnostic antenna. Indeed, finite values of $r_{L}$ and $p_{L}$ enhance the X-mode component. Furthermore, a very important results is found; the ratio of both components depends on the wall properties only. Thus, if both polarisations are probed, then properties of the LFS wall are experimentally accessible.

\section{Spectra Coupled to Detection System}

Usually, the $\mathrm{X}$ - and O-mode polarisation directions mismatch with the fixed orientation of an receiving 
antenna of a detection system. Hence, the detection of pure $\mathrm{X}$ - or O-mode components is seldomly possible but a linear combination of both. This effect can be included by estimating the mismatch in angular terms. Then, the finite coupling of both wave modes to the detection system is captured by Malus' law and its operator, acting on $\vec{T}_{\text {RadA }}$. Furthermore, a present or absent selecting polariser close to the antenna has an impact and needs to be addressed.

\section{a. Mismatch at JET and $W 7-X$}

By the Faraday effect, the $\mathrm{X}$ - and O-mode polarisation directions align with the local magnetic field inside the plasma. The local magnetic field, i.e., the magnetic confinement differs fundamentally for a tokamak like JET and a stellarator like W7-X. Hence, the mismatches of both machines have different characteristics.

For JET, the plasma current $I_{P}$ is ramped in time usually to achieve the magnetic confinement. Since $I_{P}$ influences the poloidal component $B_{\varphi}$, both quantities change significantly throughout a JET pulse (see figure 3). On the contrary, the toroidal magnetic field remains often constant or modifies little during a JET pulse. However, a large pulse-wise variation is possible, covering the range $B_{\Phi}=1.7 \mathrm{~T}$ and $3.45 \mathrm{~T}$ at $R=2.96 \mathrm{~m}$. As a consequence, the fractions of the ECE spectra coupled to the detection system have intra- and inter-pulse dependencies. Especially, the detected signal of the first harmonic range can deviate in relative terms by $10 \%$ from the ECE spectra at the antenna (see figure $18 \mathrm{in}^{42}$ ).

W7-X uses auxiliary coils to accomplish magnetic confinement, and the small plasma current is of the order of kA. Furthermore, the magnitude of the magnetic field remains close to $2.5 \mathrm{~T}$ on axis for all discharges. Hence, the mismatch remains almost constant in time.

\section{b. Model for Mismatch}

In angular terms, the mismatch between $\mathrm{X}$ and O-mode polarisation directions and an horizontal antenna is estimated by the pitch angle $\beta=\arcsin \left(B_{\varphi} / \sqrt{B_{\varphi}^{2}+B_{\Phi}^{2}}\right)$ at the intersection of the last closed flux surface $\left(\psi_{N}=1\right)$ with the vacuum diagnostic line of sight specific to each diagnostic. Typically for JET, $\beta$ rises to values between $10^{\circ}$ and $20^{\circ}$ shown in figure 3 .

With the pitch angle, the projection matrix

$$
\mathbf{M}_{\beta}=\left(\begin{array}{ll}
\cos ^{2} \beta & \sin ^{2} \beta \\
\sin ^{2} \beta & \cos ^{2} \beta
\end{array}\right)
$$

is evaluated, according to Malus' law. Then, the quantity $\mathbf{M}_{\beta} \vec{T}_{\text {RadA }}$ is aligned with the antenna orientation.

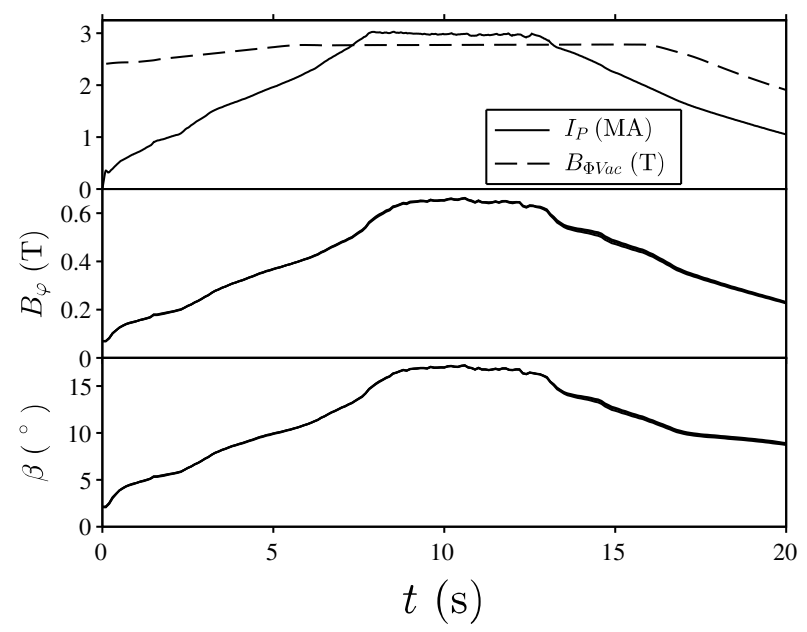

Figure 3. Plasma current $I_{P}$, toroidal vacuum magnetic field $B_{\Phi V a c}$, poloidal magnetic field $B_{\varphi}$ at last-closed flux surface intersected by vacuum ECE diagnostic (MIX, MIO, HR) lines of sight and corresponding pitch angle $\beta$ (of the magnetic field line) for JET pulse 92436 . As $B_{\Phi V a c}$ varies little during the pulse, $B_{\varphi}$ and $\beta$ follow $I_{P}$. Since the vacuum line of sights of the three diagnostics are located centrally with respect to the plasma column, the three traces shown for $B_{\varphi}$ and $\beta$ are almost identical.

The spectrum to be probed depends on the orientation and location of the selective polariser. When this orientation is not aligned with the antenna orientation, another rotation matrix needs to be applied. Furthermore, if the location of the polariser is far away from the antenna, the transmission line might cause polarisation conversion.

For the interferometers MIX and MIO (see Sec. IV D 1), a polariser is located at the end of each antenna just outside the JET vessel. In addition, the orientations of antenna and associated polariser match, so that mainly $\mathrm{X}$ - and O-mode is probed, respectively. For this case, the single spectrum

$$
T_{R a d}=\vec{F}_{P o l}^{T} \mathbf{M}_{\beta} \vec{T}_{R a d A}
$$

is coupled to the transmission line, and $\vec{F}_{\text {Pol }}$ marks the polarisation direction selected. For a diagnostic, probing mainly X-mode polarisation, $\vec{F}_{P o l}^{T}=(1,0)$ follows, and for a mainly O-mode application, one sets $\vec{F}_{P o l}^{T}=(0,1)$.

The heterodyne radiometer at JET selects the polarisation at the end of the transmission line with the length of about $45 \mathrm{~m}$. The possible polarisation conversion is interpreted as a diagnostic effect and is dealt with in Sec. IV D 2. For this diagnostic approach, spectra of both polarisations enter the transmission line like

\section{c. Selective Polariser}

$$
\vec{T}_{\text {Rad }}=\mathbf{M}_{\beta} \vec{T}_{\text {RadA }} .
$$




\section{Limitations of ECEPT}

The velocity distribution of the plasma electrons is assumed to be Maxwellian. A generalisation to arbitrary but meaningful velocity distributions is not expected to be achievable.

Refraction and the derivation of ray trajectories for given plasma parameters are not included to keep the computational time small. It is not envisaged to include these features in future works, since the corresponding slowing down of the computational time would conflict with the purpose of ECEPT.

The accurate relativistic and Doppler contributions are neglected in the resonance condition. Hence, the finite width of the emission layer is not taken into account. However, this could be implemented in future works by approximating the layer shape by a Gaussian, depending on wave properties and plasma parameters, at least. Alternatively, the local absorption coefficient could be taken from Ref. ${ }^{43}$ to account for the emission layer.

The finite size of the antenna pattern is neglected, and only the optical axis of the vacuum line of sight is considered. It seems possible to expand ECEPT to allow for several rays forming a Gaussian beam with reasonable beam waist.

\section{Diagnostic Models}

The ECE diagnostic system at JET consists of two absolutely calibrated Martin-Puplett interferometers ${ }^{42,46}$ (MIX and MIO) and the heterdyne radiometer ${ }^{47} \mathrm{HR}$. The diagnostic antennae are located on the low field side, and the radial and horizontal vacuum lines of sight pass close to the central plasma domain at the heights $0.353 \mathrm{~m}$ (MIX), $0.248 \mathrm{~m}$ (HR), and $0.133 \mathrm{~m}$ (MIO) (see figure 2(a)).

Below, the models and the likelihoods are presented for the aforementioned diagnostics. Thereby, the model for HR arrives to the actual measured data. The two interferometer models predict effective data in terms of radiative temperature to reduce complexity and save computational time. Furthermore, effective data, relying on measurements, and their estimated uncertainties are presented for the JET pulse 92436 around the time $1.9981 \mathrm{~s}$.

\section{Martin-Puplett Interferometers}

For each interferometer, the radiative temperature $T_{\text {Rad }}(f)$ stated by equation (23) undergoes the measuring principle. More precise, the product $T_{R a d} S_{T}$ with the diagnostic sensitivity $S_{T}$ is Fourier transformed to the actual data domain, detected in terms of Volt, amplified and sampled. Additionally, diagnostic imperfections are inevitable. As a consequence, the extensive model for each interferometer, stated by equation (11) in Ref. ${ }^{42}$, would need to estimate the zero-path difference location, a slowly drifting voltage offset, and the asymmetry in the interferometric data set as well. To some extend, this was investigated in Ref. ${ }^{48}$, but the computational effort for the extensive model would be enormous to gain additional diagnostic information.

Instead, a simplified model is considered which predicts the effective data in terms of radiative temperature obtained with the standard analysis ${ }^{42}$ from the actual measurements. This effective data carries an inherent convolution which is essential for this diagnostic type and must be included. The diagnostic imperfections have been investigated ${ }^{42}$, and their uncertainties are propagated to the uncertainty in the effective data.

\section{a. Convolution Model}

The spectral shape of the convolution function is determined by two features. On one side, this diagnostic type achieves only a finite maximal optical path difference, implying that the number of probed Fourier coefficients remains finite. On the other side, any standard analysis procedure applies normally an additional spatial window function to the measured interferometric data set, modifying the convolution function. Incorporating the convolution operation leads to the spectral integral

$$
T_{\text {Rad }}\left(f^{\prime}\right)=\int T_{\text {Rad }}(f) \operatorname{Conv}_{N}\left(f^{\prime}-f\right) d f
$$

where the normalised convolution $\operatorname{Conv}_{N}\left(f^{\prime}-f\right)$ has been used. Thereby, the radiative temperature $T_{R a d}(f)$ in the integral kernel marks the quantity coupled to the transmission line stated by equation (23).

For the JET case, $\operatorname{Conv}_{N}\left(f^{\prime}-f\right)$ is derived analytically in Appendix D, is shown in the top of figure 4, and has the full width at half maximum (FWHM) of about $9 \mathrm{GHz}$. Thus, features present on smaller spectral scales are filtered out by the convolution operation which can be seen in the middle of figure 4 for a box car test function $T_{\text {Rad }}(f)$. The same picture presents $T_{\text {Rad }}\left(f^{\prime}\right) \mid$ which is the convoluted quantity evaluated at the frequency nodes (principal frequencies) of the effective data of MIX and MIO.

For given principal frequencies $f^{\prime}$, the convolution integral is performed numerically for an equidistant spectral grid with a selectable increment, usually well below $1 \mathrm{GHz}$. To keep the systematic error small for this operation, a prediction $T_{R a d}(f)$, made at a finite number of spectral nodes, is interpolated to a finer spectral grid. This allows the user to remain in control and in charge of the numerics, instead of hiding it.

The convoluted quantity predicts the effective data

$$
T_{R a d}^{M I X}=T_{R a d}^{M I X} \mid+\mathcal{N}\left(0,\left(\sigma_{R a d}^{M I X}\right)^{2}\right)
$$

for MIX at the principal frequencies, assuming a normal distribution to capture the uncertainty. 

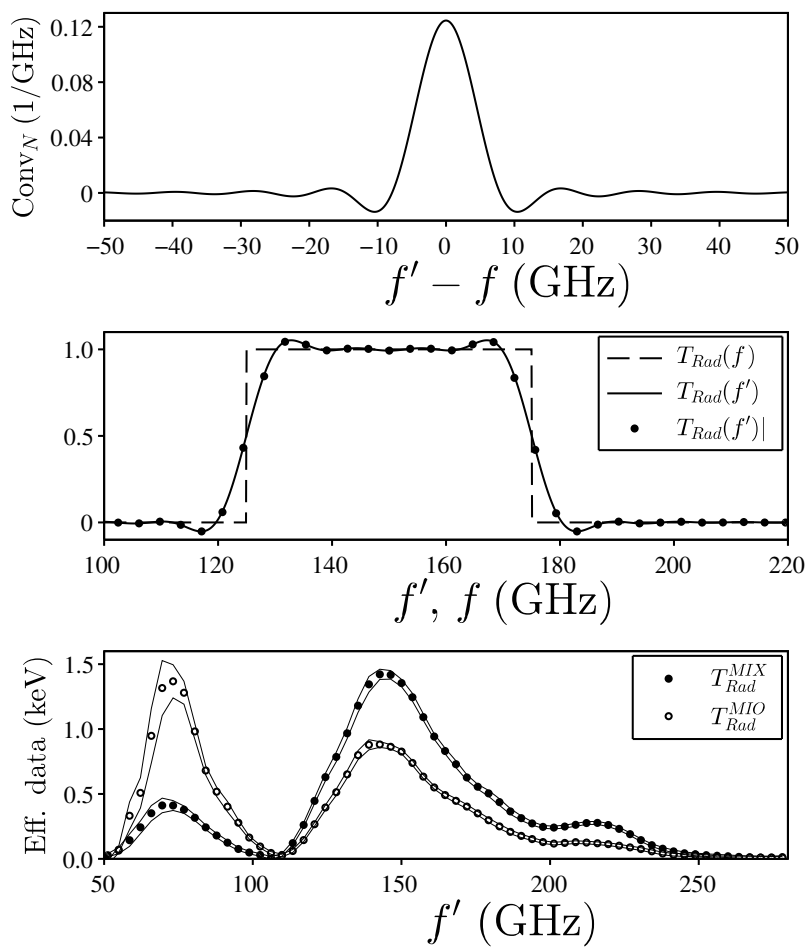

Figure 4. Convolution function and effective data for interferometers MIX and MIO. The frequencies $f$ and $f^{\prime}$ label the spectral coordinate before and after the convolution operation. Top: Normalised convolution function $\operatorname{Conv}_{N}\left(f^{\prime}-f\right)$. The full width at half maximum (FWHM) reads about $9 \mathrm{GHz}$. Middle: Comparison of box car test function $T_{\text {Rad }}(f)$ (dashed) with its convolution $T_{\text {Rad }}\left(f^{\prime}\right)$ (solid). The test function is finite and constant for the spectral domain from $125 \mathrm{GHz}$ to $175 \mathrm{GHz}$. The convoluted quantity deviates severely from $T_{R a d}(f)$ in regions where the original varies on a smaller scale than the FWHM of $\operatorname{Conv}_{N}\left(f^{\prime}-f\right)$. $T_{\text {Rad }}\left(f^{\prime}\right) \mid$ (dots) follows at the principal frequencies of the effective data. Bottom: Effective data $T_{\text {Rad }}^{M I X}$ (dots) and $T_{R a d}^{M I O}$ (circles) determined with standard analysis at JET. The 1- $\sigma$ uncertainty band (solid) is estimated via Gaussian error propagation of uncertain quantities in the standard analysis (a constant term of $10 \mathrm{eV}$ ) and of the long-term change in the absolute sensitivity.

\section{b. Effective Data and Likelihood}

After the interferometric data sets are inverse Fourier transformed and a division by the diagnostic sensitivity $S_{T}$ (or calibration) has been carried out, the effective data sets $T_{R a d}^{M I X}\left(f^{\prime}\right)$ and $T_{R a d}^{M I O}\left(f^{\prime}\right)$ are obtained, respectively. These effective data sets must be interpreted as temporal averages, according to the common diagnostic temporal resolution of $16.7 \mathrm{~ms}$. These spectra are available at principal frequencies, forming an equidistant spectral grid with the increment of about $3.66 \mathrm{GHz}$. The examples of $T_{R a d}^{M I X}\left(f^{\prime}\right)$ and $T_{R a d}^{M I O}\left(f^{\prime}\right)$ shown in the bottom of figure 4 cover the range $51.23-278.13 \mathrm{GHz}$ and have finite amplitudes up to the third harmonic range, at least.

The uncertainties on $T_{R a d}^{M I X}\left(f^{\prime}\right)$ and $T_{R a d}^{M I O}\left(f^{\prime}\right)$ are estimated with Gaussian error propagation, taking into account the noise and the diagnostic absolute calibration which contributes by far the most. The measurement noise settles at the small level of $10 \mathrm{eV}$ and enters additively which keeps the uncertainty finite for vanishing radiative temperature. The uncertainty of the calibration used here omits a possible antenna patter effect but includes several aspects like long-term variations ${ }^{42}$. When the uncertainty on the sensitivity is propagated to the effective data, a relative uncertainty $\sigma_{R e l}^{M I X(M I O)}$ follows due to the multiplication with the inverse sensitivity. Altogether, the absolute uncertainty

$$
\sigma_{\text {Rad }}^{M I X}=\left[(10 \mathrm{eV})^{2}+\left(\sigma_{\text {Rel }}^{M I X} T_{\text {Rad }}^{M I X}\right)^{2}\right]^{1 / 2}
$$

follows for MIX, and similarly for MIO, and rises with the effective data. This can be seen especially for the first harmonic ranges probed with both diagnostics (see bottom of figure 4), because the sensitivity is more uncertain.

Finally, the Gaussian likelihoods take the form

$$
\mathcal{L}^{M I X(O)} \propto \exp \left[-\frac{1}{2} \sum_{f^{\prime}} \frac{\left(T_{R a d}^{M I X(O)}-T_{R a d}^{M I X(O)} \mid\right)^{2}}{\left(\sigma_{\text {Rad }}^{M I X(O)}\right)^{2}}\right] .
$$

\section{Heterodyne Radiometer}

With the heterodyne technique, a spectral domain is down-converted from the radio frequency domain (at least some tens of $\mathrm{GHz}$ ) to the intermediate frequency domain usually below $20 \mathrm{GHz}$. This down-conversion is achieved by mixing the signal from the plasma, attenuated by the transmission line, with the signal of a local oscillator at frequency $f_{L O}$. In the intermediate spectral domain, narrow spectral bands are cut out by additional filters with bandwidths well below $1 \mathrm{GHz}$, and each of these bands is detected by a Schottky diode subsequently. As a consequence, this measurement technique allows to probe spectra emitted by plasma electrons at a much finer spectral grid when compared to the one achieved by an interferometer like MIX. Furthermore, a variable temporal resolution up to some $\mathrm{MHz}$ can be achieved.

At JET, the heterodyne radiometer ${ }^{47}$ is operated with up to 96 spectral channels. These channels can be placed in the radio frequency domain which corresponds to the first harmonic range, detecting mainly O-mode contributions, and to the second harmonic range, probing the plasma emission mainly in X-mode polarisation. Thereby, the placement of the spectral channels depends on the magnetic field strength, varying pulse-wise. This flexibility demands a pulse-wise absolute calibration, the so-called cross-calibration, of the radiometer channels which relies on the absolutely calibrated interferometers MIX and MIO. 


\section{a. Linear Model in General Form}

The diagnostic model for the radiometer presented below lacks the down-conversion. Instead, the intermediate frequency $f_{I F}$ of a given radiometer channel is converted to its radio frequency counter part by the sum $f_{H R}=f_{I F}+f_{L O}$. Thereby, it is assumed that $f_{L O}$ is constant in time. Then, each spectral channel is centred at the frequency $f_{H R}$ and has a bandwidth of $0.25 \mathrm{GHz}$. This small bandwidth is omitted in the model, and all quantities are evaluated only at $f_{H R}$. The compact diagnostic model, assuming a linear response for all channels, is written like

$$
\begin{aligned}
\left(\begin{array}{c}
V_{H R}^{X}\left(f_{H R}, t\right) \\
V_{H R}^{O}\left(f_{H R}, t\right)
\end{array}\right) & =\mathbf{A} \cdot \mathbf{S} \cdot \vec{T}_{R a d}^{H R} \mid \\
& +\left(\begin{array}{c}
V_{O f f}^{X} \\
V_{O f f}^{O}
\end{array}\right)+\left(\begin{array}{c}
\mathcal{N}\left(0,\left(\sigma_{H R}^{X}\right)^{2}\right) \\
\mathcal{N}\left(0,\left(\sigma_{H R}^{O}\right)^{2}\right)
\end{array}\right) .
\end{aligned}
$$

where $V_{H R}^{X}\left(f_{H R}, t\right)$ and $V_{H R}^{O}\left(f_{H R}, t\right)$ are the signals measured. Furthermore, $\vec{T}_{R a d}^{H R} \mid$ marks the contributions of the ECE spectra in $\mathrm{O}$ - and $\mathrm{X}$-mode polarisations coupled to the transmission line (see equation (24)) at the frequency $f_{H R}$.

The linear channel response factors, including for instance waveguide attenuation and detector sensitivity, and the cross-polarisation terms, which are caused by the transmission line itself, are listed in the diagonal and off-diagonal of the sensitivity matrix

$$
\mathbf{S}=\left(\begin{array}{cc}
S^{X} & S^{O X} \\
S^{X O} & S^{O}
\end{array}\right),
$$

respectively. For each channel, the matrix

$$
\mathbf{A}=\left(\begin{array}{cc}
A^{X} & 0 \\
0 & A^{O}
\end{array}\right)
$$

captures the chosen post-detection amplification, assumed to be constant and known. Two vectors take into account the voltage offsets $V_{O f f}^{X}$ and $V_{O f f}^{O}$ and the Gaussian noise contribution with a certain standard deviation. For this linear model, the free diagnostic parameters are $S^{X}, S^{O X}, S^{O}, S^{X O}, V_{O f f}^{X}, V_{O f f}^{O}, \sigma_{H R}^{X}$ and $\sigma_{H R}^{O}$, each demanding a model.

\section{b. Reduced Linear Model for X2 Channels}

The standard cross-calibration procedure at JET is examined in more detail in Appendix D. This approach assumes the vanishing of $S^{O X}$ and $S^{X O}$ (no polarisation conversion). With these simplifications, the crosscalibration seems to be reliable when radiometer channels probe the second harmonic range mainly in X-mode polarisation. From this fact, one deduces that $S^{O X}$ is small indeed. On the contrary, the standard cross-calibration fails when the first harmonic range mainly in O-mode
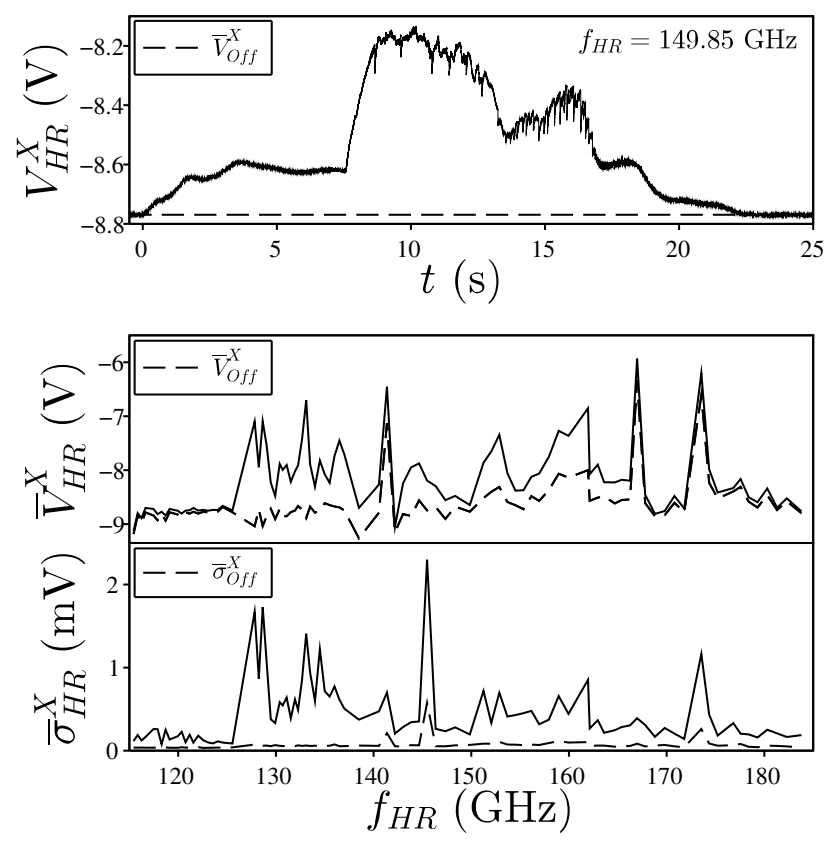

Figure 5. Signal $V_{H R}^{X}$ and effective data $\bar{V}_{H R}^{X}$ for heterodyne radiometer in X-mode configuration (JET pulse 92436). Top: $V_{H R}^{X}$ (solid) measured by channel with centre radio frequency $f_{H R}=149.85 \mathrm{GHz}$ at time $t$. The mean $\bar{V}_{O f f}^{X}$ (dashed) of the offset is estimated from data acquired before a plasma is present $(t<0 \mathrm{~s})$. Bottom: $\bar{V}_{H R}^{X}$ and associated standard deviation of mean $\bar{\sigma}_{H R}^{X}$ (both solid) for HR channels. Each signal (17 data points) has been averaged over the time window centred at $1.9981 \mathrm{~s} \pm 8.35 \mathrm{~ms}$ determined by the temporal resolution of the interferometer MIX. The offset mean and its standard deviation of mean $\bar{\sigma}_{O f f}^{X}$ (both dashed) are given.

polarisation is examined. Hence, $S^{X O}$ might be large enough to introduce a significant systematic effect. Because this aspect is still under investigation, the diagnostic model is applied only for channels which probe the second harmonic range. However, Appendix D investigates the interesting quantity $\mathbf{S}$.

With $S^{O X}=0$, one obtains the expression for the signal

$$
V_{H R}^{X}(t)=V_{H R}^{X} \mid+V_{O f f}^{X}+\mathcal{N}\left(0,\left(\sigma_{H R}^{X}\right)^{2}\right)
$$

having used the predicted plasma contribution $V_{H R}^{X} \mid=A^{X} S^{X}\left(T_{R a d A}^{H R X}\left|\cos ^{2} \beta+T_{R a d A}^{H R O}\right| \sin ^{2} \beta\right)$.

Thereby, $S^{X}, \quad V_{O f f}^{X}$ and $\sigma_{H R}^{X}$ are free diagnostic parameters for each spectral channel. For one channel, $V_{H R}^{X}(t)$ is presented in top of figure 5 .

\section{c. Offset Model}

When the plasma contribution $V_{H R}^{X} \mid$ vanishes, the offset can be estimated directly. In order to do so, data is acquired before each JET pulse at the sampling frequency of $1 \mathrm{kHz}$ for the duration of $0.5 \mathrm{~s}$. This pre-pulse data $V_{H R}^{X}(t<0 \mathrm{~s})$ is well described by the Gaussian 
$P\left(V_{O f f}^{X} \mid V_{H R}^{X}(t<0 \mathrm{~s})\right)=\mathcal{N}\left(\bar{V}_{O f f}^{X},\left(\bar{\sigma}_{O f f}^{X}\right)^{2}\right)$ with the offset mean and the standard deviation of the mean. The latter quantity can be propagated onto the uncertainty of $V_{H R}^{X}$.

The assumption that the offset remains constant during a JET pulse is valid usually (see example in top of figure 5).

\section{d. Inclusion of Uncertainty Models}

The purpose for which HR signals are used determine the uncertainty model. For example, the radiometer formula ${ }^{49}$ relates the noise of a signal with the signal itself which might allow to infer the diagnostic sensitivity from noisy data. In the following, two practical alternatives are used to estimate the uncertainty, containing measurement noise.

Each signal $V_{H R}^{X}$ is available at a much better temporal resolution than $16.7 \mathrm{~ms}$ of MIX and MIO. The variation of $V_{H R}^{X}$ from its mean $\bar{V}_{H R}^{X}$ over a small temporal window can be used to estimate the measurement uncertainty $\sigma_{H R}^{X}$. Thereby, temporal means and standard deviations should be evaluated, since the sampling frequency of the acquisition of HR signals might change during a JET pulse.

If a user is interested in the measurements at a given time, then the actual data point $V_{H_{X}}^{X}(t)$ can be used directly, and it has the uncertainty $\sigma_{H R}^{X}$. In case, a signal over a time window is of relevance, then one can approximate the data point by a Gaussian with mean $\bar{V}_{H R}^{X}$ and standard deviation of the mean $\bar{\sigma}_{H R}^{X}$. Thereby, the relation $\bar{\sigma}_{H R}^{X}<\sigma_{H R}^{X}$ holds for a finite duration. Then, one can derive the following two models, having propagated the uncertainty of the offset to the data point. For an individual point in time, one finds

$$
V_{H R}^{X}=V_{H R}^{X} \mid+\bar{V}_{O f f}^{X}+\mathcal{N}\left(0,\left(\sigma_{H R}^{X}\right)^{2}+\left(\bar{\sigma}_{O f f}^{X}\right)^{2}\right),
$$

For a temporal window, one obtains

$$
\bar{V}_{H R}^{X}=V_{H R}^{X} \mid+\bar{V}_{O f f}^{X}+\mathcal{N}\left(0,\left(\bar{\sigma}_{H R}^{X}\right)^{2}+\left(\bar{\sigma}_{O f f}^{X}\right)^{2}\right) .
$$

The latter model is the probabilistic version for the standard calibration at JET, when the window duration is given by $16.7 \mathrm{~ms}$ centred at the time when the MIX spectrum was measured.

\section{e. Effective Data and Likelihood for Calibra- tion}

For the inference part, the effective data $\bar{V}_{H R}^{X}$ and its uncertainty of 90 available channels are obtained around $t=1.9981 \mathrm{~s}$, according to the time stamp and duration of the interferometer data. $\bar{V}_{H R}^{X}$ and $\bar{\sigma}_{H R}^{X}$ are shown in the bottom of figure 5 . For valid HR channels, the estimated offset mean $\bar{V}_{O f f}^{X}$ and its uncertainty are shown in the bottom of figure 5 , and $\bar{\sigma}_{O f f}^{X}$ is smaller than $1 \mathrm{mV}$.

With the applied model (see equation (34)), the Gaussian likelihood reads

$$
\mathcal{L}^{H R} \propto \exp \left[-\frac{1}{2} \sum_{f_{H R}} \frac{\left(\bar{V}_{H R}^{X}-V_{H R}^{X} \mid-\bar{V}_{O f f}^{X}\right)^{2}}{\left(\bar{\sigma}_{H R}^{X}\right)^{2}+\left(\bar{\sigma}_{O f f}^{X}\right)^{2}}\right] .
$$

\section{RELATIVISTIC X-MODE CUT-OFF AND REFLECTOMETER MODELS}

Due to the right-hand cut-off, the X-mode wave offers the possibility to probe the electron density profile. In order to do so, a reflectometer diagnostic launches waves with varying frequency $f_{R X}^{\prime}=\omega_{R X}^{\prime} /(2 \pi)$ and with proper polarisation towards the plasma from the LFS. Waves reflected by the plasma are detected, and the frequency dependent time-of-flight or phase contain information about $n_{e}$. To extract this information, a reference measurement, i.e., the reflection at a target with wellknown position is essential. In the following, a model is derived for the standard observation which relies on the phase but predicts an optical-path-difference-like quantity to include, amongst others, straightforwardly the uncertainty of the target position of the reference measurement.

\section{A. Decision on Effective Data and Predictive Model}

Standard density profile reflectometry applications are designed to exploit the advantages of the standard observation (see Sec. IV C 1) of the plasma from the LFS. Hence, refraction is minimised, and the deviation from the radial vacuum line of sight can be neglected.

In general, the absolute phase cannot be accessed experimentally for reference and plasma measurements. Thus, phase differences $\Delta \phi_{R}\left(\omega_{R X}, \omega_{R X 0}\right)=$ $\phi_{R}\left(\omega_{R X}\right)-\phi_{R}\left(\omega_{R X 0}\right)$ (reference case without plasma) and $\Delta \phi_{P}\left(\omega_{R X}, \omega_{R X 0}\right)=\phi_{P}\left(\omega_{R X}\right)-\phi_{P}\left(\omega_{R X 0}\right)$ (plasma case after breakdown) must be evaluated to remove offsets for a suited $\omega_{R X 0}$. The difference $\Delta \phi_{P}-\Delta \phi_{R}$ cancels a remaining frequency dependent offset caused by dispersion in diagnostic hardware components like the transmission line. Writing out the terms gives

$$
\begin{aligned}
\Delta \phi_{P R}=\Delta \phi_{P}-\Delta \phi_{R} & -2 \frac{\omega_{R X}}{c}\left[\int_{R_{S}}^{R_{X C}\left(\omega_{R X}\right)} N_{X}\left(\omega_{R X}\right) d R-\int_{R_{S}}^{R_{W}} N_{V} d R\right] \\
& +2 \frac{\omega_{R X 0}}{c}\left[\int_{R_{S}}^{R_{X C}\left(\omega_{R X 0}\right)} N_{X}\left(\omega_{R X 0}\right) d R-\int_{R_{S}}^{R_{W}} N_{V} d R\right],
\end{aligned}
$$


where the signs in front of the spatial integrals determine each a positive phase change counting from the major radius $R_{S}$. Further relevant quantities are the cut-off location $R_{X C}$ and the refractive index $N_{X}$ of the wave mode and frequency under consideration, the refractive index $N_{V}=1$ for vacuum, and the location of the HFS wall $R_{W}$ where the reflection takes place for the reference measurement. Note, the major radius $R_{S}$ is distinct from the LFS boundary $R_{0}$ between plasma and vacuum domain, and the relation $R_{0}<R_{S}$ holds. Hence, $R_{S}$ locates somewhere in the vacuum domain between plasma and diagnostic antenna.

The reference integrals can be solved explicitly, and one can rewrite the above equation like

$$
\begin{aligned}
& \frac{c \Delta \phi_{P R}}{2 \omega_{R X}}+\left(1-\frac{\omega_{R X 0}}{\omega_{R X}}\right)\left(R_{S}-R_{W}\right)=\Delta R_{O p t} \\
R_{X C}\left(\omega_{R X}\right) & -\int_{R_{S}} N_{X}\left(\omega_{R X}\right) d R+\frac{\omega_{R X 0}}{\omega_{R X}} \int_{R_{S}}^{R_{X X}\left(\omega_{R X 0}\right)} N_{X}\left(\omega_{R X 0}\right) d R,
\end{aligned}
$$

and all terms have the dimension of an optical path difference. Since $N_{X}$ is less than unity in the plasma domain, $\Delta R_{\text {Opt }}$ must be of the order of the minor radius ( $\sim \mathrm{m})$ of the fusion device. Besides this less important but intuitive advantage, another one follows from data analysis point of view. Having placed $R_{S}$ somewhere in the vacuum domain by definition, the left-hand (effective data) side of equation (37) has no explicit dependency on plasma parameters, and the uncertainty on $\Delta \phi_{P R}$ and $R_{W}$ can be propagated on the effective data. Then, the predictive model follows by the numerical evaluation of the far right-hand side of equation (37).

\section{B. Relativistic Refractive Index and Cut-Off Location}

To perform a prediction, $N_{X}$ and $R_{X C}$ must be evaluated, and their relativistic expressions read

$$
N_{X}=\sqrt{1-\frac{\frac{\omega_{p}^{2}}{\gamma_{R X}}\left(1-\frac{\omega_{p}^{2}}{\gamma_{R X}} \frac{1}{\omega_{R X}^{2}}\right)}{\omega_{R X}^{2}-\frac{\omega_{p}^{2}}{\gamma_{R X}}-\frac{\omega_{c}^{2}}{\gamma_{R X}^{2}}}}
$$

and

$$
\omega_{R X}\left(R_{X C}\right)=\frac{1}{2}\left(\frac{\omega_{c}}{\gamma_{R X}}+\sqrt{\frac{\omega_{c}^{2}}{\gamma_{R X}^{2}}+4 \frac{\omega_{p}^{2}}{\gamma_{R X}}}\right)
$$

with $\gamma_{R X}=\left(1+5 T_{e} / 511 \mathrm{keV}\right)^{1 / 2}$, being valid up to at least $10 \mathrm{keV}^{50}$.

\section{Numerical Evaluation of Optical Path Difference}

$\Delta R_{O p t}$, the quantity to predict via the far righthand side of equation (37), changes with $\omega_{R X 0}$ which is chosen best by a criterion derived in the next
Section. Here, the evaluation of $R_{O p t}$ is described, and $\quad \Delta R_{O p t}=R_{O p t}\left(\omega_{R X}\right)-\left(\omega_{R X 0} / \omega_{R X}\right) R_{O p t}\left(\omega_{R X 0}\right)$ follows.

Along the major radius coordinate of the diagnostic vacuum line of sight, $T_{e}$ and $n_{e}$ profiles are mapped via the flux surface geometry, and $|\vec{B}|$ is obtained as well. Thereby, the major radius increment $\Delta R$ is a numerical parameter which is set to $1 \mathrm{~mm}$. For the corresponding spatial grid, the last vanishing electron density is found at $R_{0}$, starting from the LFS, and $R_{S}-R_{0}$ gives the vacuum contribution $\left(N_{X}=1\right)$ to $R_{O p t}$. For JET's reflectometer, $R_{S}=4.1 \mathrm{~m}$ is set to be in the vacuum domain close to the diagnostic antenna.

For the plasma domain $\left(R<R_{0}\right)$, the local quantities $\omega_{p}^{2} / \gamma_{R X}, \omega_{c} / \gamma_{R X}$ and $N_{X}$ are evaluated. $R_{X C}\left(\omega_{R X}\right)$, as obtained from equation (39) by numerical inversion, is used as a linear interpolator to retrieve for any $\omega_{R X}$ its cut-off position. It must be mentioned that for a given plasma parameter set, the part of $R_{X C}\left(\omega_{R X}\right)$ is considered as valid where it decreases monotonically, starting from the lower spectral limit. If this criterion is not met above an identified $\omega_{R X}, R_{O p t}$ is set to zero for the invalid spectral domain. Thus, this model is not able to predict a jump in $R_{O p t}$ due to, for instance, a density profile with hollow sections.

For a range large enough to cover the spectral domain of the effective data and $\omega_{R X}$ nodes separated by $0.1 \mathrm{GHz}$ (another numerical parameter), $N_{X}$ and $R_{X C}$ are computed. The numerical integration is performed from $R_{0}$ $\left(N_{X}=1\right)$ to $R_{X C}\left(N_{X}=0\right)$ for each spectral node. Thereby, $N_{X}$ is assumed to vary smoothly, such that a linear change between subsequent spatial nodes is an adequate approximation. However, this approximation is too rough for frequencies which are reflected at the plasma edge. To improve the prediction for the corresponding spectral domain, the numerical parameter $N_{R}$ is introduced which gives a lower limit on the number of spatial nodes. If $\left(R_{0}-R_{X C}\right) / \Delta R<N_{R}$, then the local quantities are re-evaluated on the spatial grid with increment $\Delta R^{\prime}=\left(R_{0}-R_{X C}\right) / N_{R}$. For example, $N_{R}$ was set to 10 , and $R_{O p t}$ is evaluated better for frequencies which get reflected inside the first $\mathrm{cm}$ of the edge domain.

With $R_{O p t}\left(\omega_{R X}\right)$ on a fine spectral grid, a linear interpolation can give the prediction $R_{O p t} \mid$ on request at an actual probing frequency $2 \pi f_{R X}^{\prime}$ of the reflectometer diagnostic.

\section{X-Mode Reflectometer}

JET's X-mode reflectometer ${ }^{45}$ (RX) consists of four back-ends which launch and detect waves at frequencies of the $\mathrm{Q}, \mathrm{V}, \mathrm{W}$, and $\mathrm{D}$ bands, respectively. By this, the range from $42 \mathrm{GHz}$ to $146 \mathrm{GHz}$ is investigated to adapt to the wide spread of possible magnetic field configurations of the tokamak and to achievable electron densities. Each back-end probes a spectral band of some tens of $\mathrm{GHz}$, and subsequent bands have an overlap of few $\mathrm{GHz}$ to 

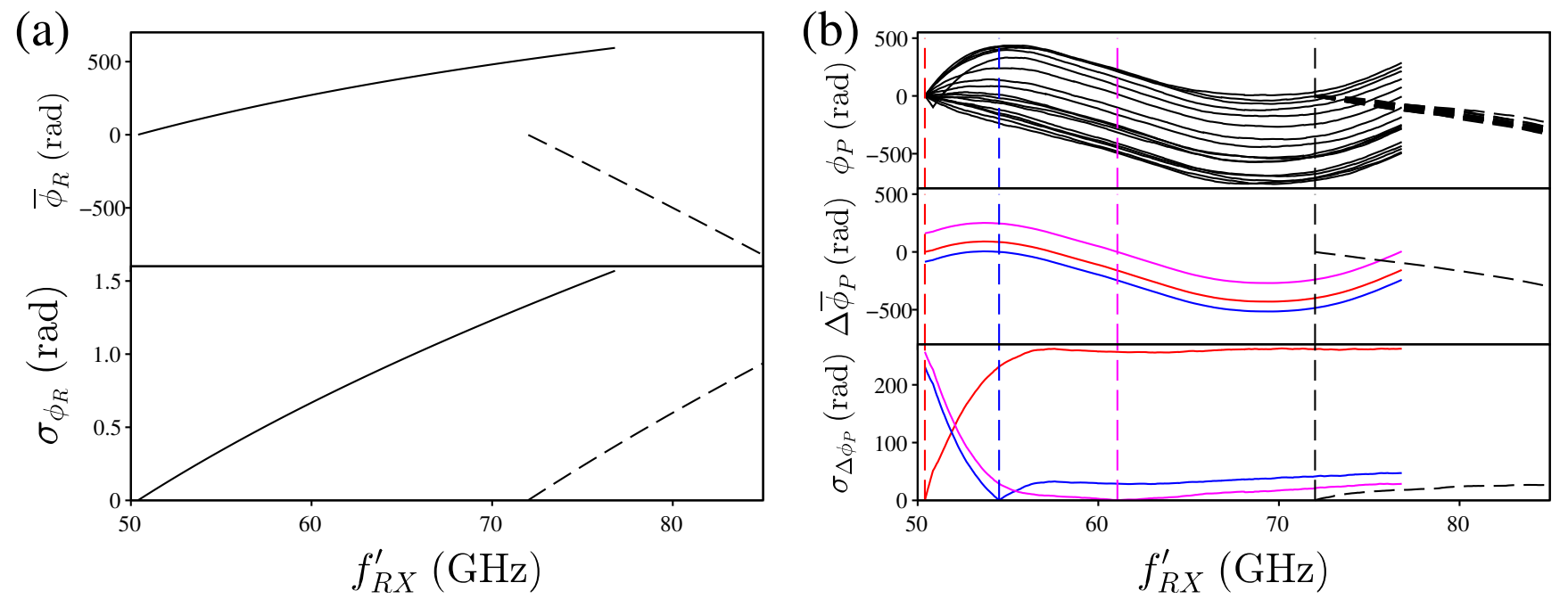

Figure 6. Phase-like quantities dependent on probing frequency $f_{R X}^{\prime}$ as determined by $\mathrm{V}$ band (solid) and W band (dashed) of X-mode reflectometer for JET pulse 92436. (a) Mean $\bar{\phi}_{R}$ and uncertainty $\sigma_{\phi_{R}}$ of reference phase. The derived quantities are obtained with a probabilistic least-square fit to the reference measurements. (b) Individual phases $\phi_{P}$ (top) acquired during temporal window $1.9981 \mathrm{~s} \pm 8.35 \mathrm{~ms}$. The temporal mean $\Delta \bar{\phi}_{P}$ (middle) and standard deviation $\sigma_{\Delta \phi_{P}}$ (bottom) of the difference phase correspond to $f_{R X 0}=50.4,54.5$ and $61 \mathrm{GHz}$ (V band, vertical coloured-dashed) and $72 \mathrm{GHz}$ (W band, vertical black-dashed). Choosing $61 \mathrm{GHz}$ reduces $\sigma_{\Delta \phi_{P}}$, such that the uncertainties of both bands are similar in amplitude.

guarantee a continuous coverage. This demerged measurement principle is captured in the following by applying a band-dependent version of equation (37). Phase data for each band are provided by the standard approach which filters the two measured voltage signals for unwanted reflection features and unwraps the phase from the remaining signals. The filtering and unwrapping are not treated by this reflectometer model.

The back-ends are connected via transmission lines to one of two launching antennae inside the JET vessel. Both antenna apertures are located at the same height of $0.258 \mathrm{~m}$ and major radius coordinate of $4.35 \mathrm{~m}$ (see figure 2(a)) but at slightly different toroidal angels. Both vacuum lines of sight remain radial. However, the two associated intersections with the HFS inner wall and its limiter have different major radii. For plasma operation, one finds the band-specific uncertain values $R_{W 1}=1.78 \mathrm{~m} \pm 0.01 \mathrm{~m}(\mathrm{~V}$ and $\mathrm{D}$ bands) and $R_{W 2}=1.816 \mathrm{~m} \pm 0.02 \mathrm{~m}(\mathrm{Q}$ and $\mathrm{W}$ bands) due to a curved limiter structure.

For the JET pulse 92436 and close to time $t=1.9981 \mathrm{~s}$, the $\mathrm{V}$ band $(50.4-76.8 \mathrm{GHz})$ and the lower spectral part of the $\mathrm{W}$ band $(72-85 \mathrm{GHz})$ are of interest. The truncation of the latter band was made, since a phase jump is evident above $85 \mathrm{GHz}$. This jump is a hint for an unfavourable electron density profile and, thus, a nonmonotonic domain in $R_{X C}\left(\omega_{R X}\right)$ which is not modelled.

\section{Reference Phase}

Before each JET pulse, the reflection at the HFS wall is used as reference measurement to compensate for any dispersion caused by diagnostic hardware. About 20 frequency sweeps are carried out, and data is collected. The routine automatic analysis applies a least-square fit to the maximum of the spectrogram of the beat frequency. A developed probabilistic version of this fit achieves a better agreement with the noisy data. The corresponding mean $\bar{\phi}_{R}$ of the reference phase and its one-sigmauncertainty $\sigma_{\phi_{R}}$ are shown in figure 6(a) for the spectral domain which is relevant for the inference.

After choosing $f_{R X 0}$ for each band (see below), the mean difference $\Delta \bar{\phi}_{R}=\bar{\phi}_{R}\left(f_{R X}^{\prime}\right)-\bar{\phi}_{R}\left(f_{R X 0}\right)$ is obtained. This difference has the variance $\sigma_{\Delta \phi_{R}}^{2}=\sigma_{\phi_{R}}^{2}\left(f_{R X}^{\prime}\right)+\sigma_{\phi_{R}}^{2}\left(f_{R X 0}\right)$ for $f_{R X}^{\prime} \neq f_{R X 0}$.

\section{Plasma Phase and Choice of $f_{R X 0}$}

The 17 phases $\phi_{P}$ obtained during the period $1.9981 \mathrm{~s} \pm 8.35 \mathrm{~ms}$ have a wide spread for the $\mathrm{V}$ band but not for the W band (see figure 6(b)). This feature originates in pure-noise contribution to the signals of the lower spectral domain for which no cut-off is present inside the plasma. Hence, a considerably uncertain phase offset follows for each $\phi_{P}$. In the domain above $55 \mathrm{GHz}$, for which a clear cut-off exists, the spectral derivatives of $\phi_{P}$, linked to the time-of-flight, seem similar, since this operation would remove the uncertain offset. The alternative, which is used here, cancels the offset present at $f_{R X 0}$ via the phase difference $\Delta \phi_{P}=\phi_{P}\left(f_{R X}^{\prime}\right)-\phi_{P}\left(f_{R X 0}\right)$.

The choice of $f_{R X 0}$ has a severe impact on the uncertainty of $\Delta \phi_{P}$. This is revealed by evaluating the mean $\Delta \bar{\phi}_{P}$ and the standard deviation $\sigma_{\Delta \phi_{P}}$ with respect to 


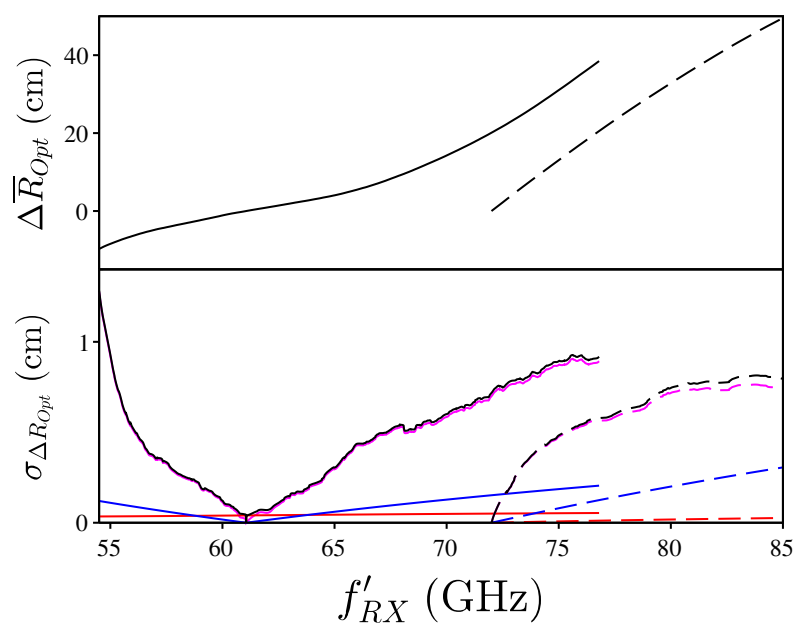

Figure 7. Top: Mean optical path difference $\Delta \bar{R}_{O p t}$ (effective data) versus probing frequency $f_{R X}^{\prime}$ for $\mathrm{V}$ (solid) and $\mathrm{W}$ (dashed) bands of X-mode reflectometer for JET pulse 92436 at time $t=1.9981 \mathrm{~s}$. Bottom: Uncertainty $\sigma_{\Delta R_{O p t}}$ on $\Delta \bar{R}_{O p t}$ for $\mathrm{V}$ (black-solid) and W (black-dashed) bands. The three contributions (coloured) to $\sigma_{\Delta R_{O p t}}$ are related to the plasma difference phase (magenta), the reference difference phase (red), and the band-dependent reference reflection location (blue) at the high-field side wall of JET.

time for different $f_{R X 0}$. For $f_{R X 0}=50.4 \mathrm{GHz}$ (lowest possible for $\mathrm{V}$ band but no reflection), $54.5 \mathrm{GHz}$ (first reflection detected) and $61 \mathrm{GHz}$ (constant time-of-flight), $\sigma_{\Delta \phi_{P}}$ remains small close to $f_{R X 0}$ but increases away from it (see bottom of figure 6(b)). However, $\sigma_{\Delta \phi_{P}}$ is reduced broadly for the $61 \mathrm{GHz}$ case, and, hence, this frequency is chosen as $f_{R X 0}$ for the $\mathrm{V}$ band. For the $\mathrm{W}$ band, the lowest frequency $\left(f_{R X 0}=72 \mathrm{GHz}\right)$ implies already a small $\sigma_{\Delta \phi_{P}}$ which is comparable with the one of the $\mathrm{V}$ band.

With the choice of $f_{R X 0}$, the mean $\Delta \bar{\phi}_{P}$ follows for each band, but the corresponding uncertainty needs to be identified. The quantity $\sigma_{\Delta \phi_{P}}$ captures noise and temporal changes due to a possible slow change in the electron density profile. Since both contributions are not further distinguished, the standard deviation $\sigma_{\Delta \phi_{P}}$ is interpreted as the uncertainty on $\Delta \bar{\phi}_{P}$ in the probabilistic scheme. This means that the standard deviation of the mean (less than $\sigma_{\Delta \phi_{P}}$ by a factor of 4 ) is of no interest, and, thus, the inference to be carried out is not overconfident.

\section{Effective Data and Likelihood}

The optical path difference, i.e., the effective reflectometer data is evaluated as

$$
\begin{aligned}
\Delta \bar{R}_{O p t}\left(f_{R X}^{\prime}\right)= & \frac{c}{4 \pi f_{R X}^{\prime}}\left(\Delta \bar{\phi}_{P}-\Delta \bar{\phi}_{R}\right) \\
& +\left(1-\frac{f_{R X 0}}{f_{R X}^{\prime}}\right)\left(R_{S}-R_{W 1(2)}\right)
\end{aligned}
$$

with the band-dependent major radius $R_{W 1}$ or $R_{W 2}$ due to the reference reflection at the HFS wall.

The noise domain below $54.5 \mathrm{GHz}$, which misses a reflection by the plasma, is excluded for the $\mathrm{V}$ band. Furthermore, the diagnostic spectral resolution (10 - $20 \mathrm{MHz})$ achieved by each band provides more than 2000 available data points. In order to reduce this number to a reasonable value below 100 , a subset of $\Delta \bar{R}_{O p t}\left(f_{R X}^{\prime}\right)$ is considered for the spectral increment of about $400 \mathrm{MHz}$. Eventually, 54 (V band) and 31 (W band) data points are at hand, excluding the vanishing $\Delta \bar{R}_{O p t}\left(f_{R X 0}\right)$ for both bands, and are presented in figure 7 .

The uncertainty $\sigma_{\Delta R_{O p t}}$ on the effective data is estimated via Gaussian error propagation as

$$
\begin{aligned}
\sigma_{\Delta R_{O p t}}^{2}= & \left(\frac{c}{4 \pi f_{R X}^{\prime}}\right)^{2}\left(\sigma_{\Delta \phi_{P}}^{2}+\sigma_{\Delta \phi_{R}}^{2}\right) \\
+ & \left(1-\frac{f_{R X 0}}{f_{R X}^{\prime}}\right)^{2} \sigma_{R_{W 1(2)}}^{2} .
\end{aligned}
$$

In the above expression, $\sigma_{R_{W 1(2)}}=1(2) \mathrm{cm}$ takes into account the uncertainty of $R_{W 1}$ and $R_{W 2}$ dependent on the band. The main contribution to $\sigma_{\Delta R_{O p t}}$ is the one from the difference of the plasma phase as shown in figure 7 .

Finally, the likelihood

$$
\mathcal{L}^{R X} \propto \exp \left[-\frac{1}{2} \sum_{V, W} \sum_{f_{R X}^{\prime}} \frac{\left(\Delta \bar{R}_{O p t}-\Delta R_{O p t} \mid\right)^{2}}{\sigma_{\Delta R_{O p t}}^{2}}\right]
$$

is formed for this diagnostic.

\section{EXAMPLE APPLICATION FOR OHMIC PLASMA}

The presented model, either using SPECE or ECEPT as predictor for ECE spectra, is tested for a low density and low temperature JET plasma (pulse 92436, at time $t=1.9981 \mathrm{~s}$ ) during the Ohmic phase with a toroidal magnetic field of $2.5 \mathrm{~T}$ and a plasma current of $1 \mathrm{MA}$. Since more than two hundred free parameters are inferred, a suitable strategy is demanded to reduce the computational duration.

\section{A. Strategy}

Given the number of free parameters and the computational time for a single prediction with SPECE, the investigation of the problem benefits from a suitable strategy. At first, the model complexity is reduced by using the less accurate plasma equilibrium model and ECEPT. With an uninformative prior, the parameter set is obtained which marks the maximum of the posterior (MAP). The numerical procedure to find this MAP is heavily prolonged, since the radiometer is included. To be more precise, the data measured with HR can be modelled well for any 
given parameter combination by adapting the radiometer sensitivity, while predictions and effective data do not match at all for the other three diagnostics. A short-cut is possible by excluding HR until a good match with the data of the remaining diagnostics is found. When the MAP is found, MCMC sampling is carried out to explore the neighbourhood, and the posterior is investigated for the reduced problem.

The above procedure to find the MAP is repeated when the accurate plasma equilibrium model and SPECE are employed. However, MCMC sampling is not feasible, and, thus, only the MAP is found which can be compared with ECEPT results.

The inference for each model does not take into account any systematic uncertainties. At the end of this section, some uncertainties are examined with respect to the usage of ECEPT and SPECE.

\begin{tabular}{|l|l|l|l|}
\hline Parameters & $\begin{array}{l}\text { Prior } \\
\text { Type }\end{array}$ & $\begin{array}{l}\text { Valid } \\
\text { Domain }\end{array}$ & $\begin{array}{l}\text { Prior Mean } \\
\text { Prior Covariance } \\
\{\text { Initial Value }\}\end{array}$ \\
\hline$T_{e}\left(\psi_{N}\right)(\mathrm{keV})$ & MVN & - & $\begin{array}{l}\psi_{N}>1: 0.02 \\
\psi_{N} \leq 1: 0.2 \\
\boldsymbol{\Sigma}_{G S E 1}\end{array}$ \\
\hline$\sigma_{T_{e}}(\mathrm{keV})$ & uniform & {$[0.01,10]$} & $\{5.0\}$ \\
\hline$l_{T_{e}}$ & uniform & {$[0.2,1]$} & $\{0.5\}$ \\
\hline$n_{e}\left(\psi_{N}\right)\left(10^{19} / \mathrm{m}^{3}\right)$ & MVN & - & $\begin{array}{l}\psi_{N}>1: 0.02 \\
\psi_{N} \leq 1: 0.2 \\
\boldsymbol{\Sigma}_{G S E 2}\end{array}$ \\
\hline$\sigma_{n_{e}}\left(10^{19} / \mathrm{m}^{3}\right)$ & uniform & {$[0.01,10]$} & $\{5.0\}$ \\
\hline$l_{n_{e} C}$ & uniform & {$[0.2,1]$} & $\{0.5\}$ \\
\hline$l_{n_{e} E}$ & uniform & {$[0.01,1]$} & $\{0.5\}$ \\
\hline$\psi_{N, n_{e}}$ & uniform & {$[0.7,1.14]$} & $\{0.7\}$ \\
\hline$r_{L}, r_{H}, p_{L}, p_{H}$ & uniform & {$[0,1]$} & $\{0.1\}$ \\
\hline$b_{\Phi}(\%)$ & uniform & {$[0,5]$} & $\{0.0\}$ \\
\hline$S^{X}\left(f_{H R}\right)(\mathrm{mV} / \mathrm{keV})$ & MVN & - & 0 \\
\hline
\end{tabular}

TABLE II. Prior choice of 217 free parameters. Electron density and temperature profiles are parameterised each by 58 parameters. For each profile parameter set, the joint prior is a multi-variate normal distribution $(\mathrm{MVN})$ with finite prior mean and covariance of type generalised-squared-exponential (see Appendix A). This kind of covariance has free parameters itself like an absolute scaling $\left(\sigma_{T_{e}}, \sigma_{n_{e}}\right)$, at least one length-scale $\left(l_{T_{e}}, l_{n_{e} C}\right.$ (core) and $l_{n_{e} E}$ (edge)) and a domain delimiter $\left(\psi_{N, n_{e}}\right)$ if needed. To these prior covariance parameters, a uniform prior is assigned over a reasonable domain likewise for the toroidal magnetic field correction $b_{\Phi}$ and the wall reflection properties $r_{L}, r_{H}, p_{L}$ and $p_{H}$. The sensitivities $S^{X}$ of 90 heterodyne radiometer channels are assumed to be independent, and the joint MVN prior is factorisable.

\section{B. Free Parameters and Assigned Priors}

The correction of the toroidal magnetic field and the wall reflection properties are accompanied by the parameters for the electron temperature and density profiles, and the sensitivity of the heterodyne radiometer. The problem is formulated, such that 217 parameters (123 plasma parameters, four wall parameters and 90 radiometer channel sensitivities) are to be estimated. These free parameters have each an uninformative prior distribution listed in table II. For the profiles, the prior distributions use covariances which depend on additional parameters like an absolute scaling and a set of length-scales.

\section{Density and Temperature Profiles}

The profiles of the electron density and temperature are modelled independently by a Gaussian process. Basically, any chosen profile parameterisation has a joint Gaussian prior (multi-variate normal distribution) with mean and covariance. For the chosen parameterisation, the electron temperature and density profile parameters locate at 58 nodes of the shared normalised flux grid with the limits 0 and 1.14 and with the increment 0.02 . Hence, profiles are assumed to vanish when $\psi_{N}$ exceeds 1.14 .

For each profile, the prior mean is set to a finite, constant and small value of $0.2 \mathrm{keV}\left(10^{19} \mathrm{~m}^{-3}\right)$ inside and $0.02 \mathrm{keV}\left(10^{19} \mathrm{~m}^{-3}\right)$ outside the last closed flux surface.

Each profile prior covariance is of the type generalisedsquared-exponential (GSE, see Appendix A) chosen to estimate length-scales of different plasma domains. For the temperature profile of an Ohmic plasma at JET, a single domain is sufficient, and, thus, the applied covariance $\Sigma_{G S E 1}$ has one length-scale parameter $l_{T_{e}}$ and the scaling parameter $\sigma_{T_{e}}$. On the contrary, the density profile has two domains even during the Ohmic phase of JET. Hence, $\Sigma_{G S E 2}$ is used with four free parameters; the scaling $\sigma_{n_{e}}$, the delimiter node $\psi_{N, n_{e}}$ between the domains, and the two length-scales $l_{n_{e} C}$ (core) and $l_{n_{e} E}$ (edge). Each of the 6 covariance parameters has a uniform prior assigned for a meaningful domain (see table II). For example, a normalised flux length-scale should be of the order of 1 for the core plasma.

\section{Magnetic Field Correction and Wall Reflection Properties}

Each of the parameters $b_{\Phi}$ (correction of the toroidal magnetic field), $r_{L}, r_{H}, p_{L}$ and $p_{H}$ (LFS and HFS wall reflection properties) has a uniform prior for the relevant domain. For $b_{\Phi}$, the prior is finite for values between $0 \%$ and $5 \%$. The wall properties can take values between 0 and 1 . 


\section{Radiometer Sensitivity}

The sensitivities for the 90 radiometer channels are assumed to be independent. To each sensitivity, a Gaussian prior is assigned with vanishing mean but large variance of $1000^{2}(\mathrm{mV} / \mathrm{keV})^{2}$.

\section{Inference Using ECEPT}

Having found the MAP, conditional posteriors for individual parameters are checked, since this the only possible way to interpret the results determined later with SPECE. At the MAP, $10^{6}$ MCMC samples are drawn from the joint posterior. The first half of the samples is rejected due to the burn-in phase of this numerical method. From the second half, the posterior mean and standard deviation are evaluated for each parameter. Furthermore, the marginal posteriors are available, and the correlation between parameters is accessible.

\section{Conditional Posteriors at MAP}

At the MAP, the conditional posterior of a single parameter can be investigated while the remaining parameters are kept constant. Usually, conditional posteriors are not of particular interest, since the joint posterior can be non-Gaussian and/or multi-modal. However, the (a)

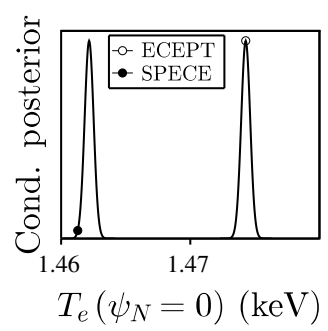

(b)

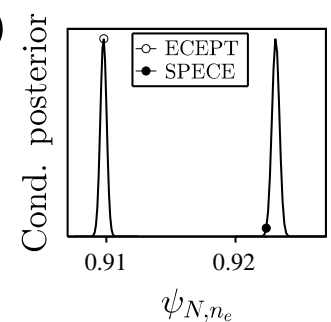

(c)

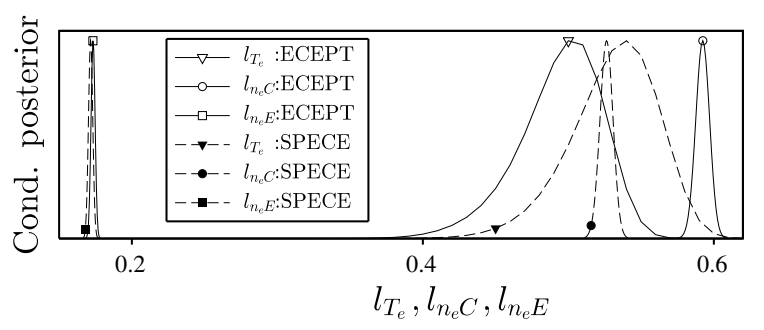

Figure 8. Examples of single parameter conditional posterior at MAP found with ECEPT (empty symbols) and SPECE (filled symbols). ECEPT and SPECE results have each a narrow shape (underestimated uncertainties) but deviate slightly. Though, these differing results are well included in the broader marginal posterior (proper uncertainties) obtained with ECEPT and shown in figure 9. (a) Central electron temperature $T_{e}$. (b) Delimiter $\psi_{N, n_{e}}$. (c) Length-scales of electron temperature and density profiles. parameter set at the MAP and the conditional posteriors are the only quantities which are currently accessible with SPECE due to computational restrictions. To compare the results of SPECE and ECEPT to some extent, the conditional posteriors have been examined, and some are presented here.

The investigation of a conditional posterior reveals a narrow distribution for an individual parameter. For example, the central electron temperature has a Gaussianlike distribution and the standard deviation of less than $1 \mathrm{eV}$ (see figure 8(a)). This holds for the central electron density as well with the standard deviation of less than $10^{16} \mathrm{~m}^{-3}$. The delimiter node $\psi_{N, n_{e}}$ peaks a bit above 0.9 with a width of about $10^{-3}$ presented in figure $8(\mathrm{~b})$. For the length-scales, the results can be found in figure $8(\mathrm{c})$.

In general, any conditional posterior is much narrower than the corresponding marginalised posterior (see below). This means that the uncertainty of a parameter is larger by about one order of magnitude after the numerical marginalisation which can be interpreted as a propagation of the uncertainty of other parameters. For instance, the marginal uncertainty of the central electron temperature reads $10 \mathrm{eV}$, and one gets $5 \times 10^{-3}$ for $\psi_{N, n_{e}}$. Usually, each marginal posterior peaks over the domain covered by the conditional one, meaning that the parameter set at the MAP is included in the marginal distributions.

\section{Marginal Posterior Distributions}

The marginal posterior for each parameter is presented in figures 9(a)-(g) and top of figure 10. For convenience, posterior samples for the profile parameters, for the wall properties and for the radiometer sensitivity are depicted together.

\section{a. Plasma Parameters}

The posterior profile samples form smooth functions, since the estimated length-scales $l_{T_{e}}, l_{n_{e} C}$ and $l_{n_{e} E}$ peak in the vicinity of 0.49 (std. dev. 0.06), 0.55 (std. dev. 0.06 ) and 0.17 (std. dev. 0.02), respectively. The absolute heights of the inferred profiles are described best by the absolute scalings close to $\sigma_{T_{e}}=2.3 \mathrm{keV}$ and $\sigma_{n_{e}}=1.6 \times 10^{19} \mathrm{~m}^{-3}$ of the prior covariance.

The mean and sample profiles approach zero above $\psi_{N}>1$. At the last-closed flux surface, one finds the uncertain values $T_{e}=(85 \pm 10) \mathrm{eV}$ and $n_{e}=(2 \pm 0.4) \times 10^{18} \mathrm{~m}^{-3}$. The core plasma profiles have uncertainties which settle at $5 \mathrm{eV}$ and $10^{17} \mathrm{~m}^{-3}$, respectively. However, the uncertainties double near the plasma centre $\left(\psi_{N}=0\right)$ missed by the diagnostic vacuum lines of sight. The uncertainties are elevated even more towards the plasma edge, especially for $n_{e}$ due to its steep gradient. The edge domain is well identified by the node $\psi_{N, n_{e}}$ with mean 0.916 and standard deviation 
(a)

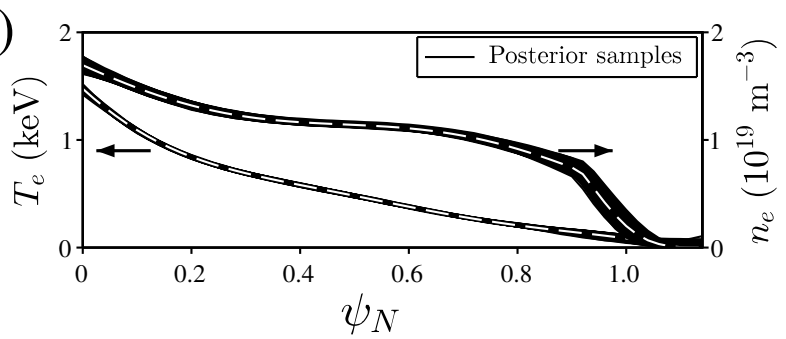

(b)

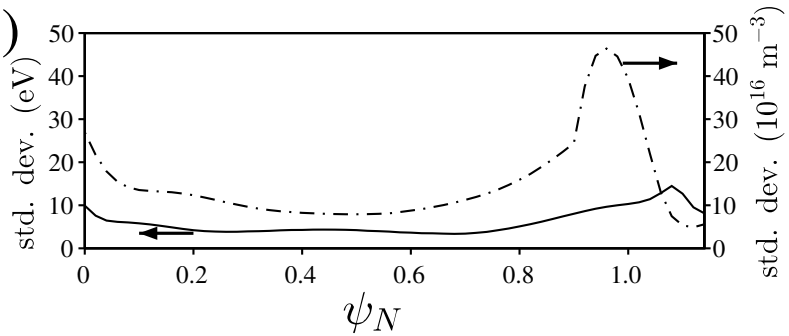

(c)

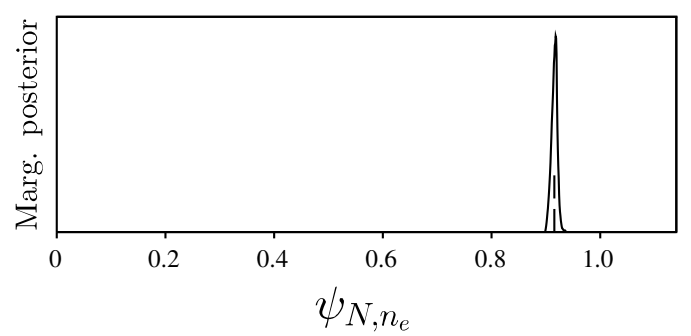

(d)

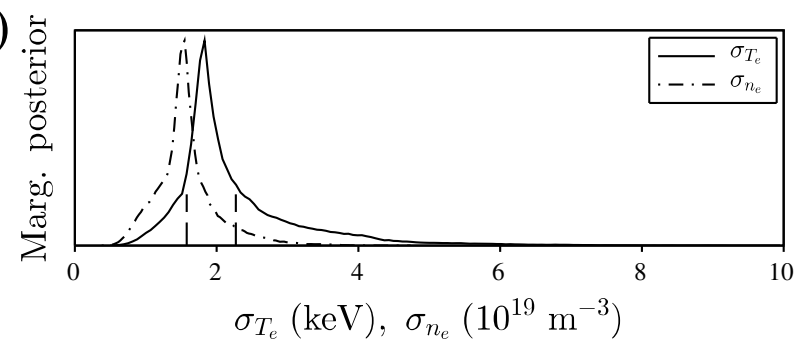

(e)

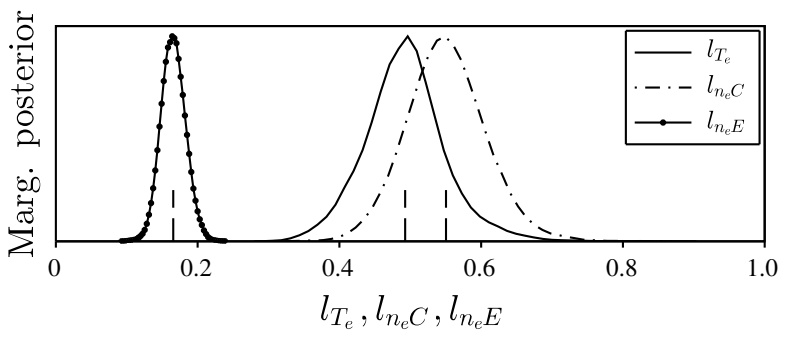

(f)

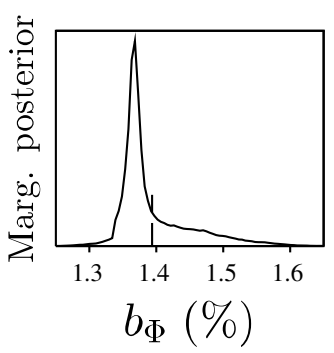

(g)

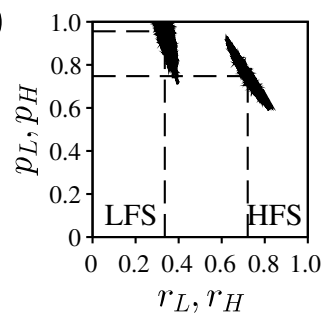

Figure 9. Marginal posterior distributions and mean values (dashed) using less accurate models for plasma equilibrium and ECE spectra (ECEPT). (a) Posterior samples (black) for electron temperature and density profiles. (b) Posterior standard deviation of electron temperature and density profiles. (c) Domain delimiter for generalised-squared-exponential prior covariance for electron density to distinguish plasma core and edge. (d) Scalings of squared-exponential prior covariance for electron density and temperature. (e) Length-scales of generalised-squared-exponential prior covariances for electron density (core and edge) and temperature. (f) Correction for toroidal magnetic field. (g) Posterior samples (black) for reflection properties of JET's inner wall located on low-field side (LFS) and high-field side (HFS).

0.005 .

The marginal posterior of the correction $b_{\Phi}$ reveals an increase of the toroidal magnetic field $(2.53 \mathrm{~T}$ at $\left.R_{M a j}=2.96 \mathrm{~m}\right)$ by about $1.39 \%$ with standard deviation $0.05 \%$.

\section{b. Wall Reflection Properties}

The samples of the LFS and HFS wall properties locate in the domains close to the mean values $\left(r_{L}, p_{L}\right)=(0.34,0.96)$ and $\left(r_{H}, p_{H}\right)=(0.72,0.75)$. Hence, the reflectivity of the ITER-like wall is about twice as high as the one of the diagnostic port. Since both walls have large polarisation-scrambling coefficients, the JET vessel itself causes a significant cross-polarisation effect. The standard deviations for all four parameters are below 0.05 .

\section{c. Radiometer Sensitivity}

The narrow band, which is obtained by presenting the posterior samples versus radio frequency, translates to small uncertainties $\sigma^{X}$ on the inferred sensitivity (see figure 10). In relative terms, the posterior mean $S^{X}$ has an uncertainty of less than $2 \%$. For channels, which probe the plasma centre, this uncertainty drops below $1 \%$.

When $S^{X}$ is compared to the mean $C^{X}$, derived and used officially at JET, a good agreement is found within the uncertainties (see bottom of figure 10). However, the inferred sensitivities deviate for the spectral domains $127.825-134.5 \mathrm{GHz}$ and $151.25-161.95 \mathrm{GHz}$. This is caused by non-linear responsivity terms left out in the corresponding diagnostic model.

\section{Posterior Correlations}

For the 217 parameters, the posterior correlations are shown in figure 11. Several significant (anti-)correlation features are found, and most of the causes seem to be 


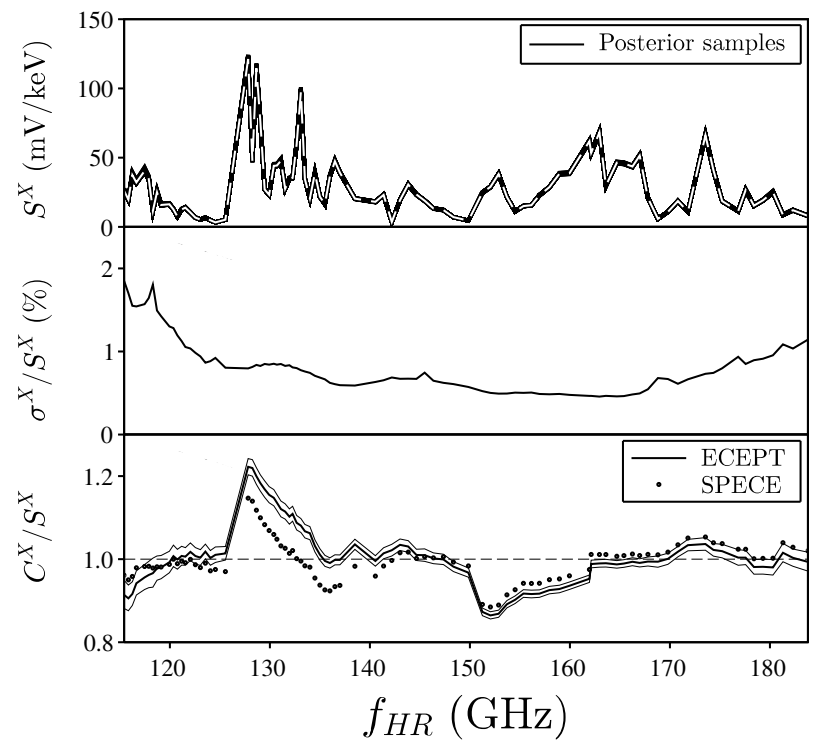

Figure 10. Marginal posterior mean $S^{X}$ and uncertainty (standard deviation $\sigma^{X}$ ) for heterodyne radiometer sensitivity. The less accurate models for plasma equilibrium and ECE spectra (ECEPT) have been applied. Top: Posterior samples (black) and $S^{X}$ (white-dashed). Middle: Relative uncertainty. Bottom: Ratio of mean sensitivities inferred by standard approach $\left(C^{X}\right)$ and Bayesian model (black-solid) shown in the top. The uncertainty on the ratio follows from $\pm 2 \sigma^{X}$. The ratio (dots) with respect to the MAP, found with the more accurate model, reveals an approach dependent uncertainty.

understood. One reappearing cause is the dependency of the normalised flux surface label $\psi_{N}(|\vec{B}|)$ on the magnitude of the magnetic field strength along a standard diagnostic line of sight (see Appendix E). Below, the features are investigated.

\section{a. Kinetic Profiles}

Each profile is strongly correlated locally over a certain domain, since the estimations of the length-scale parameters remain of the order of 0.5 (see figure $9(\mathrm{e})$ ). While this can be seen easily for $T_{e}$, the length-scale feature for $n_{e}$ is masked below $\psi_{N}=1.08$ by another high correlation feature, which is due to the incremental measurement (spectral- and, thus, spatial-wise) with the reflectometer. Above $\psi_{N}=1.08$, no correlation is found with the major part of the $n_{e}$ profile. This independence suggests that small $n_{e}$ values $\left(<5 \times 10^{16} \mathrm{~m}^{-3}\right)$ do not have an impact on the inferred edge and core profile domains. Furthermore, a third domain, captured by the $n_{e}$ profile prior, might model the outmost region better.

The length-scales $l_{n_{e} E}$ and $l_{n_{e} C}$ are correlated to some extend $(\rho=0.7)$. Furthermore, the delimiter $\psi_{N, n_{e}}$ is correlated with the major part of the density profile, especially with the edge domain $(\rho \approx 0.7)$.

The inferred $T_{e}$ and $n_{e}$ profiles seem to be inde- pendent on each other despite their interlinkage to model effective data of four diagnostics. Since $T_{e}$ is mainly determined by the first and second harmonic ranges (high optical depth) of the ECE spectra, small changes in $n_{e}$ do not affect related model predictions. In addition, the relativistic feature to predict reflectometer data seems negligible for temperatures below $1.5 \mathrm{keV}$.

\section{b. Magnetic Field and Kinetic Profiles}

The correlation between the magnetic field correction $b_{\Phi}$, representing $|\vec{B}|$, and $n_{e}$ is growing to about 0.35 towards the plasma edge, while no correlation with respect to $T_{e}$ is found. In principle, a profile and $|\vec{B}|$ can have an elevated correlation, whenever an existent large profile gradient with respect to $\psi_{N}$ is preserved after the replacement with $\psi_{N}(|\vec{B}|)$ along a diagnostic line of sight. For a tokamak like JET, $\psi_{N}(|\vec{B}|)$ is largest on the LFS, especially in the edge domain. In the particular case investigated, only the edge density has a large gradient but not the edge temperature. Hence, a change in the magnitude of the magnetic field strength demands a profile response to comply with the effective data, primarily with the one for the reflectometer in this case.

\section{c. Wall Properties and Central Temperature}

The study presented in Appendix B implies that an anti-correlation is logical for each of the parameter pairs $\left(r_{L}, p_{L}\right)$ and $\left(r_{H}, p_{H}\right)$. This is confirmed for the HFS pair ( $\rho=-0.97)$, but the LFS pair has an unexpected value of $\rho=-0.6$. Clearly, the peaking of the posterior near the meaningful limit of unity for $p_{L}$ impacts significantly the evaluation of the correlation.

The HFS wall properties correlate to some degree $\left(\rho=-0.45\right.$ for $r_{H}, \rho=0.4$ for $\left.p_{H}\right)$ with the central domain of the temperature profile. Neither MIX nor MIO has a central diagnostic line of sight, and, thus, the central profile is a bit more uncertain (see figure 9(b)). To fit the effective data, an increased central temperature can be compensated to some degree by a decrease in $r_{H}$ (less reflected power) and/or a higher $p_{H}$ (amplitude converted from $\mathrm{X}$ - to $\mathrm{O}$-mode polarisation and vice versa).

\section{d. Radiometer Sensitivity and Temperature}

Between radiometer channels, two interesting correlation features occur due to the calibration procedure. More precise, these correlations are inherited from the electron temperature profile with finite length-scale $l_{T_{e}}$ via an inhomogeneous magnetic field strength and the flux surface geometry. To understand both features, the severe anti-correlation $(-0.7 \leq \rho \leq-1)$ between certain parts of the $T_{e}$ profile and the sensitivity needs to be pointed out first. This anti-correlation follows straightforwardly for a given radiometer channel due to the di- 

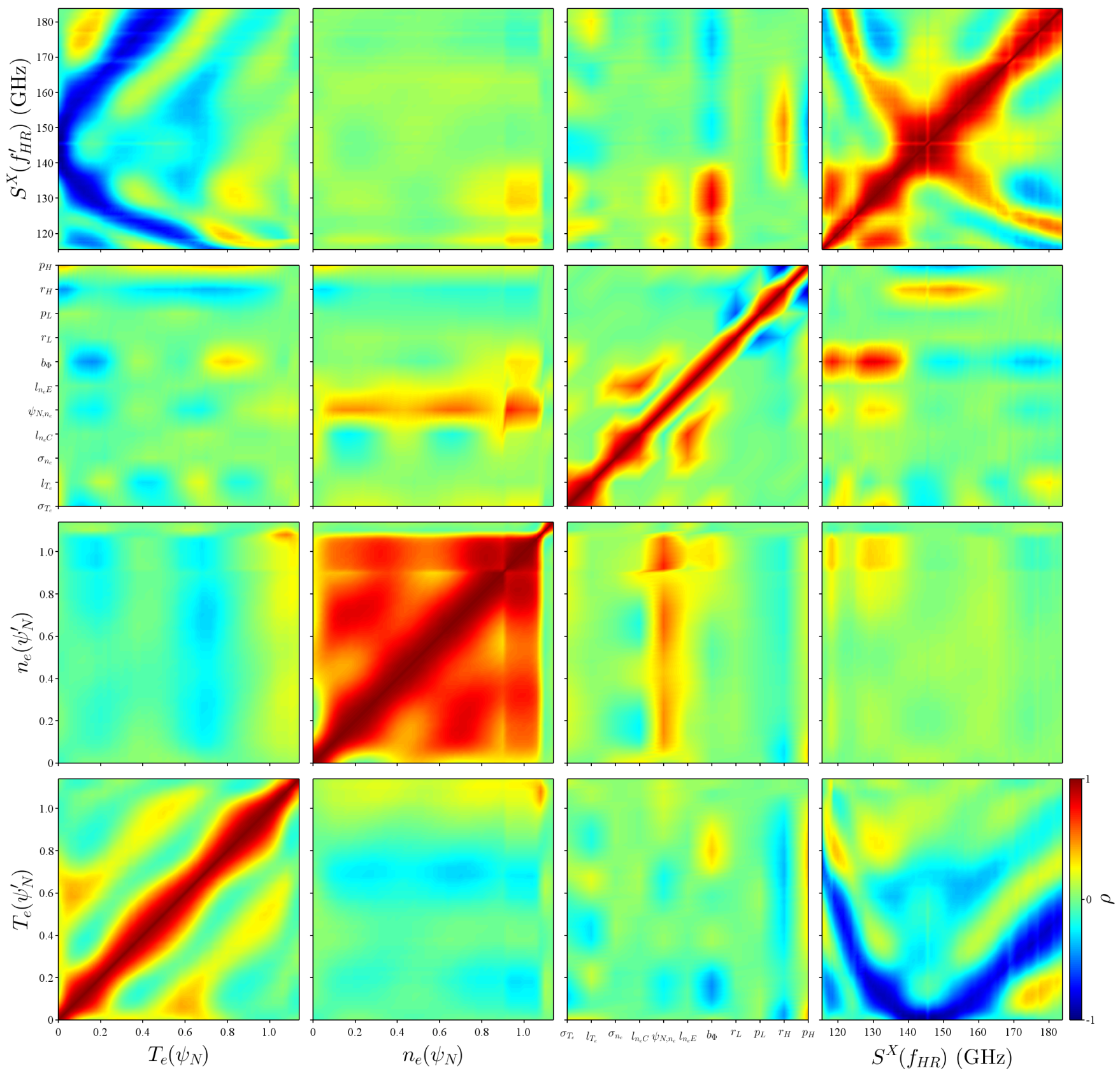

Figure 11. Posterior correlations $\rho$ between $T_{e}$ profile (58 parameters, first column and last row), $n_{e}$ profile (58 parameters, second column and third row), profile prior covariance parameters, magnetic field correction $b_{\Phi}$ and wall properties (11 parameters, third column and second row), and radiometer sensitivity $S^{X}$ (90 parameters, fourth column and first row). The correlations are obtained for the less accurate models for plasma equilibrium and ECE spectra (ECEPT).

agnostic model which includes the product of radiative temperature and sensitivity.

The first feature comprises the correlation between neighbouring radiometer channels over a spectral lengthscale of at least some $\mathrm{GHz}$, enlarging with frequency. This is the consequence of $\psi_{N}(|\vec{B}|)$, being larger for the LFS than for the HFS, which determines the transformation of $l_{T_{e}}$ to a rising length-scale with respect to $|\vec{B}|$. The trivial transfer to the probing frequency follows.
The second feature is marked by an elevated correlation of about $\rho=0.6$ between two separated spectral domains. The cause is given by the model assumption that the temperature is preserved on a given flux surface, leading to two distinct but dependent branches for the LFS and HFS, respectively. Thus, two spectral domains, like for instance close to $125 \mathrm{GHz}$ and $180 \mathrm{GHz}$, are affected in a similar way by a variation of the temperature profile. 


\section{e. Radiometer Sensitivity and Wall Properties}

The spectral domain 140 - $160 \mathrm{GHz}$, probing the central temperature profile due to the radiometer diagnosic line of sight, shows an increased (anti-)correlation above (below) $0.5(-0.5)$ with respect to $r_{H}\left(p_{H}\right)$. Both features are inherited from the connection between the HFS wall properties and central $T_{e}$ to match the MIX and MIO data described before.

\section{f. Radiometer Sensitivity, Magnetic Field and Density}

An elevated correlation is found between sensitivity in the spectral domain below $140 \mathrm{GHz}$ (LFS channels) and $|\vec{B}|$ which translates into a correlation feature regarding the edge density. A change in the magnitude of the magnetic field strength shifts the probing location of all radiometer channels in terms of flux surface label but in opposite directions for LFS and HFS channels. The corresponding temperature variation causes the channel sensitivity to adapt to fit the effective data. For this Ohmic case, $T_{e}\left(\psi_{N}\right)$ is monotonically decreasing everywhere, and, thus, LFS (HFS) channels should have a positive (negative) correlation with $|\vec{B}|$. Indeed, one finds an elevated correlation $(\rho \approx 0.7)$ for LFS channels in connection with $b_{\Phi}$ but almost no anti-correlation for the HFS channels. The change in $|\vec{B}|$ affects LFS channels much more than the HFS branch due to $\psi_{N}(|\vec{B}|)$.

Since a variation of $|\vec{B}|$ implies an adaption of the edge density explained above and $S^{X}$ of the LFS channels, the latter two quantities have an elevated correlation.

\section{Inference Using SPECE}

The investigation of the posterior is currently limited even when the parallelised version of SPECE is exploited as predictor. To reduce the computational time, the raytracing is carried out only for the central ray (single-ray setting) for each diagnostic. Hence, an antenna pattern effect is excluded. Furthermore, spectra at the diagnostic antennae of the interferometers MIX and MIO are predicted for a spectral grid with 126 equidistant nodes which is twice as fine as the one of the corresponding measured data (see bottom of figure 4). This reduces numerical uncertainties in the subsequent convolution operation to predict the probed broadband spectra.

After the parameter set is found for the MAP, conditional posteriors of individual free parameters have been surveyed. The results are compared to ECEPT findings which are used as a reference in the below brief overview. In general, good agreement is found except for the magnetic field correction and the reflection properties of the HFS wall due to the more accurate ray-tracer.

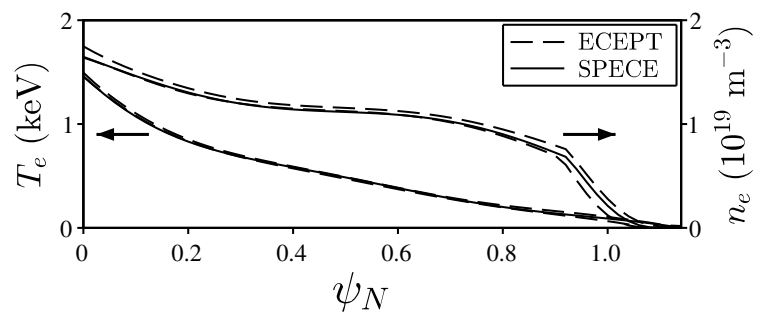

Figure 12. Electron temperature $T_{e}$ and density $n_{e}$ profiles versus normalised poloidal flux $\psi_{N}$. The profiles (solid) inferred with the ray-tracer SPECE are present at the maximum of the joint posterior. For comparison, the profiles with the two-sigma uncertainty band (dashed) are given which have been obtained after a numerical marginalisation with the less accurate predictor ECEPT for broadband ECE spectra. An acceptable agreement is found.

\section{Parameters at MAP}

The parameter set, identifying SPECE's MAP, is compared with the mean and standard deviation of ECEPT's marginal posteriors which includes its parameter set for the MAP.

\section{a. Plasma Parameters}

The profile smoothness follows from the length-scales $l_{T_{e}}=0.54, l_{n_{e} C}=0.53$ and $l_{n_{e} E}=0.17$ which are similar to ECEPT results. The profiles agree well within the two-sigma uncertainty band obtained for ECEPT towards the plasma edge (see figure 12). For the central domain though, a systematic but small decrease of $20 \mathrm{eV}$ and $2 \times 10^{17} \mathrm{~m}^{-3}$ can be found. Furthermore, $\psi_{N, n_{e}}=0.92$ is insignificantly larger but within the uncertainty of the ECEPT finding.

The correction $b_{\Phi}$ settles at $2.06 \%$ which is importantly larger by about $0.7 \%$ than the one inferred with ECEPT. The increase originates in the proper relativistic contribution to the resonance condition accounted for by the ray-tracer. In addition, the finite width of the emission layer is modelled. For the line of sights of the ECE diagnostics, this shifts each layer location radially inwards where the magnitude of the magnetic strength is higher, and a higher or lower local electron temperatures is present, depending on low-field and high-field side. Since the temperature is assumed to remain constant on a flux surface and the effective data is fixed, the more accurate model demands an additional increase in $b_{\Phi}$ with respect to the ECEPT estimation.

\section{b. Wall Reflection Properties}

The properties of the LFS have the values $r_{L}=0.34$ and $p_{L}=1.0$ comparable with ECEPT results. However, the HFS quantities $r_{H}=0.92$ and $p_{H}=0.48$ deviate heavily. This is due to the more precise description of 
(a)

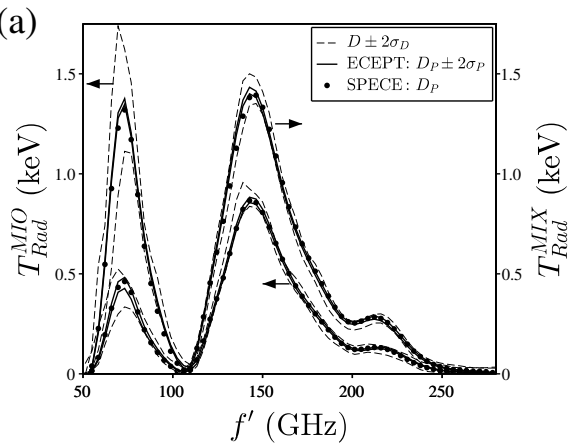

(b)

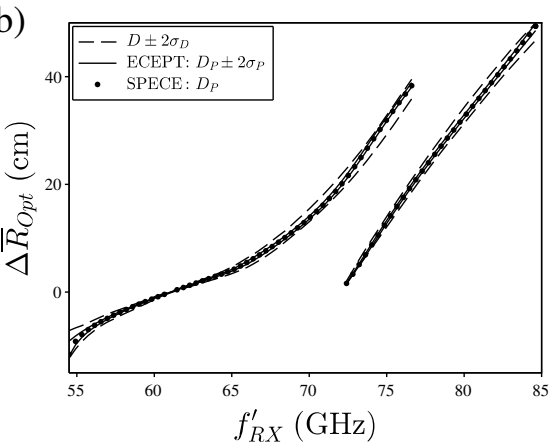

(c)

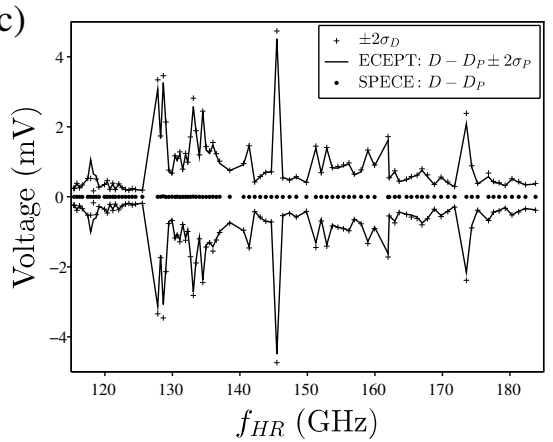

Figure 13. Comparison of predictions $D_{P}$ and effective data $D$ determined with interferometers MIX and MIO, X-mode reflectometer RX, and heterodyne radiometer HR. The two-sigma $\left(2 \sigma_{D}\right)$ confidence interval (dashed, plus symbol) of the effective data is shown. For ECEPT, the predictions, summarised by a mean plus/minus twice the standard deviation $\sigma_{P}$ (solid), follow from a large number of joint posterior samples. For SPECE, the predictions (dots) are obtained for a single parameter set for which the maximum of the joint posterior is present. (a) MIX and MIO: Radiative temperatures versus probing frequency. (b) RX: Electron density versus probing frequency. (c) HR: Difference $D-D_{P}$ versus probing frequency.

optically thin spectral domains (third harmonic and above) by SPECE. More precise, the optical thickness is captured better, and each emission layer with finite spatial extension is included. Hence, the determination of the HFS reflection properties depends critically on the accurateness of the used model to predict ECE spectra.

\section{c. Radiometer Sensitivity}

A smooth correction is obtained for the sensitivity, especially for the low-field side channels below $140 \mathrm{GHz}$. For the range 115 to $132 \mathrm{GHz}$, the correction changes from $-5 \%$ to $8 \%$. Above $145 \mathrm{GHz}$, the sensitivity is reduced by about $2 \%$. The bottom of figure 10 compares the sensitivity with the results of the standard approach and of ECEPT (implicitly). Depending on the used approach, the inferred sensitivity can vary by about $\pm 5 \%$, where the linear sensitivity model applies.

\section{Conditional Posteriors}

Any conditional posterior at the MAP has a similar width as the one found with ECEPT (some examples shown in figure 8 ). Though, the peak location might differ somewhat, being classified as systematic only for $b_{\Phi}$, $r_{H}$ and $p_{H}$ for reasons explained above.

\section{Marginal Posteriors and Correlations}

Since MCMC sampling of the posterior is not feasible time-wise, marginal posteriors and correlations cannot be investigated. Despite the systematic disagreement of the inferred HFS wall reflection properties and the magnetic field correction, SPECE results (MAP and conditional posteriors) which are numerically accessible are similar to corresponding ECEPT results. Hence, it is not expected that the missing sampling would reveal significantly different outcomes for the correlations found with ECEPT and explained by physics features. Furthermore, the missing marginal posteriors, excluding the ones for the three exceptions, are suspected to be akin to the ECEPT ones.

\section{E. Systematics due to ECEPT and SPECE}

The inference was carried for likelihoods which account only for measurement uncertainties and neglect systematic uncertainties. In the following, systematics related to the application of ECEPT or SPECE are examined. This is done by comparing model predictions with effective data sets and with model predictions for different SPECE settings like a multi-ray approach to approximate an antenna pattern. It must be mentioned that the below investigations hold only for an Ohmic JET plasma with low electron temperature and density, since the systematics depend on the plasma parameters itself due to refraction and large profile gradients, for instance.

\section{Model Using ECEPT}

Joint posterior samples for the free parameters are used to evaluate model predictions. The results and the effective data sets are shown in figure 13 where the predictions have been summarised by their mean and standard deviation. In general, predictions and data agree well inside the two-sigma uncertainty band and systematic deviations could not be identified. Thus, the implemented Bayesian model using ECEPT is able to describe effective data for an Ohmic JET plasma despite leaving aside effects like refraction and a relativistic correction in the resonance condition. However, as explained above these missing effects lead to a systematic for the inferred mag- 
netic field correction and the HFS wall reflection properties.

Another important finding is that the predictions for MIX, MIO and RX form bands of smaller width than the two-sigma uncertainty of the data. This is believed to originate in the implemented physics model and the joint inference scheme, constraining the posterior of the free parameters and their corresponding predictions. For example, the small uncertainty on $T_{R a d}^{M I X}$ and $T_{R a d}^{M I O}$ in the third harmonic range above $200 \mathrm{GHz}$ have clearly an impact on the posterior uncertainties of the inferred kinetic profiles. These uncertainties would be larger, if only first and/or second harmonic ranges of $T_{R a d}^{M I X}$ and $T_{R a d}^{M I O}$ would be the given data for the inference. Furthermore, if the reflectometer data would be given solely then the uncertainty of the inferred $n_{e}$ profile ought to comply with the uncertainty on the data.

Opposed to the findings for the other diagnostics, the spread of the predictions for HR fill the confidence interval of the effective data. This is interpreted as proof that the posterior uncertainty of the sensitivity is of the right order in magnitude.

For the overlap domain $72.4-76.6 \mathrm{GHz}$ of RX, the V (W) band predictions are slightly larger (smaller) than the mean of the effective data. Besides the demerging model aspect, a common ground remains possible within the uncertainties. Though, the diminished uncertainties of the $\mathrm{W}$ band (see bottom of figure 7) assign a higher weight to deviations from the corresponding effective data. Hence, the deviation from the mean effective data of the $\mathrm{V}$ band is larger in absolute terms.

\section{Model Using SPECE (Single-Ray)}

For the parameter set at the MAP, the predictions are well inside the uncertainty band of the effective data (see figure 13). Hence, the more accurate Bayesian model is able to capture the data. Though, minor deviations of $\pm 40 \mathrm{eV}$ exist between SPECE and ECEPT predictions mainly in the peaking of the first and second harmonic range. This systematic is much smaller than the uncertainty in the data at a given frequency. Thus, no significant systematic is introduced if SPECE is replaced by ECEPT as predictor.

A special case is at hand for the HR predictions. An almost perfect match manifests, because the sensitivity can freely adapt.

The inference was carried out for the spectral discretisation $3.66 \mathrm{GHz} / 2$, such that pre-convolution spectra are evaluated by SPECE at twice as many frequencies as the effective data sets for MIX and MIO. This discretisation was set to smaller values like $3.66 \mathrm{GHz} / 10$. A change of about $5 \mathrm{eV}$ occurs at some frequencies of the first and second harmonic range.

\section{Model Using SPECE (Multi-Ray)}

Due to the computational restrictions, SPECE had to be used in single-ray configuration. To investigate the systematic effect of a finite antenna pattern, the parameter set at the MAP was taken to predict effective data for some multi-ray approximations of a Gaussian beam. The beam waist was chosen as $2 \mathrm{~cm}$, complying with each diagnostic antenna aperture dimension, and was placed at each antenna aperture. Several multi-ray settings have been tried, and the systematic uncertainty is about $5 \mathrm{eV}$ at some frequencies in the first and second harmonic range.

\section{CONCLUSIONS AND OUTLOOK}

Two Bayesian models have been developed within the framework Minerva to infer jointly electron kinetic profiles, a magnetic field correction, wall reflection properties, and essential diagnostic sensitivity given data of four microwave diagnostics at JET. Model assumptions and aspects have been presented in detail regarding the probabilistic ansatz, plasma physics, electron cyclotron emission physics, and diagnostic principles.

One Bayesian model relies on the standard plasma equilibrium and predicts broadband ECE spectra with the ray-tracer SPECE. Due to the accurateness achieved with SPECE, the computational effort demanded to investigate the posterior for the free parameters is barely feasible even after a parallelisation of the ray-tracer was implemented. This parallelisation exploits a multitude of SPECE web services, evaluating each a spectrum for a particular spectral domain.

The less accurate predictor ECEPT was derived to evaluate broadband ECE spectra in X- and O-mode polarisations for a standard diagnostic line of sight. ECEPT neglects refraction but takes into account cut-off features, wall reflections, and emission and absorption of thermal electrons. The lowered accurateness of ECEPT reduces the computational effort tremendously, and a detailed investigation of the posterior can be carried out, relying on Markov chain Monte Carlo methods.

Both, SPECE and ECEPT, share the same assumption, that the wall reflection properties can be substantially different for low-field side wall and high-field side wall. For this generalisation, multi-pass operators have been derived analytically for low electron density under the common approximation of dealing with reflections between parallel walls. These operators are essential for the transition from single-pass to multi-pass ECE spectra and for the description of spectral domains for which the plasma is optically thin. An analytical investigation of the outcome for different harmonic ranges reveals a straightforward experimental technique to check for differing or identical wall properties, if the spectra in Xand O-mode polarisations are probed simultaneously.

Each of the four microwave diagnostic models includes 
the basic measurement principle; the spectral convolution for two Martin-Puplett interferometers, the linear mapping to the data domain of the heterodyne radiometer sensitivity and spectrum incident on the diagnostic antenna, and the demerging into spectral bands for the $\mathrm{X}$-mode reflectometer.

Both probabilistic models have more than 200 free parameters to be estimated and are tested given effective data of an Ohmic JET plasma with low electron temperature and density. This minimises systematic effects connected with the plasma equilibrium (fixed flux surface geometry) which is not inferred itself but assumed to be valid. The electron temperature and density profiles are assumed to be preserved on a given flux surface, and both profiles are modelled as independent Gaussian processes. This allows the usage of prior covariances, like the analytically derived generalised version of the squaredexponential covariance, by which length-scales can be estimated. For the example application, this involves a delimiter for the electron density profile by which the plasma core and edge domain with different length-scales can be distinguished clearly. Both profiles have been inferred extending considerably outside the last closed flux surface.

The parameter estimation gives similar results for ECEPT and SPECE. Though, the joint posterior for SPECE could not be characterised completely. At the last closed flux surface, ECEPT infers the temperature $(85 \pm 10) \mathrm{eV}$ and density $(2 \pm 0.4) \times 10^{18} \mathrm{~m}^{-3}$, and SPECE obtains $90 \mathrm{eV}$ and $2.1 \times 10^{18} \mathrm{~m}^{-3}$. However, a major difference is found for the reflection properties of the HFS wall and for the magnetic field correction. The latter is related to the relativistic resonance condition treated better by SPECE. The results for the HFS properties depend strongly on the model for the emission layer position and width approximated too crude by ECEPT. In any case, the properties of the LFS wall, diagnostic port made from Inconel, and of the HFS (ITER-like wall made from Beryllium) are significantly different. While the LFS wall reflects only a small fraction of the incoming radiation, the HFS wall reflects at least twice as much. Both walls convert considerable power fractions between perpendicular polarisation directions.

For the Ohmic plasma with the low density and temperature profiles, the correlations between parameters are accessible with ECEPT, and physics explanations have been given. For example, each of the kinetic profiles is correlated over a certain length-scale with respect to the normalised flux surface label. For the density profile, this correlation is masked by another broad feature caused by the incremental probing, spectrally and spatially, via the reflectometer. Furthermore, the vanishing correlation suggests that the outer edge density $\left(<5 \times 10^{16} \mathrm{~m}^{-3}\right)$ does not affect the outcome for the core and edge domains. No correlation is present between the density and temperature profiles despite their interlinkage to model consistently data of four diagnostics. The radiometer sensitivity is anti-correlated with the locally probed electron temperature and, hence, is correlated with neighbouring channels due to the profile length-scale and with channels which probe the plasma at the same flux surface on the opposite side of the magnetic axis.

The inference was carried out without stating any systematic uncertainties depending on plasma parameters. Some systematics have been estimated by the comparison of predictions with effective data. In some spectral domains, ECEPT and SPECE predictions deviate at a level of $40 \mathrm{eV}$ which is the largest systematic found. The finite antenna pattern and the discretisation of the pre-convoluted spectra predicted with SPECE contribute about $5 \mathrm{eV}$ each. The found systematics are usually smaller than the uncertainties on the data, and, thus, should not lead to a very different posterior if included in the inference scheme. Hence, ECEPT can assist SPECE to find faster the maximum posterior and its parameter set for an Ohmic plasma.

To investigate the joint posterior properly, the computational time for a SPECE prediction (single-ray) would need to be reduced by a factor of 20, at least. At the moment, either properly trained neural networks or a parallelisation using graphic processing units seem to be the only options towards this demand.

Other available diagnostic models, like the Lithium beam diagnostic ${ }^{20}$ at JET, could be added to the successfully tested Bayesian models. For example, edge diagnostics could reduce the derived uncertainties of the kinetic profiles. Furthermore, profile shifts between microwave diagnostics and Thomson scattering diagnostics could be investigated to determine the origin of this persistent feature at JET.

Since the electron pressure and indirectly the toroidal magnetic field was inferred jointly in this work, the flux surface geometry could be estimated by including a probabilistic equilibrium model ${ }^{17,51}$, based on the GradShafranov equation. If this could be demonstrated for an Ohmic plasma, then the joint inference could be carried out for an auxiliary-heated plasma. Not only but especially for such high density and temperature plasmas, ECEPT must be amended to capture better the finite width and position of an emission layer. This seems achievable by evaluating the local absorption coefficient directly, and the evaluation of the radiative transfer equation becomes more accurate. Multi-pass operators have been derived for the high density case but remain to be tested with ECEPT and SPECE. For the X-mode reflectometer, the relativistic $\mathrm{X}$-mode cut-off model remains valid up to at least $10 \mathrm{keV}$ but could be improved if needed.

Regarding a probabilistic equilibrium model, differing electron and ion pressures would demand additional models for the temperature and density of the relevant ion species and for associated diagnostics like the charge exchange diagnostic. Alternatively or supportingly, models for the energy stored in the plasma and for the diamagnetic loop diagnostic give information about the total 
plasma pressure. Corresponding models are currently developed.

\section{Appendix A: Generalised Squared-Exponential Covariance}

Functions, varying with the location $x$, can be captured probabilistically by a certain covariance function $\operatorname{Cov}\left(x, x^{\prime}\right)$. To describe functions which have a high correlation over a length-scale $l_{x}$, a squared-exponential covariance $^{33}$ is suitable and formally written as

$$
\begin{aligned}
& \Sigma_{S E}\left(x, x^{\prime}\right)= \\
& \sigma_{s}^{2} \exp \left(-\frac{1}{2} \frac{\left(x-x^{\prime}\right)^{2}}{l_{x}^{2}}\right)+\left\{\begin{array}{l}
\sigma^{2}: x=x^{\prime} \\
0: x \neq x^{\prime} .
\end{array}\right.
\end{aligned}
$$

While $l_{x}$ determines the smoothness, two other parameters are involved; $\sigma_{s}$ scales the process absolutely, and the positive but small $\sigma$ serves to omit numerical problems. Some sample functions, which are drawn from a multi-variate normal distribution with vanishing mean and $\Sigma_{S E}\left(x, x^{\prime}\right)$ for $l_{x}=0.1$ and $0.8\left(\sigma_{s}=1, \sigma=10^{-3}\right)$, are shown in the top and middle of figure 14(a).

To imprint several length-scales in distinct domains, the coordinate transform

$$
\begin{aligned}
& x=\mathrm{f}(y)= \\
& \left\{\begin{array}{l}
s_{1} y: x<x_{1} \\
s_{2}\left(y-y_{1}\right)+s_{1} y_{1}: x_{1} \leq x<x_{2} \\
s_{3}\left(y-y_{2}\right)+s_{2}\left(y_{2}-y_{1}\right)+s_{1} y_{1}: x_{2} \leq x<x_{3} \\
\vdots
\end{array}\right.
\end{aligned}
$$

is applied, so that at the delimiter nodes $x\left(y_{i}\right)=x_{i}$ holds. With the condition $y_{i}<y_{i+1}$, these nodes mark the limits of adjacent domains which are each compressed or stretched with respect to the corresponding $x$ domain. As stated generally in Ref. ${ }^{33}$, the generalised squaredexponential covariance is evaluated, and after some algebra

$$
\begin{aligned}
& \Sigma_{G S E}\left(y, y^{\prime}\right)=\iint \Sigma_{S E}\left(x, x^{\prime}\right) \delta(x-\mathrm{f}(y)) \delta\left(x^{\prime}-\mathrm{f}\left(y^{\prime}\right)\right) d x d x^{\prime}=\sigma_{s}^{2} \exp \left(-\frac{1}{2}\left(z(y)-z^{\prime}\left(y^{\prime}\right)\right)^{2}\right)+\left\{\begin{array}{l}
\sigma^{2}: y=y^{\prime} \\
0 \\
: y \neq y^{\prime}
\end{array}\right. \\
& \operatorname{with}\left\{\begin{array}{l}
z^{(\prime)}=y^{(\prime)} l_{y 1}^{-1}: y^{(\prime)}<y_{1} \\
z^{(\prime)}=y^{(\prime)} l_{y 2}^{-1}+y_{1}\left(l_{y 1}^{-1}-l_{y 2}^{-1}\right): y_{1} \leq y^{(\prime)}<y_{2} \\
z^{(\prime)}=y^{(\prime)} l_{y 3}^{-1}+y_{2}\left(l_{y 2}^{-1}-l_{y 3}^{-1}\right)+y_{1}\left(l_{y 1}^{-1}-l_{y 2}^{-1}\right): y_{2} \leq y^{(\prime)}<y_{3} \\
\vdots
\end{array}\right.
\end{aligned}
$$

is obtained. Thereby, each length-scale $l_{y i}=s_{i} l_{x}$ follows straightforwardly. Furthermore, if $y$ and $y^{\prime}$ locate both in the $i$ th domain, then the exponent on the far right-hand side of equation (A2) simplifies to $\left(y-y^{\prime}\right)^{2} /\left(2 l_{y i}^{2}\right)$ which resembles the one of a standard squared-exponential covariance. If locations of distinct domains are investigated, then a smooth transition between the domains is ensured by the covariance. Figure 14(b) depicts an example covariance for two length-scales $l_{x 1}=0.8(x<0.5)$ and $l_{x 2}=0.1(0.5 \leq x)$, and sample functions are shown in the bottom of figure 14(a).

To distinguish covariances of this type, $\Sigma_{G S E i}$ abbreviates a covariance for $i$ distinct domains, and the standard squared-exponential entity follows with $i=1$.

\section{Appendix B: Wall Reflections}

At first, analytical expressions are presented for the single-, double- and multi-pass quantities. With these results, a basic study for the wall properties can be un- dertaken for the low-density case. While information about the coefficients $r_{L}$ and $p_{L}$ is contained in the first harmonic range, the second harmonic range (single resonance) provides evidence for $r_{H}$ and $p_{H}$, though, dependent on $r_{L}$ and $p_{L}$. An experimental test is described, whether a simpler model, for which the LFS and HFS walls have the same properties, is more appropriate. Finally, ECE spectra, which are predicted by SPECE at the diagnostic antenna of MIX, are presented to demonstrate the influence of the wall properties.

\section{Details of Multi-Pass ECE Spectra and Operators}

Detailed derivations are listed for single-, double- and multi-pass quantities for the low-density case. Vectors and matrices used are defined in Secs. IV C 4 and IV C 5.

\section{First Harmonic Range:}


(a)

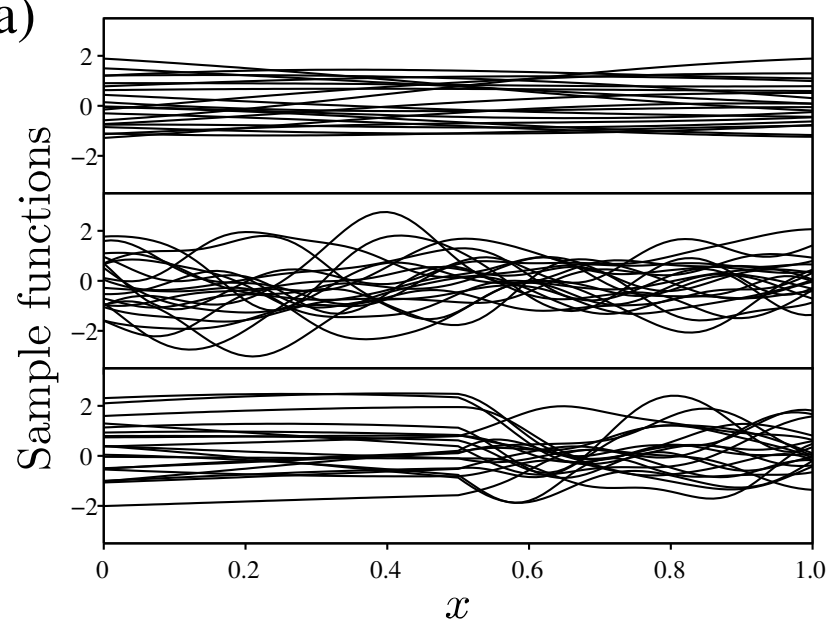

(b)

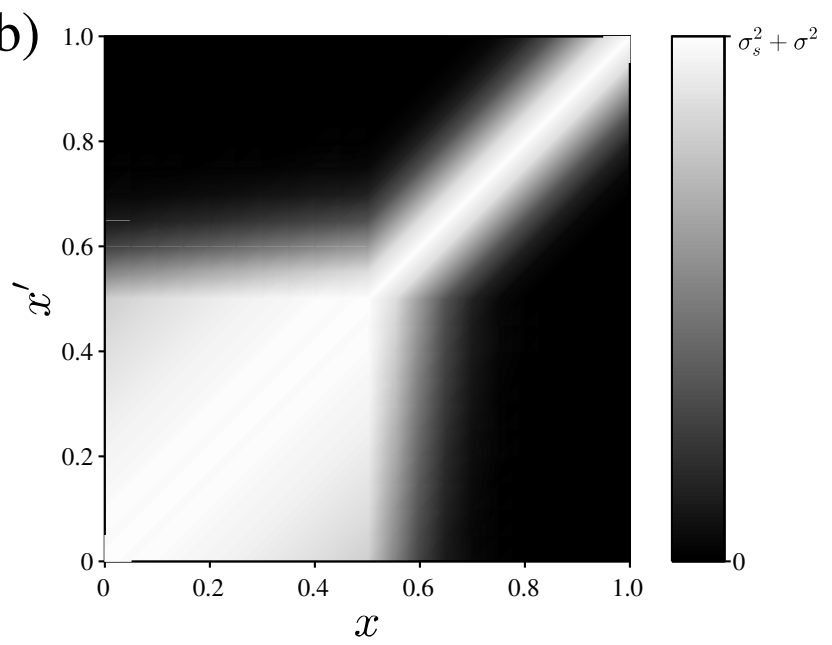

Figure 14. Aspects of squared-exponential covariance with different length-scale parameters, $\sigma_{s}=1$, and $\sigma=10^{-3}$. (a) Sample functions drawn at 101 equidistant values of $x$ from a multi-variate normal distribution with zero mean and squared-exponential covariance. Top: Single length-scale $l_{x}=0.8$. Middle: Single length-scale $l_{x}=0.1$. Bottom: Two length-scales $l_{x, 1}=0.8$ (for $x<0.5$ ) and $l_{x, 2}=0.1$ (for $0.5 \leq x$ ), determining covariance shown in (b). (b) Covariance for two length-scales $l_{x 1}$ and $l_{x 2}$ used in bottom of (a).

$$
\begin{aligned}
\vec{T}_{D} & =\mathbf{F}_{O}\left(\vec{T}_{L}^{1}+\mathcal{T}^{1} \mathbf{W}_{H} \vec{T}_{H}^{1}\right)=\left(\begin{array}{ll}
0 & 0 \\
0 & 1
\end{array}\right)\left[\left(\begin{array}{c}
T_{L}^{X 1} \\
T_{L}^{O 1}
\end{array}\right)+\left(\begin{array}{cc}
e^{-\tau^{X 1}} & 0 \\
0 & e^{-\tau^{O 1}}
\end{array}\right) r_{H}\left(\begin{array}{cc}
1-p_{H} & p_{H} \\
p_{H} & 1-p_{H}
\end{array}\right)\left(\begin{array}{c}
T_{H}^{X 1} \\
T_{H}^{O 1}
\end{array}\right)\right] \\
& =\left(\begin{array}{c}
0 \\
T_{L}^{O 1}
\end{array}\right)+e^{-\tau^{O 1}} r_{H}\left(\begin{array}{cc}
0 & 0 \\
p_{H} & 1-p_{H}
\end{array}\right)\left(\begin{array}{c}
T_{H}^{X 1} \\
T_{H}^{O 1}
\end{array}\right)=\left(\begin{array}{c}
0 \\
T_{L}^{O 1}+e^{-\tau^{O 1}} r_{H}\left[p_{H} T_{H}^{X 1}+\left(1-p_{H}\right) T_{H}^{O 1}\right]
\end{array}\right)
\end{aligned}
$$

$$
\begin{aligned}
& \mathbf{F}_{O} \mathcal{T}^{1} \mathbf{W}_{H} \mathcal{T}^{1} \mathbf{F}_{O} \mathbf{W}_{L}= \\
& =\left(\begin{array}{ll}
0 & 0 \\
0 & 1
\end{array}\right)\left(\begin{array}{cc}
e^{-\tau^{X 1}} & 0 \\
0 & e^{-\tau^{O 1}}
\end{array}\right) r_{H}\left(\begin{array}{cc}
1-p_{H} & p_{H} \\
p_{H} & 1-p_{H}
\end{array}\right)\left(\begin{array}{cc}
e^{-\tau^{X 1}} & 0 \\
0 & e^{-\tau^{O 1}}
\end{array}\right)\left(\begin{array}{ll}
0 & 0 \\
0 & 1
\end{array}\right) r_{L}\left(\begin{array}{cc}
1-p_{L} & p_{L} \\
p_{L} & 1-p_{L}
\end{array}\right) \\
& =r_{H} r_{L}\left(\begin{array}{cc}
0 & 0 \\
e^{-\tau^{O 1}} p_{H} & e^{-\tau^{O 1}}\left(1-p_{H}\right)
\end{array}\right)\left(\begin{array}{cc}
0 & 0 \\
e^{-\tau^{O 1}} p_{L} & e^{-\tau^{O 1}}\left(1-p_{L}\right)
\end{array}\right)=r_{H} r_{L} e^{-2 \tau^{O 1}}\left(1-p_{H}\right)\left(\begin{array}{cc}
0 & 0 \\
p_{L} & 1-p_{L}
\end{array}\right) \\
& \mathbf{F}_{X} \mathbf{W}_{L}=\left(\begin{array}{ll}
1 & 0 \\
0 & 0
\end{array}\right) r_{L}\left(\begin{array}{cc}
1-p_{L} & p_{L} \\
p_{L} & 1-p_{L}
\end{array}\right)=r_{L}\left(\begin{array}{cc}
1-p_{L} & p_{L} \\
0 & 0
\end{array}\right) \\
& \mathbf{M}_{D}=\mathbf{F}_{O} \mathcal{T}^{1} \mathbf{W}_{H} \mathcal{T}^{1} \mathbf{F}_{O} \mathbf{W}_{L}+\mathbf{F}_{X} \mathbf{W}_{L}=r_{L}\left(\begin{array}{cc}
1-p_{L} & p_{L} \\
r_{H} e^{-2 \tau^{O 1}}\left(1-p_{H}\right) p_{L} & r_{H} e^{-2 \tau^{O 1}}\left(1-p_{H}\right)\left(1-p_{L}\right)
\end{array}\right) \\
& \mathbf{M}=\frac{1}{D_{1}}\left(\begin{array}{cc}
1-r_{L} r_{H} e^{-2 \tau^{O 1}}\left(1-p_{H}\right)\left(1-p_{L}\right) & r_{L} p_{L} \\
r_{L} r_{H} e^{-2 \tau^{O 1}}\left(1-p_{H}\right) p_{L} & 1-r_{L}\left(1-p_{L}\right)
\end{array}\right) \\
& D_{1}=\left[1-r_{L}\left(1-p_{L}\right)\right]\left[1-r_{L} r_{H} e^{-2 \tau^{O 1}}\left(1-p_{H}\right)\left(1-p_{L}\right)\right]-r_{L}^{2} p_{L}^{2} r_{H} e^{-2 \tau^{O 1}}\left(1-p_{H}\right)
\end{aligned}
$$

For the special case with $r_{L}=1$ and $p_{L}=0$, both above equations diverge. Removing the divergent contribution gives

$$
\mathbf{M}=\left(\begin{array}{cc}
1 & 0 \\
0 & \frac{1}{1-r_{H} e^{-2 \tau^{O 1}}\left(1-p_{H}\right)}
\end{array}\right)
$$


For SPECE, the above quantities are amended with the finite emission and absorption for the X-mode component.

Second Harmonic Ranges - Single Resonance:

$$
\begin{aligned}
& \vec{T}_{D}=\vec{T}_{L}^{2}+\mathcal{T}^{2} \mathbf{W}_{H} \vec{T}_{H}^{2}=\left(\begin{array}{c}
T_{L}^{X 2} \\
T_{L}^{O 2}
\end{array}\right)+\left(\begin{array}{cc}
e^{-\tau^{X 2}} & 0 \\
0 & e^{-\tau^{O 2}}
\end{array}\right) r_{H}\left(\begin{array}{cc}
1-p_{H} & p_{H} \\
p_{H} & 1-p_{H}
\end{array}\right)\left(\begin{array}{c}
T_{H}^{X 2} \\
T_{H}^{O 2}
\end{array}\right) \\
& =\left(\begin{array}{c}
T_{L}^{X 2}+e^{-\tau^{X 2}} r_{H}\left[\left(1-p_{H}\right) T_{H}^{X 2}+p_{H} T_{H}^{O 2}\right] \\
T_{L}^{O 2}+e^{-\tau^{O 2}} r_{H}\left[\left(1-p_{H}\right) T_{H}^{O 2}+p_{H} T_{H}^{X 2}\right]
\end{array}\right) \\
& \mathbf{M}_{D}=\mathcal{T}^{2} \mathbf{W}_{H} \mathcal{T}^{2} \mathbf{W}_{L}= \\
& =\left(\begin{array}{cc}
e^{-\tau^{X 2}} & 0 \\
0 & e^{-\tau^{O 2}}
\end{array}\right) r_{H}\left(\begin{array}{cc}
1-p_{H} & p_{H} \\
p_{H} & 1-p_{H}
\end{array}\right)\left(\begin{array}{cc}
e^{-\tau^{X 2}} & 0 \\
0 & e^{-\tau^{O 2}}
\end{array}\right) r_{L}\left(\begin{array}{cc}
1-p_{L} & p_{L} \\
p_{L} & 1-p_{L}
\end{array}\right) \\
& =r_{H} r_{L}\left(\begin{array}{cc}
e^{-2 \tau^{X 2}}\left(1-p_{H}\right)\left(1-p_{L}\right)+e^{-\left(\tau^{X 2}+\tau^{O 2}\right)} p_{H} p_{L} & e^{-2 \tau^{X 2}}\left(1-p_{H}\right) p_{L}+e^{-\left(\tau^{X 2}+\tau^{O 2}\right)} p_{H}\left(1-p_{L}\right) \\
e^{-\left(\tau^{X 2}+\tau^{O 2}\right)} p_{H}\left(1-p_{L}\right)+e^{-2 \tau^{O 2}}\left(1-p_{H}\right) p_{L} & e^{-\left(\tau^{X 2}+\tau^{O 2}\right)} p_{H} p_{L}+e^{-2 \tau^{O 2}}\left(1-p_{H}\right)\left(1-p_{L}\right)
\end{array}\right) \\
& D_{2}=\left(1-r_{H} r_{L}\left[e^{-2 \tau^{X 2}}\left(1-p_{H}\right)\left(1-p_{L}\right)+e^{-\left(\tau^{X 2}+\tau^{O 2}\right)} p_{H} p_{L}\right]\right)\left(1-r_{H} r_{L}\left[e^{-\left(\tau^{X 2}+\tau^{O 2}\right)} p_{H} p_{L}+e^{-2 \tau^{O 2}}\left(1-p_{H}\right)\left(1-p_{L}\right)\right]\right) \\
& -r_{H}^{2} r_{L}^{2}\left[e^{-2 \tau^{X 2}}\left(1-p_{H}\right) p_{L}+e^{-\left(\tau^{X 2}+\tau^{O 2}\right)} p_{H}\left(1-p_{L}\right)\right]\left[e^{-\left(\tau^{X 2}+\tau^{O 2}\right)} p_{H}\left(1-p_{L}\right)+e^{-2 \tau^{O 2}}\left(1-p_{H}\right) p_{L}\right]
\end{aligned}
$$

\section{Overlapping Second and Third Harmonic Ranges:}

Other overlapping domains for higher spectral ranges are omitted here but can be derived accordingly.

$$
\begin{aligned}
& \vec{T}_{L}=\vec{T}_{L}^{3}+\mathcal{T}^{3} \vec{T}_{L}^{2}=\left(\begin{array}{c}
T_{L}^{X 3} \\
T_{L}^{O 3}
\end{array}\right)+\left(\begin{array}{cc}
e^{-\tau^{X 3}} & 0 \\
0 & e^{-\tau^{O 3}}
\end{array}\right)\left(\begin{array}{c}
T_{L}^{X 2} \\
T_{L}^{O 2}
\end{array}\right)=\left(\begin{array}{c}
T_{L}^{X 3}+e^{-\tau^{X 3}} T_{L}^{X 2} \\
T_{L}^{O 3}+e^{-\tau^{O 3}} T_{L}^{O 2}
\end{array}\right) \\
& \vec{T}_{H}=\vec{T}_{H}^{2}+\mathcal{T}^{2} \vec{T}_{L}^{3}=\left(\begin{array}{c}
T_{H}^{X 2} \\
T_{H}^{O 2}
\end{array}\right)+\left(\begin{array}{cc}
e^{-\tau^{X 2}} & 0 \\
0 & e^{-\tau^{O 2}}
\end{array}\right)\left(\begin{array}{c}
T_{H}^{X 3} \\
T_{H}^{O 3}
\end{array}\right)=\left(\begin{array}{c}
T_{H}^{X 2}+e^{-\tau^{X 2}} T_{H}^{X 3} \\
T_{H}^{O 2}+e^{-\tau^{O 2}} T_{H}^{O 3}
\end{array}\right) \\
& \vec{T}_{D}=\vec{T}_{L}+\mathcal{T}^{3} \mathcal{T}^{2} \mathbf{W}_{H} \vec{T}_{H}=\vec{T}_{L}^{3}+\mathcal{T}^{3} \vec{T}_{L}^{2}+\mathcal{T}^{3} \mathcal{T}^{2} \mathbf{W}_{H}\left(\vec{T}_{H}^{2}+\mathcal{T}^{2} \vec{T}_{L}^{3}\right)= \\
& =\left(\begin{array}{c}
T_{L}^{X 3}+e^{-\tau^{X 3}} T_{L}^{X 2} \\
T_{L}^{O 3}+e^{-\tau^{O 3}} T_{L}^{O 2}
\end{array}\right)+\left(\begin{array}{cc}
e^{-\tau^{X 2}} & 0 \\
0 & e^{-\tau^{O 2}}
\end{array}\right) r_{H}\left(\begin{array}{cc}
1-p_{H} & p_{H} \\
p_{H} & 1-p_{H}
\end{array}\right)\left(\begin{array}{c}
T_{H}^{X 2}+e^{-\tau^{X 2}} T_{H}^{X 3} \\
T_{H}^{O 2}+e^{-\tau^{O 2}} T_{H}^{O 3}
\end{array}\right) \\
& =\left(\begin{array}{l}
T_{L}^{X 3}+e^{-\tau^{X 3}} T_{L}^{X 2}+r_{H} e^{-\tau^{X 2}}\left[\left(1-p_{H}\right)\left(T_{H}^{X 2}+e^{-\tau^{X 2}} T_{H}^{X 3}\right)+p_{H}\left(T_{H}^{O 2}+e^{-\tau^{O 2}} T_{H}^{O 3}\right)\right] \\
T_{L}^{O 3}+e^{-\tau^{O 3}} T_{L}^{O 2}+r_{H} e^{-\tau^{O 2}}\left[\left(1-p_{H}\right)\left(T_{H}^{O 2}+e^{-\tau^{O 2}} T_{H}^{O 3}\right)+p_{H}\left(T_{H}^{X 2}+e^{-\tau^{X 2}} T_{H}^{X 3}\right)\right]
\end{array}\right)
\end{aligned}
$$

$$
\begin{aligned}
& \mathbf{M}_{D}=\mathcal{T}^{3} \mathcal{T}^{2} \mathbf{W}_{H} \mathcal{T}^{2} \mathcal{T}^{3} \mathbf{W}_{L} \\
& =\left[\prod_{i=2}^{i=3}\left(\begin{array}{cc}
e^{-\tau^{X i}} & 0 \\
0 & e^{-\tau^{O i}}
\end{array}\right)\right] r_{H}\left(\begin{array}{cc}
1-p_{H} & p_{H} \\
p_{H} & 1-p_{H}
\end{array}\right)\left[\prod_{i=2}^{i=3}\left(\begin{array}{cc}
e^{-\tau^{X i}} & 0 \\
0 & e^{-\tau^{O i}}
\end{array}\right)\right] r_{L}\left(\begin{array}{cc}
1-p_{L} & p_{L} \\
p_{L} & 1-p_{L}
\end{array}\right) \\
& =r_{H} r_{L}\left(\begin{array}{c}
e^{-2 \sum_{i=2}^{i=3} \tau^{X i}}\left(1-p_{H}\right)\left(1-p_{L}\right)+e^{-\sum_{i=2}^{i=3}\left(\tau^{X i}+\tau^{O i}\right)} p_{H} p_{L} e^{-2 \sum_{i=2}^{i=3} \tau^{X i}}\left(1-p_{H}\right) p_{L}+e^{-\sum_{i=2}^{i=3}\left(\tau^{X i}+\tau^{O i}\right)} p_{H}\left(1-p_{L}\right) \\
e^{-\sum_{i=2}^{i=3}\left(\tau^{X i}+\tau^{O i}\right)} p_{H}\left(1-p_{L}\right)+e^{-2 \sum_{i=2}^{i=3} \tau^{O i}}\left(1-p_{H}\right) p_{L} e^{-\sum_{i=2}^{i=3}\left(\tau^{X i}+\tau^{O i}\right)} p_{H} p_{L}+e^{-2 \sum_{i=2}^{i=3} \tau^{O i}}\left(1-p_{H}\right)\left(1-p_{L}\right)
\end{array}\right)
\end{aligned}
$$




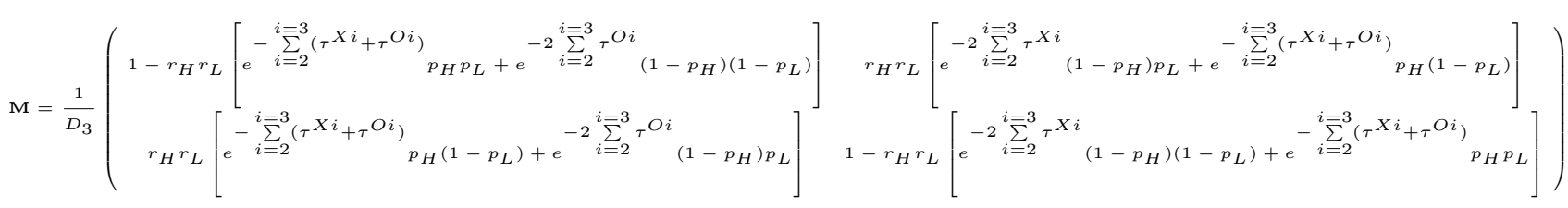

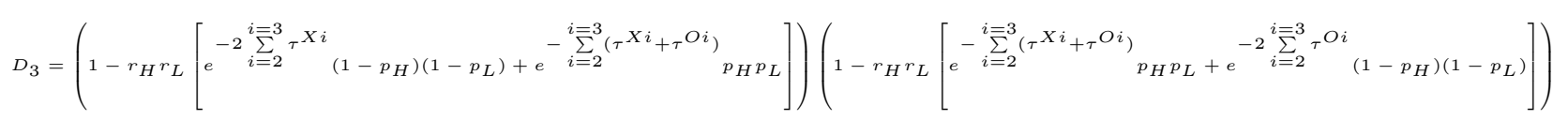

$$
\begin{aligned}
& -r_{H}^{2} r_{L}^{2}\left[e^{-2 \sum_{i=2}^{i=3} \tau^{X i}}\left(1-p_{H}\right) p_{L}+e^{-\sum_{i=2}^{i=3}\left(\tau^{X i}+\tau^{O i}\right)} p_{H}\left(1-p_{L}\right)\right]\left[e^{-\sum_{i=2}^{i=3}\left(\tau^{X i}+\tau^{O i}\right)} p_{H}\left(1-p_{L}\right)+e^{-2 \sum_{i=2}^{i=3} \tau^{O i}}\left(1-p_{H}\right) p_{L}\right]
\end{aligned}
$$

\section{Condition on Properties of Low-Field Side Wall}

The components of $\vec{T}_{R a d}^{A}$ for the first harmonic range (see equation (21)) reveal information about $r_{L}$ and $p_{L}$, as the ratio

$$
R_{1}=\frac{T_{R a d A}^{X 1}}{T_{\text {RadA }}^{O 1}}=\frac{r_{L} p_{L}}{1-r_{L}\left(1-p_{L}\right)}
$$

demonstrates. This operation removes any dependency on plasma parameters. A simple inversion finds the condition

$$
p_{L}=\frac{R_{1}}{1-R_{1}} \frac{1-r_{L}}{r_{L}}
$$

in the parameter plane.

The above condition implies that a single solution for $r_{L}$ and $p_{L}$ does not exist but a curve with a $p_{L} \propto 1 / r_{L}$ dependence. Furthermore, a lower limit $r_{L}=T_{\text {RadA }}^{X 1} / T_{\text {RadA }}^{O 1}$ is at hand, when the meaningful maximum $p_{L}=1$ is set. Demanding $r_{L}=1, p_{L}$ approaches 0 . For a JET-like example with $R_{1}=1 / 3$ (see bottom of figure 4 ), the condition is shown in figure 15 .

\section{Condition on Properties of High-Field Side Wall}

For the optically thin case $\left(\tau^{O 2} \ll 1, T_{L}^{O 2}=T_{H}^{O 2}=0\right)$ for O-mode waves and for a large enough optical depth for $\mathrm{X}$-mode waves $\left(\tau^{X 2} \gg 1\right)$, the matrix stated by equation (B10) becomes

$\mathbf{M}=$

$\frac{1}{1-r_{H} r_{L}\left(1-p_{H}\right)\left(1-p_{L}\right)}\left(\begin{array}{cc}1-r_{H} r_{L}\left(1-p_{H}\right)\left(1-p_{L}\right) & 0 \\ r_{H} r_{L}\left(1-p_{H}\right) p_{L} & 1\end{array}\right)$

Furthermore, the double-pass spectrum given by equation (B8) reduces to

$$
\vec{T}_{D}=\left(\begin{array}{c}
T_{L}^{X 2} \\
r_{H} p_{H} T_{H}^{X 2}
\end{array}\right)
$$

and the radiative temperatures $T_{L}^{X 2}=T_{H}^{X 2}$ equal for a Maxwellian plasma at low-temperature. Altogether, the multi-pass spectrum is evaluated as

$$
\vec{T}_{R a d A}=\mathbf{M} \vec{T}_{D}=T_{L}^{X 2}\left(\begin{array}{c}
1 \\
\frac{r_{H} r_{L}\left(1-p_{H}\right) p_{L}+r_{H} p_{H}}{1-r_{H} r_{L}\left(1-p_{H}\right)\left(1-p_{L}\right)}
\end{array}\right) .
$$

The ratio $R_{2}=T_{R a d A}^{O 2} / T_{R a d A}^{X 2}$ of the components gives

$$
R_{2}=\frac{r_{H} r_{L}\left(1-p_{H}\right) p_{L}+r_{H} p_{H}}{1-r_{H} r_{L}\left(1-p_{H}\right)\left(1-p_{L}\right)} .
$$

Inverting for $p_{H}$ yields

$$
p_{H}=\frac{u}{1-u} \frac{R_{2} / u-r_{H}}{r_{H}}
$$

with $u=r_{L}\left(p_{L}+R_{2}\left(1-p_{L}\right)\right)$, and after inserting condition (B19)

$$
p_{H}=\frac{R_{2}\left(1-R_{1}\right)-r_{H}\left[r_{L}\left(R_{2}-R_{1}\right)+R_{1}\left(1-R_{2}\right)\right]}{r_{H}\left(1-R_{1}\right)-r_{H}\left[r_{L}\left(R_{2}-R_{1}\right)+R_{1}\left(1-R_{2}\right)\right]}
$$

remains. Opposed to the condition for the low-field side quantities, the above condition fills a whole domain in the $r_{H-} p_{H}$ plane, when $r_{L}$ is varied and $R_{1}$ and $R_{2}$ are kept constant. This is shown in figure 15 for a JET-like example with $R_{1}=1 / 3$ and $R_{2}=2 / 3$.

From condition (B24), it is easy to see that a lower limit $r_{H}=R_{2}$ exists, when $p_{H}=1$ is set. Furthermore, this lower limit is independent on the allowed combinations of $r_{L}$ and $p_{L}$ presented in figure 15. On the contrary, as $r_{H}$ approaches unity, $p_{H}$ can take values between 0 and 0.5 for the example dependent on valid $r_{L}$ and $p_{L}$ pairs.

It must be mentioned that the possible combination $r_{H}=r_{L}=1$ and $p_{H}=p_{L}=0$ marks a perfectly reflecting inner wall, decoupling the polarisation directions. Furthermore, this parameter combination is the only solution for which HFS and LFS walls have the same properties. 


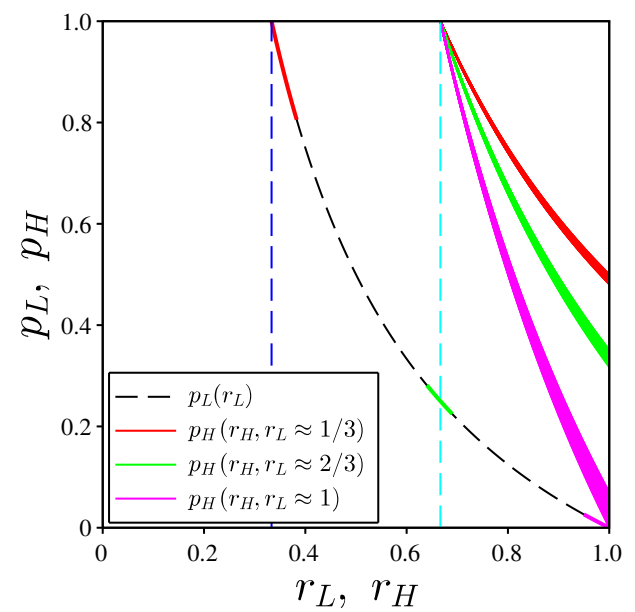

Figure 15. Study of reflection coefficients $\left(r_{L}\right.$ and $\left.r_{H}\right)$ and polarisation-scrambling coefficients $\left(p_{L}\right.$ and $\left.p_{H}\right)$ associated with low- and high-field sides. From the ratios $R_{1(2)}=$ $T_{\text {RadA }}^{X 1(O 2)} / T_{\text {RadA }}^{O 1(X 2)}$ of the radiative temperatures of the ECE spectra in X- and O-mode polarisation at the LFS diagnostic antenna, two conditions (see equations (B19) and (B24)) are derived. For the JET-like example $R_{1}=1 / 3$ and $R_{2}=2 / 3$ (see bottom of figure 4), $p_{L} \propto 1 / r_{L}$ (black-dashed) is obtained, and a lower limit $r_{L} \geq R_{1}$ (blue-dashed) follows. The second condition $p_{H} \propto 1 / r_{H}$ fills a whole domain, as $r_{H}$ approaches unity and $r_{L}$ and $p_{L}$ are varied (red, green and magenta). A common lower limit is demanded $r_{H} \geq R_{2}$ (cyandashed) for $p_{H}=1$.

\section{Pinning Down Reflections Properties}

The two conditions (B19) and (B24) derived do not pin down the wall properties in the parameter space. A lengthy but careful analytical investigation of equations (B14) and (B16), which is skipped here, reveals that the spectra of the third (and higher) harmonic range are mandatory to fix the reflections properties. Since the plasma is optically thin for these spectral ranges, the accurate optical thickness and emission layer shape are essential. Both are connected to the local electron density, and, thus, reliable density information needs to be at hand.

\section{Experimental Test of Wall Property Models}

A common assumption in the field of ECE diagnostics states that the HFS and LFS walls have the same properties, and, thus, the special case $r_{L}=r_{H}$ and $p_{L}=p_{H}$ is imposed. An experimental test is possible whether this simplified model might be appropriate. For this test, the wave mode amplitudes in X- and O-mode polarisations need to be probed in different harmonic ranges for a low density plasma.
Equation (B22) simplifies to

$$
\vec{T}_{\text {RadA }}=T_{L}^{X 2}\left(\begin{array}{c}
1 \\
\frac{r_{L} p_{L}}{1-r_{L}\left(1-p_{L}\right)}
\end{array}\right) .
$$

The ratio $R_{2}=r_{L} p_{L} /\left[1-r_{L}\left(1-p_{L}\right)\right]$ follows which implies $R_{1}=R_{2}$, according to equation (B18). As a consequence, the wave mode amplitudes in the first and second harmonic ranges have the same behaviour in relative terms. Hence, the comparison for the ratios $R_{1}$ and $R_{2}$ carry information about distinct or similar wall reflection properties. At JET, $R_{1} \neq R_{2}$ is evident shown in bottom of figure 4 .

If $R_{1}=R_{2}$ holds, then condition (B24) becomes independent of the LFS properties, and its form is similar to condition (B19). Hence, the LFS and HFS properties could be still substantially different, and the third harmonic range needs to be investigated to obtain a final answer. The above is presented graphically in figure 16 .

\section{Scanning Wall Properties: SPECE Predictions}

For different sets of wall properties, SPECE predictions are made to show the variation of ECE spectra in $\mathrm{X}$ - and $\mathrm{O}$-mode polarisations incident on the diagnostic antenna of MIX. All other input parameters are taken as inferred in Sec. VID for the low-density case and are kept constant.

Three special cases are shown in figure 16(a) for totally absorbing walls, for totally reflecting walls with no and complete polarisation scrambling. If the wall absorbs all incident spectra, then only the direct plasma emission is incident on the antenna, and the O-mode component vanishes almost in the second and third harmonic ranges for the low-density case. These ranges for which the plasma is very optically thin are enhanced tremendously by totally reflecting walls when the polarisation directions are decoupled. Furthermore, the X-mode component in the first harmonic range disappears. This component has the same amplitude as the O-mode component when the LFS wall scrambles fully the amplitudes.

Figure 16(b) presents a JET-like case for which the ratios $R_{1}=1 / 3$ and $R_{2}=2 / 3$ determine the used conditions (B19) and (B24) to vary jointly the wall properties. While the components in the first and second harmonic ranges change little, the amplitudes of the third harmonic range are influenced most especially towards a decoupling totally reflecting LFS wall.

If LFS and HFS properties remain independent but follow similar conditions with $R_{1}=R_{2}=1 / 3$, the predictions for first harmonic and second harmonic ranges have similar amplitudes when $\mathrm{X}$ - and O-mode components are exchanged (see figure 16(c)). Also for this case, the spread in the third harmonic range is considerable.

Assuming identical LFS and HFS properties for $R_{1}=$ $R_{2}=1 / 3$, the spectra are shown in figure 16(d) for three parameter combinations. Similar broadband spectra can 
(a)

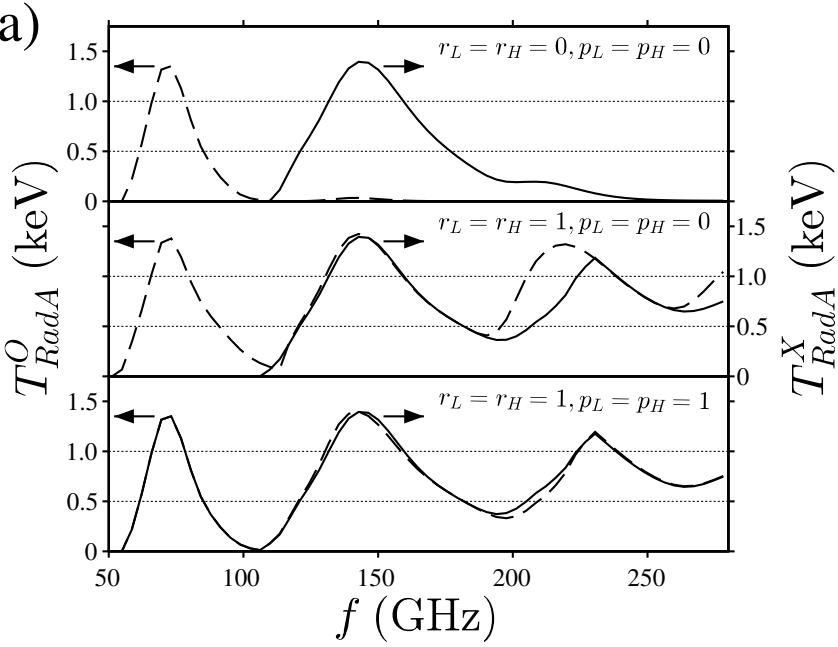

(b)

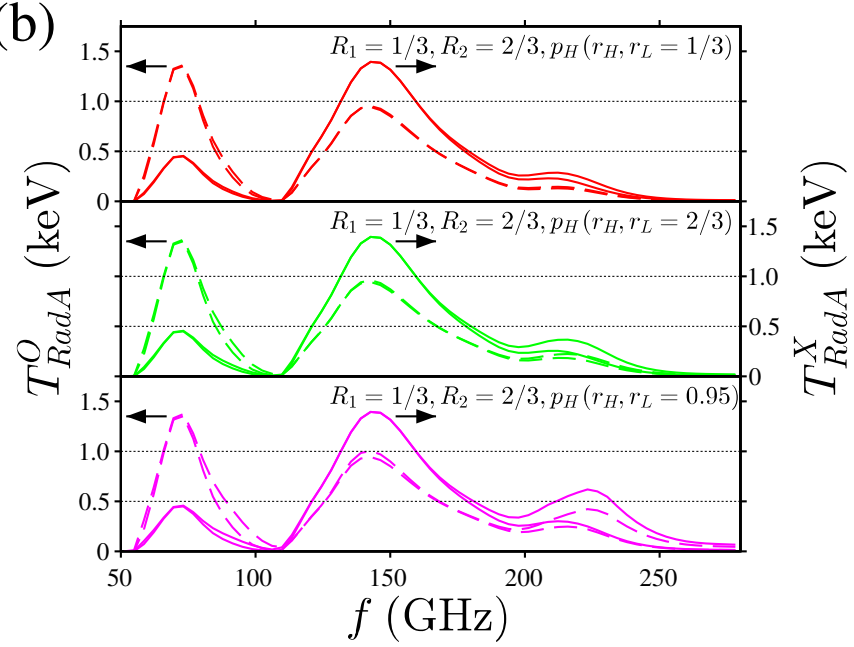

(c)

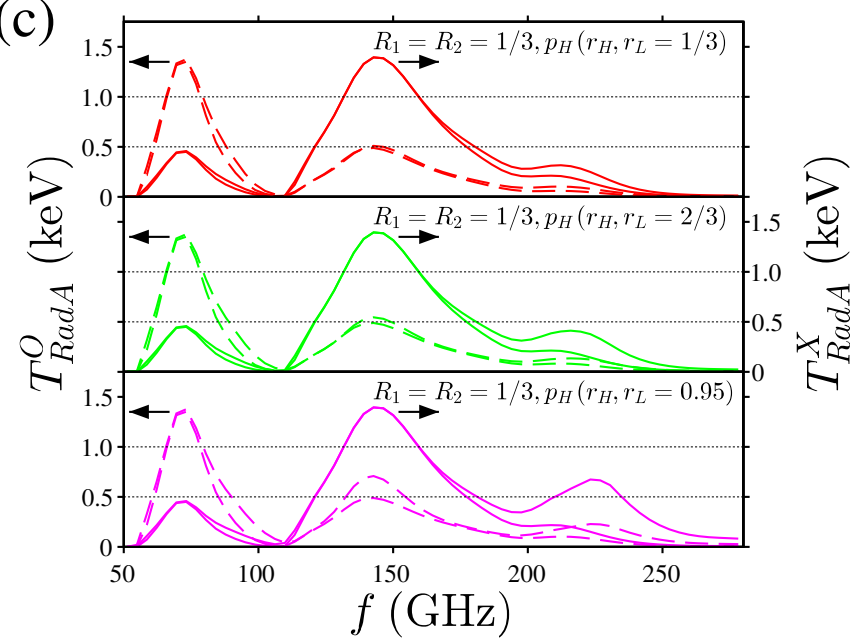

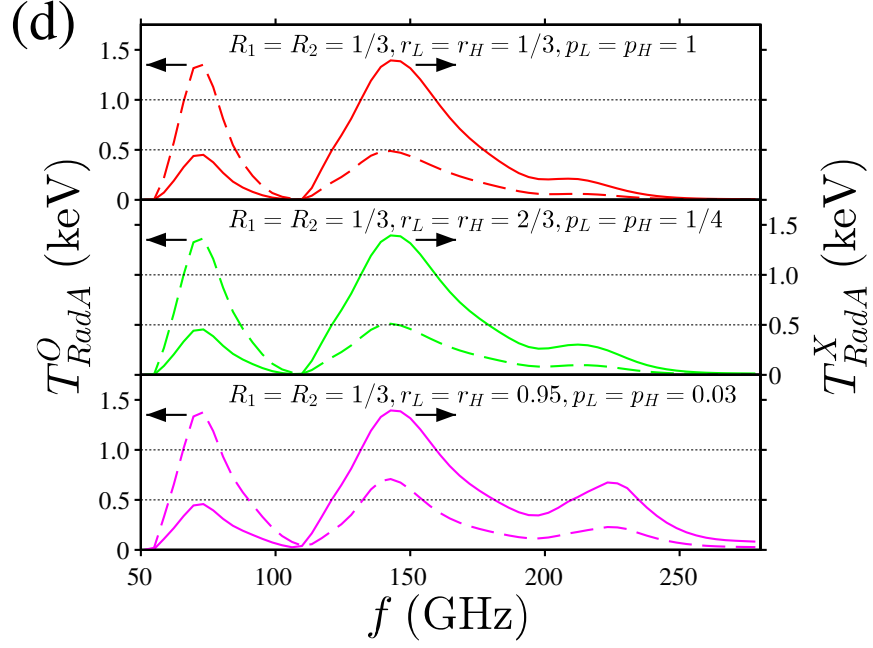

Figure 16. Broadband ECE spectra in X-mode $\left(T_{\text {RadA }}^{X}\right.$, solid) and O-mode polarisation $\left(T_{\text {RadA }}^{O}\right.$, dashed $)$ predicted by raytracer SPECE for different combinations of wall reflection properties $r_{L}, p_{L}$ (low-field side, LFS), $r_{H}$ and $p_{H}$ (high-field side, HFS). The spectra are predicted at the antenna aperture of the MIX diagnostic for a low density plasma in JET. While (a) shows special cases, (b) - (d) (see colour coding of figure 15) present predictions for the conditions (B19) and (B24) on the properties, such that the ratio $R_{1}=T_{R a d A}^{X} / T_{\text {RadA }}^{O}=1 / 3$ in the first harmonic range is typical for a low density plasma in JET. (a) Top: Totally absorbing walls. Middle: Totally reflecting walls decoupling polarisation directions. Bottom: Totally reflecting walls coupling polarisation directions entirely. (b) Distinct LFS and HFS properties chosen by conditions for ratio $R_{2}=T_{\text {RadA }}^{O} / T_{\text {RadA }}^{X}=2 / 3$ (evident at JET shown in bottom of figure 4 ) in second harmonic range. All possible property combinations cause a spread, especially in the third harmonic range. (c) Distinct LFS and HFS properties chosen by conditions for ratio $R_{1}=R_{2}=1 / 3$. Corresponding property combinations impact heavily the spectra in third harmonic range. (d) Same LFS and HFS properties chosen by conditions for ratio $R_{1}=R_{2}=1 / 3$. A wide range of property combinations results in similar spectra.

be obtained along the condition (B19). A decoupling and totally reflecting wall, however, increases the amplitudes significantly in the third harmonic range.

\section{Appendix C: Parallelisation of SPECE}

A probabilistic analysis quantifies the plausibility of combinations of free parameters given a model and measured data with uncertainties. This kind of inference has two aspects which determine its duration; the total number of free parameters and data points (dimensionality), and the computational time for a single model prediction. The dimensionality is computationally manageable by a single PC even for several hundred parameters and data points at least, if the second one is negligible. However, a complex model might imply a long computational time, delaying an inference procedure.

When the dimensionality is fixed by a scientific problem, the model complexity could be reduced, and/or 


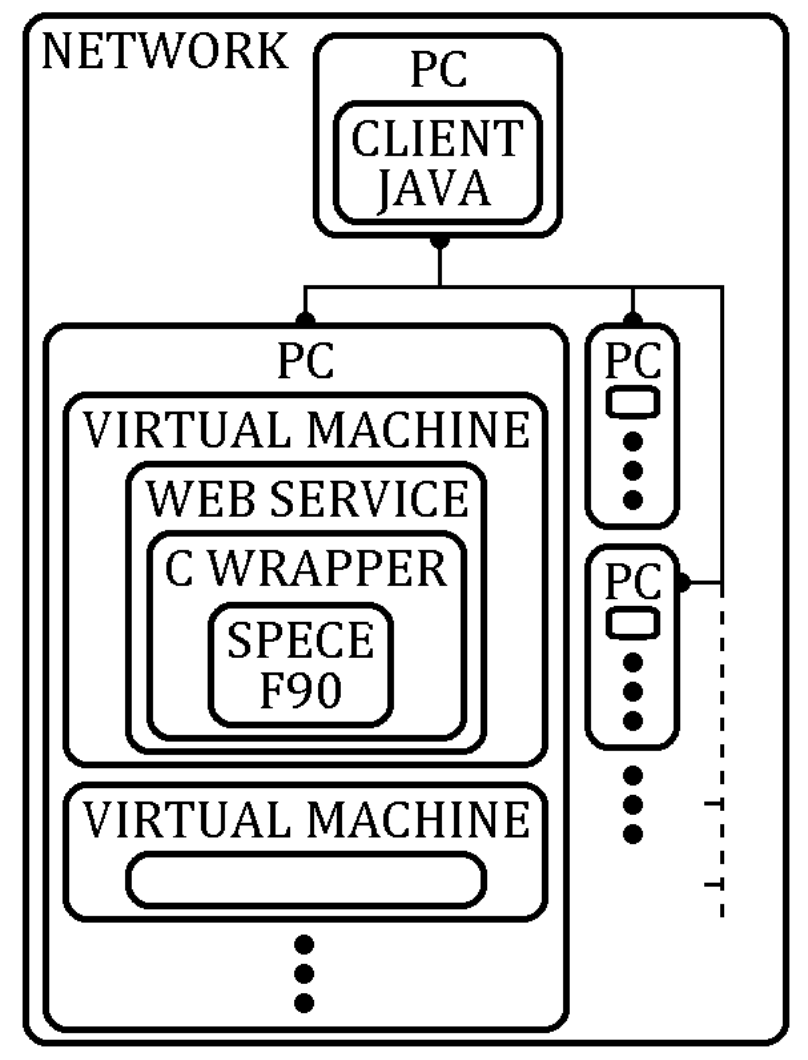

Figure 17. Scheme of possible SPECE parallelisation. A client PC with JAVA analysis software requests predictions from a number of SPECE web services after input parameters are send via the network. To pass this input, a $\mathrm{C}$ wrapper is mandatory for SPECE (F90). Web services can be established on any server-like device, like computational units of a cluster or, here, virtual machines. Dedicated PCs allow multiple virtual machines to operate in parallel.

a means is appreciated which reduces considerably the computational time per prediction. For the latter, a computational parallelisation of the model or machine learning, i.e., a well trained neural network are possible candidates.

For a fusion plasma, kinetic properties of electrons vary spatially captured by continuous temperature and density profiles. Hence, the number of parameters, approximating the profiles, is somewhat fixed, so that any model prediction is meaningful. Furthermore, the measured spectra, and, thus, the number of data points are determined by the diagnostic system.

For the probabilistic analysis presented in this work, the limiting factor time-wise is a SPECE prediction for a broadband spectrum. If carried out by one computational unit, an accurate prediction takes $5 \mathrm{~s}$ or more which would make a probabilistic approach entirely unfeasible. This obstacle is bypassed partly via a parallelisation of SPECE, enabling some investigations of the probabilistic problem.

\section{Implemented Solution}

The basic concept for the parallelisation uses multiple independent instances of SPECE, so that each predicts a sub spectrum for a mutual exclusive set of frequencies. This concept diverts the computational demand on a single PC to a network of PCs or computational units. Thus, the computational time for a single prediction can benefit from a network with high data transfer rate and from well equipped PCs.

One of the tested solutions, relying on the basic concept, follows the scheme shown in figure 17 . The inference for the free parameters is performed by a single PC, immersed in a network of computers. This single PC is a client of a number of independent servers, providing each a SPECE web service. Each server is a virtual machine, and a multi-core PC hosts several virtual machines simultaneously. Thereby, the number of servers can be increased by the usage of several dedicated PCs. Below, details are presented for the achieved parallelisation.

\section{a. JAVA Client}

The probabilistic analysis is implemented into JAVA and is performed on a single PC. JAVA allows multithreading; a way to execute autonomous requests to web services in parallel. For common quantities like the free parameters but for distinct spectral domains, available SPECE web services are contacted via their associated URLs. Each service responds after its SPECE instance has ended the simulation of a sub spectrum. All sub spectra are combined, according to frequency, on the client side, and the result forms one predicted spectrum.

\section{b. Web Service}

Since JAVA cannot communicate directly with Fortran used to implement SPECE, a C wrapper was written which acts as a middleware between the client and SPECE. The $\mathrm{C}$ and Fortran source codes are compiled to a shared library specific for the processor architecture (32-Bit, 64-Bit, ARM).

The web service itself is created with JAVA and is deployed with Tomcat on each server at a specified port. The web service was published and successfully tested on different operating systems like Scientific Linux 6 (32bit, JAC former cluster at JET) and 7 (64-Bit, FREIA current cluster at JET), and Raspbian GNU/Linux 9 (64-/32-bit, ARMv8-A, Raspberry $\mathrm{Pi}^{52}$ ).

\section{c. Network Considerations}

Some challenges arise from the need to use a network by which computational resources are shared with other users. One limiting factor for the computational time is 
the effective data transfer rate determined by the client PC, the network and web service host machines. With rising number of web services, more information is sent per single SPECE prediction via the network. Especially, the transfer of one quantity is the problem; the flux surface geometry in form of a matrix with several thousands entries, amounting to the size of the order of $1 \mathrm{MB}$. While nowadays the standard rate for PC ethernet is Gbit/s, a network with the rate of $100 \mathrm{Mbit} / \mathrm{s}$ slows down the computational time when several tens of web services are used. On the contrary, the on-site network at JET benefits from $1 \mathrm{Gbit} / \mathrm{s}$. To extend the functioning of the parallelisation to the rate $100 \mathrm{Mbit} / \mathrm{s}$, the matrix for the flux surface geometry can be stored locally on the hard disk or SD card of the web service host machine and is read in for each prediction.

A second limitation is the usage of the web service host machines by other users for unrelated computations. A computer cluster like FREIA is designed to perform longterm (several hours) parallel computing for many users managed by a job or batch queue scheduler software. The scheduler determines free computational resources of the cluster and distributes jobs accordingly. This scheduler might interpret cluster nodes (individual computational units) with active SPECE web services to be idle between subsequent requests for a predicted spectrum. If so, the nodes become heavily occupied with operations of other users, slowing down tremendously the computational time. Hence, the web service approach would fail under these conditions. To prevent from continuous failing, it was decided for this work to use dedicated PCs of colleagues to host several virtual machines, having each a SPECE web service.

\section{PCs Hosting Virtual Machines Hosting Web Services}

The solution presented below follows closely the scheme presented in figure 17. Three PCs are used, and each has a quad-core processor with clock frequency of $3.6 \mathrm{GHz}$ or higher, at least $16 \mathrm{~GB}$ RAM, an ethernet data transfer rate of $1 \mathrm{Gbit} / \mathrm{s}$ and a Windows 10 operating system. The PCs are connected via a $100 \mathrm{Mbit} / \mathrm{s}$ network which limits the overall data transfer rate.

The software VMware workstation ${ }^{53}$ enables the parallel availability of several operating systems, so-called virtual machines (VMs), on an individual PC. After setting up the master copy VM with the operating system Scientific Linux 7 as a server, copies can be made to multiply the number of SPECE web services. After tests, it was chosen to establish $8 \mathrm{VMs}$ per host $\mathrm{PC}$ and to assign to each VM one processor and 1.5 GB RAM.

Each published web service is available on a fixed port, say 8080, of the individual VM. For network administration purposes, all VMs hosted by one PC need to share the same IP address inside the network. Thus, network address translation (NAT) is used, so that a unique port of the host PC is forwarded to port 8080 of each VM.

\begin{tabular}{|l|l|l|l|l|l|l|l|l|}
\hline & $1 \mathrm{PC}$ & $1 \mathrm{PC}$ & $1 \mathrm{PC}$ & $1 \mathrm{PC}$ & $2 \mathrm{PCs}$ & $3 \mathrm{PCs}$ & $1 \mathrm{RP}$ & $2 \mathrm{RPs}$ \\
\hline$n_{W S}$ & $1 \times 1$ & $1 \times 2$ & $1 \times 4$ & $1 \times 8$ & $2 \times 8$ & $3 \times 8$ & $1 \times 1$ & $2 \times 1$ \\
\hline$t_{C}(\mathrm{~s})$ & 6.1 & 3.1 & 1.8 & 1.5 & 0.9 & 0.7 & 64.7 & 34.6 \\
\hline$t_{C f}(\mathrm{~ms})$ & 48.1 & 24.8 & 14.4 & 12.0 & 7.0 & 5.2 & 513.2 & 274.4 \\
\hline
\end{tabular}

TABLE III. Computational time $t_{C}$ for single prediction (single-ray) by SPECE parallelised via $n_{W S}$ web services. The prediction of a broadband spectrum $(51.23-278.13 \mathrm{GHz})$ at 126 equidistant frequency nodes was carried out by one, two or three PCs, having each established up to 8 web services. An alternative approach exploits one or two Raspberry $\mathrm{Pi}$ $(\mathrm{RP})$ devices, providing each one web service. The time $t_{C f}$ characterises the duration per single frequency prediction.

\section{a. Investigation of Computational Time}

For the above hardware choice, the computational time $t_{C}$ per single prediction of a broadband spectrum is investigated, depending on the number $n_{W S}$ of used SPECE web services. Despite the PC solution, this speed test includes the results for the SPECE web service established on one or two Raspberry Pis (quad-core, clock frequency $1.4 \mathrm{GHz}, 1 \mathrm{~GB}$ RAM, ethernet data transfer rate 1 Gbit/s). The speed test has been performed when the network was not occupied by other users.

For the parameter set which gives the MAP in Sec. VID, a spectrum is evaluated at 126 frequencies in the range from 51.23 to $278.13 \mathrm{GHz}$ with constant increment for the diagnostic MIX. Furthermore, the Gaussian beam is approximated with the central ray, and, hence, this speed test applies to a single-ray trajectory only.

Table III lists the average values of $t_{C}$. For one PC, $t_{C}$ varies inversely with the number of web services employed, and the duration is reduced from $6.1 \mathrm{~s}$ (single service) to $1.5 \mathrm{~s}$ (8 services). With 24 services (3 PCs), $t_{C}$ settles at $0.7 \mathrm{~s}$ which is an improvement of about one order of magnitude. Too few PCs have been used to determine whether the inverse relation for one $\mathrm{PC}$ continues or the slightly different hardware of each PC masks a linear correspondence between $t_{C}$ and the number of web services. The above applies also for $t_{C f}=t_{C} / 126$ (time per frequency) which is reduced from 48.1 to $5.2 \mathrm{~ms}$.

The alternative choice, relying on Raspberry Pis, is slower by a factor of 10 , at least.

\section{Appendix D: ECE Diagnostic Models}

\section{Convolution Function for MIX and MIO}

Both interferometers probe an interference pattern dependent on the optical path difference $x$ for a finite spatial domain. This implies that the number of measured Fourier coefficient is limited which translates to a spectral convolution function. To act on the shape of the convolution function, the multiplication of a window function $W(x)$ to the data can be carried out. The corresponding 
convolution function is evaluated by

$$
\begin{aligned}
& \operatorname{Conv}\left(f, f^{\prime}\right)=\int W\left[\cos \left(2 \pi \frac{f}{c} x\right)+\sin \left(2 \pi \frac{f}{c} x\right)\right] \\
& \times\left[\cos \left(2 \pi \frac{f^{\prime}}{c} x\right)+\sin \left(2 \pi \frac{f^{\prime}}{c} x\right)\right] d x .
\end{aligned}
$$

The integration is carried out for the cosine with the difference frequency $f^{\prime}-f$ in its argument and for the sine, varying in the sum frequency $f^{\prime}+f$.

When the spectrum $S\left(f^{\prime}\right)$ is evaluated, using the normalised quantity $\operatorname{Conv}_{N}\left(f, f^{\prime}\right)=\operatorname{Conv}\left(f, f^{\prime}\right) /\left(\int \operatorname{Conv}\left(f, f^{\prime}\right) d f\right)$, then all spectra

$$
S\left(f^{\prime}\right)=\int S(f) \operatorname{Conv}_{N}\left(f, f^{\prime}\right) d f
$$

on the right-hand side are solutions to the problem, because the convolution filter removes non-probed Fourier coefficients from any spectrum $S(f)$.

For MIX and MIO, data points which locate outside the spatial domain from $-L_{D S}=-5.12 \mathrm{~mm}$ to $L_{D S}+$ $L_{S S}=26.4 \mathrm{~mm}$ are rejected by the standard analysis procedure, and the window function

$$
W(x)= \begin{cases}\frac{x+L_{D S}}{2 L_{D S}} & :|x| \leq L_{D S} \\ \cos \left(\pi \frac{x-L_{D S}}{2 L_{S S}}\right) & : L_{D S}<x \leq L_{D S}+L_{S S} \\ 0 & : \text { otherwise }\end{cases}
$$

is multiplied to the remaining data set. In the doublesided spatial domain given by the interval $\left[-L_{D S}, L_{D S}\right]$, a ramp is applied. The data located in the single-sided domain is scaled with the first quarter period of a cosine to damp out higher Fourier coefficients. For this window function, the spatial integral for $f^{\prime}+f$ is important below $20 \mathrm{GHz}$. Above this frequency, the cosine integral

$$
\begin{aligned}
& \operatorname{Conv}\left(f^{\prime}-f\right)=L_{D S} \operatorname{sinc}\left(2 \pi \frac{f^{\prime}-f}{c} L_{D S}\right) \quad(\mathrm{D} 4) \\
+ & \frac{L_{S S}}{2}\left[\cos \left(\frac{a}{2}\left(2 L_{D S}+L_{S S}\right)-\frac{\pi}{2} \frac{L_{D S}}{L_{S S}}\right) \operatorname{sinc}\left(\frac{a L_{S S}}{2}\right)\right] \\
+ & \frac{L_{S S}}{2}\left[\cos \left(\frac{b}{2}\left(2 L_{D S}+L_{S S}\right)-\frac{\pi}{2} \frac{L_{D S}}{L_{S S}}\right) \operatorname{sinc}\left(\frac{b L_{S S}}{2}\right)\right]
\end{aligned}
$$

with $a=\pi\left(1+4\left(f^{\prime}-f\right) L_{S S} / c\right) /\left(2 L_{S S}\right)$ and $b=\pi(1-$ $\left.4\left(f^{\prime}-f\right) L_{S S} / c\right) /\left(2 L_{S S}\right)$ approximates the full convolution function very well. Furthermore, the normalisation of $\operatorname{Conv}\left(f^{\prime}-f\right)$ could not be determined analytically but is numerically calculable, yielding $\operatorname{Conv}_{N}\left(f^{\prime}-f\right)$.

\section{Standard Cross-Calibration of HR}

From a scientific point of view, the auxiliary-heating phase during a discharge is most valuable. In order to have the heterodyne radiometer with excellent temporal and spectral resolutions operational, the standard cross-calibration relies on data acquired during the Ohmic-heating phase. This phase has low and intermediate density and temperature, so that HR should work in the linear regime for the channel response (see equation (29)). Several simplifications and assumptions are made which limit the applicability of the determined calibrations $C^{X}$ and $C^{O}$. Formula-wise, the standard calibration procedure is investigated below for $C^{X}$. Though, systematic effects are pointed out for $C^{X}$ and $C^{O}$.

\section{a. Linear Model}

For the signals $V_{H R}^{X}$, a linear dependency is assumed on the ECE spectra incident on the diagnostic antenna. This assumption should be valid for an Ohmic plasma which has low or intermediate electron density and temperature. Hence, the ECE spectra in terms of intensity are small enough in amplitude, and proper settings of the radiometer hardware ensures that each channel operates in the regime of linear response.

During the auxiliary-heating phase, the linearity assumption might not hold. In such a case, the spectra determined with HR and MIX show discrepancies. This is resolved by estimating another set of linear response factors for the radiometer channels, including implicitly the non-linear effect, so that the discrepancies are removed for the auxiliary-heating phase. As a consequence, the discrepancies are shifted to the Ohmic phase for related channels.

\section{b. Offset}

The voltage offset of a channel is estimated from data taken before the plasma is present but not after. Assuming that the result $\bar{V}_{O f f}^{X}$ remains constant during a JET pulse gives the signal difference $\Delta V_{H R}^{X}=V_{H R}^{X}-\bar{V}_{O f f}^{X}$ caused by electron cyclotron emission during plasma operation.

\section{c. Neglecting Cross-Polarisation}

The cross-polarisation terms $S^{O X}$ and $S^{X O}$ are assumed to vanish. Then, the calibration is estimated like

$$
\begin{aligned}
C^{X} & =\frac{\Delta V_{H R}^{X}}{A^{X}\left(T_{R a d A}^{H R X} \cos ^{2} \beta+T_{R a d A}^{H R O} \sin ^{2} \beta\right)} \\
& =S^{X}\left[1+\frac{S^{O X}}{S^{X}} \frac{T_{R a d A}^{H R X} \sin ^{2} \beta+T_{R a d A}^{H R O} \cos ^{2} \beta}{T_{\text {RadA }}^{H R X} \cos ^{2} \beta+T_{\text {RadA }}^{H R O} \sin ^{2} \beta}\right]
\end{aligned}
$$

at a given point in time. The second row in the above equation follows after inserting the related expression (29), neglecting the noise. Only if $S^{O X}$ really vanishes, the standard estimation of $C^{X}$ would be correct. Furthermore, the mismatch stated by the angle $\beta$ and the O-mode contribution depend on plasma parameters and time. Thus, the use of $C^{X}$ is limited to similar discharge parameters. 
Typically, the systematic uncertainty on $C^{X}$ are sufficiently small, so that no heavy discrepancy is at hand during the auxiliary-heating phase. On the contrary, if mainly O-mode contribution is probed, then $C^{O}$ determined accordingly can lead to unacceptable discrepancies. This is believed to be caused by the elevated attenuation (factor 2, see figure $15(\mathrm{~b}) \mathrm{in}^{42}$ ) by the transmission line, so that the ratio $S^{X O} / S^{O}$ becomes relevant.

\section{d. Convoluted Spectra as Reference}

The actual derivation of $C^{X}$ uses reference spectra determined with MIX, because this cornerstone diagnostic is absolutely calibrated and responses linearly up to at least $10 \mathrm{keV}$. Basically, the approximation $T_{R a d A}^{H R X} \cos ^{2} \beta_{H R}+T_{R a d A}^{H R O} \sin ^{2} \beta_{H R} \approx T_{R a d}^{M I X}$ is made in the denominator of equation (D5). While the mismatches of both diagnostics are usually very similar (see figure 3), two essential features remain to be investigated which can cause an over- or underestimation of the calibration.

When diagnostics have separated vacuum lines of sight, the plasma is probed differently. Especially, the central plasma domain might be missed by one of the two diagnostics. Furthermore, refraction affects each diagnostic differently but is minimal during the Ohmic-heating phase.

Another reason to perform the calibration within the Ohmic-heating phase originates in the important condition that the electron temperature and density profiles have no steep gradients. Otherwise, steep gradients in the spectra would occur which cannot be resolved with MIX due to the inherent convolution (see Sec. IV D1). If the profile gradients are small, then $T_{R a d}^{M I X}$ resembles well enough the actual spectrum incident on the radiometer antenna .

\section{e. Reducing Systematic Uncertainties}

To reduce the aforementioned possible systematics, the calibration is carried out for a long duration of the Ohmic-heating phase. Basically, an averaging over several systematic effects takes place. While the measurement noise contributes a negligible portion to the uncertainty, the fundamental difference between $S^{X(O)}$ and $C^{X(O)}$ determines the uncertainty.

\section{Investigation of Sensitivity Matrix S}

The linear model expressed by equation (29) includes the sensitivity matrix $\mathbf{S}$ defined by equation (30). For a given point in time, the entries of $\mathbf{S}$ cannot be determined independently, even if all other quantities are known. This is tackled by the standard cross-calibration (see above in this Section) by assuming explicitly that the off-diagonal elements of $\mathbf{S}$ vanish. There is a more general approach possible described below for the parameters $S^{X}$ and $S^{O X}$, and an example is given. However, future investigations are elementary regarding the underestimated posterior uncertainties on $S^{X}$ and $S^{O X}$.

\section{a. Temporal Model and Linear Gaussian Inversion}

For a single channel probing mainly in X-polarisation, the signal increase caused by the plasma may be given by $\Delta V_{H R}^{X}$ dependent on time $t$. Then, the time dependent model can be obtained by taking the transpose of equation (29) applied for each time and focussing on the detected X-mode component on the left-hand side. This gives

$$
\Delta \vec{V}_{H R}^{X}=\left(\begin{array}{c}
\Delta V_{H R}^{X}\left(t_{1}\right) \\
\Delta V_{H R}^{X}\left(t_{2}\right) \\
\vdots
\end{array}\right)=A^{X}\left(\begin{array}{c}
\mathbf{M}_{\beta}\left(t_{1}\right) \vec{T}_{R R A}^{H R}\left(t_{1}\right) \\
\mathbf{M}_{\beta}\left(t_{2}\right) \vec{T}_{R a d A}^{H R}\left(t_{2}\right) \\
\vdots
\end{array}\right)\left(\begin{array}{c}
S^{X} \\
S^{O X}
\end{array}\right)+\left(\begin{array}{c}
\mathcal{N}\left(0,\left(\sigma_{H R}^{X}\left(t_{1}\right)\right)^{2}\right) \\
\mathcal{N}\left(0,\left(\sigma_{H R}^{X}\left(t_{2}\right)\right)^{2}\right) \\
\vdots
\end{array}\right)=\mathbf{M}_{t} \vec{S}^{X}+\mathcal{N}\left(\overrightarrow{0}, \boldsymbol{\Sigma}_{H R}^{X}\right)
$$

with the data vector on the left-hand side, the time dependent mapping matrix $\mathbf{M}_{t}$ and the multi-variate normal distribution with vanishing mean and diagonal data covariance matrix $\boldsymbol{\Sigma}_{H R}^{X}$. Hence, one can state a likelihood by

$$
\mathcal{L} \propto \exp \left(-\frac{1}{2}\left[\Delta \vec{V}_{H R}^{X}-\mathbf{M}_{t} \vec{S}^{X}\right]^{T}\left(\boldsymbol{\Sigma}_{H R}^{X}\right)^{-1}\left[\Delta \vec{V}_{H R}^{X}-\mathbf{M}_{t} \vec{S}^{X}\right]\right) .
$$

Choosing a Gaussian prior for $S^{X}$ and $S^{O X}$ enables the exploitation of a standard linear and analytical inversion technique. Then, the Gaussian posterior for the parameters of interest becomes

$$
p\left(S^{X}, S^{O X} \mid \mathbf{M}_{t}, \boldsymbol{\Sigma}_{H R}^{X}, \vec{V}_{H R}^{X}\right)=\mathcal{N}\left(\vec{S}_{P o}^{X}, \boldsymbol{\Sigma}_{P o, S}\right)
$$

with the posterior mean $\vec{S}_{P o}^{X}=\boldsymbol{\Sigma}_{P o, S} \mathbf{M}_{t}^{T}\left(\boldsymbol{\Sigma}_{H R}^{X}\right)^{-1} \vec{V}_{H R}^{X}$, having chosen a vanishing prior mean, and with the posterior covariance $\boldsymbol{\Sigma}_{P o, S}=\left(\mathbf{M}_{t}^{T}\left(\boldsymbol{\Sigma}_{H R}^{X}\right)^{-1} \mathbf{M}_{t}+\boldsymbol{\Sigma}_{P r, S}^{-1}\right)^{-1}$ for the prior covariance $\boldsymbol{\Sigma}_{P r, S}$. 


\section{b. Brief Example}

To demonstrate the above approach, some additional steps are mandatory to bring JET data in the proper format.

The voltage offset and its uncertainty are estimated from pre-pulse data. The data acquired from $3 \mathrm{~s}$ to $7 \mathrm{~s}$ during the Ohmic phase of JET pulse 92436 is used to compare the results with $C^{X}$ obtained with the standard cross-calibration. The radiative temperatures, detected by MIX and MIO at a time $t$ over the duration of $16.7 \mathrm{~ms}$, are used to approximate the quantity coupled to the transmission line of the radiometer. Accordingly, $\mathbf{M}_{\beta} \vec{T}_{R a d A}^{H R} \approx\left(T_{R a d}^{M I X}, T_{R a d}^{M I O}\right)^{T}$ is set. Thereby, $T_{R a d}^{M I X}$ and $T_{\text {Rad }}^{M I O}$ are evaluated by linear interpolation at the radio frequency of each HR channel. Furthermore, the signal difference $\Delta \vec{V}_{H R}^{X}$ is temporally averaged over each available time window with the duration $16.7 \mathrm{~ms}$. To estimate $\Sigma_{H R}^{X}$, the standard deviation of the mean of the signal difference and the uncertainty of the voltage offset are quadratically added (Gaussian uncertainty propagation). The diagonal prior covariance $\boldsymbol{\Sigma}_{P r, S}$ has large entries like for example $100^{2}(\mathrm{~V} / \mathrm{keV})^{2}$.

The found posterior means $S_{P_{o}}^{X}$ and $S_{P_{o}}^{O X}$ are depicted in the top of figure 18. Since $S_{P_{o}}^{X}>S_{P_{o}}^{O X}$ holds, the estimated cross-polarisation seems to be in the right order of magnitude. Furthermore, $S_{P o}^{X}$ and the calibration $C^{X}$ are similar. The predictions, for the standard calibration model with $C^{X}$ and for the alternative model with $S_{P_{o}}^{X}$ and $S_{P o}^{O X}$, follow the trend of the measured data equally well. Given these findings, it is astonishing that the cross-polarisation is estimated to be as large as 20 to $30 \%$ of the value of $S_{P o}^{X}$. Furthermore, a negative cross-polarisation is found for the spectral domain from $127.825 \mathrm{GHz}$ to $134.5 \mathrm{GHz}$. This is caused by missing out the non-linearity in the model revealed by a more detailed investigation.

The posterior standard deviations of the parameters $S^{X}$ and $S^{O X}$ are small when related to the actual mean values. However, comparing predictions with the measured data shows that the posterior standard deviations are underestimated by at least one order of magnitude. Systematic deviations from the model used are identified as the reasons. For example, the plasma position varies horizontally, and, thus, the approximation $\mathbf{M}_{\beta} \vec{T}_{R a d A}^{H R} \approx\left(T_{R a d}^{M I X}, T_{R a d}^{M I O}\right)^{T}$ is too crude. Hence, an additional contribution to the data covariance should be included and estimated, so that missed systematic effects enter in the uncertainties of the parameters of interest.

For almost all HR channels, the posterior correlation coefficient $\rho_{P o}$ settles close to -1 . Hence, the inferred quantities $S^{X}$ and $S^{O X}$ are anti-correlated, since their contributions are additive to model the data.

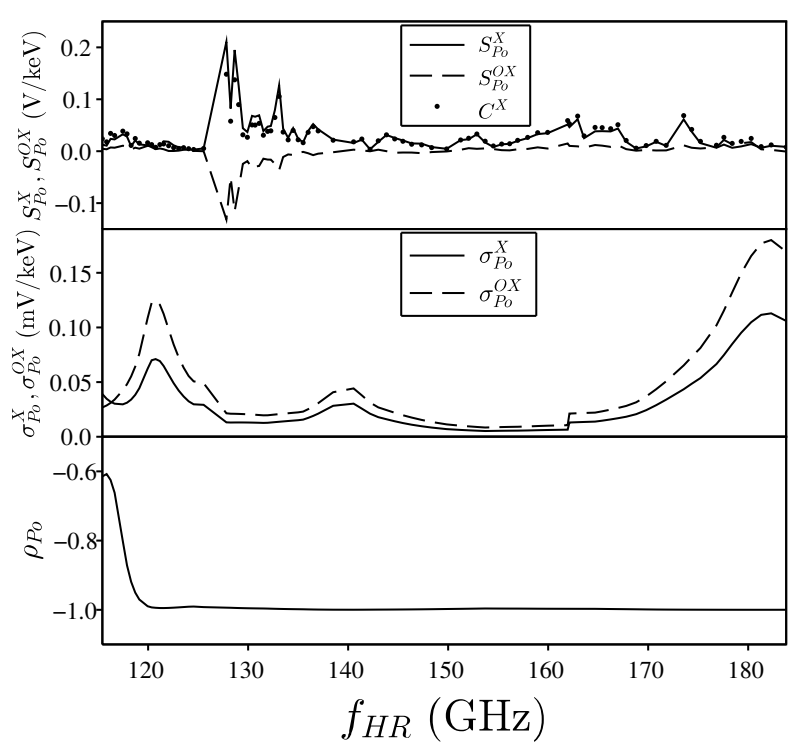

Figure 18. Example to estimate heterodyne radiometer (HR) sensitivity $S^{X}$ and cross-polarisation $S^{O X}$ (due to transmission line) using linear Gaussian inversion. Ohmic plasma data of JET pulse 92436 was used for the duration from $3 \mathrm{~s}$ to $7 \mathrm{~s}$. Top: Posterior mean values (solid and dashed). $S_{P_{o}}^{X}$ is comparable with the results $C^{X}$ (dots) of the standard calibration procedure. $S_{P_{o}}^{O X}$ remains finite but is usually smaller in magnitude than $S_{P o}^{X}$. The negative values of $S_{P o}^{O X}$ in the range $127.825-134.5 \mathrm{GHz}$ are believed to originate in a non-linear behaviour. Middle: Posterior standard deviations. In principle, small values can be obtained. However, systematic effects, like the impact of the vertical movement of the plasma, are not taken into account. They are expected to be larger by one order of magnitude, at least. Bottom: Posterior correlation $\rho_{P o}$ between $S^{X}$ and $S^{O X}$. Since both quantities enter in distinct summands to model the data, an anti-correlation is found in general. The deviating behaviour below $120 \mathrm{GHz}$ is believed to be caused by having not chosen properly the time window for this spectral domain, since some HR signals are partly vanishing.

Appendix E: $\psi_{N}$ vs. $|\vec{B}|$

While the flux surface geometry seems to be similar in real-space coordinates for LFS and HFS, a major difference arises when the flux surface label is presented versus the magnitude $|\vec{B}|$ of the magnetic field strength along a radial line of sight. This difference comes with the dependency of $|\vec{B}|$ on the major radius $R$, affecting $\psi_{N}(|\vec{B}|)$ differently on the LFS and HFS.

For a tokamak like JET with large aspect ratio, the toroidal magnetic field contributes most to $|\vec{B}|$ and can be approximated by $B_{\Phi V a c} R_{M A} / R$ given the vacuum magnetic field $B_{\Phi V a c}$ at the major radius $R_{M A}$ of the magnetic axis. For the variation of $\psi_{N}(|\vec{B}|)$ along a central line of sight from plasma axis to the edge, a zero order 
estimation gives

$$
\left|\frac{\Delta \psi_{N}}{\Delta|\vec{B}|}\right|_{\substack{L F S \\ H F S}}= \pm \frac{1}{B_{\Phi V a c} R_{M A}} \frac{R_{M A} R_{H F S}}{R_{H F S}-R_{M A}}
$$

for the LFS and HFS, respectively. For a typical JET example, $|\vec{B}|=2.6 \mathrm{~T}$ is present at the magnetic axis $\left(R_{M A} \approx 3 \mathrm{~m}\right)$, and one has $|\vec{B}| \approx 1.95 \mathrm{~T}$ at $R_{L F S} \approx 4 \mathrm{~m}$ and $|\vec{B}| \approx 3.9 \mathrm{~T}$ at $R_{H F S} \approx 2 \mathrm{~m}$. Hence, one finds that the global variation of $\psi_{N}(|\vec{B}|)$ on the LFS is twice as high as the one on the HFS.

\section{ACKNOWLEDGEMENTS}

The authors would like to express their gratitude towards A. Simonetto for technical support, P. Platania and S. Garavaglia for making available their office PCs to achieve a more effective SPECE parallelisation.

The first author thanks the institutions ISTP (Milan, Italy), KTH (Stockholm, Sweden), CCFE (Abingdon, United Kingdom), IPP (Greifswald and Garching, Germany) for their patience and continuous scientific support.

The first author acknowledges the patience, the moral support, the help and the trust of his wife, C. Marchetto, and bows to her for giving birth to our twins. May the sun and the star be bright lights in our family.

The thoughts of the first author are with his parents, Sabine and Karl-Heinz, and his grandparents, Helga and Siegfried, whose health is affected by severe diseases.

The first author is humbled by the great achievements of the European peoples, ensuring long-lasting peace, freedom of movement and international collaborations. For instance, the reunification of Germany was the historical foundation of the first author's vita like for many others of his generation. May future generations benefit from the same spirit that we are more united than divided.

This work has been carried out within the framework of the EUROfusion Consortium and has received funding from the Euratom research and training programme 2014-2018 and 2019-2020 under grant agreement No 633053. The views and opinions expressed herein do not necessarily reflect those of the European Commission.

\section{REFERENCES}

\footnotetext{
${ }^{1}$ T.H. Stix, "Highlights in Early Stellarator Research at Princeton", J. Plasma Fusion Res. SERIES, 1, 3 (1998).

${ }^{2}$ V.P. Smirnov, "Tokamak foundation in USSR/Russia 1950-1990", Nucl. Fusion, 50, 014003 (2010).

${ }^{3}$ T. Donné et al., "European Research Roadmap to the Realisation of Fusion Energy", https://www.euro-fusion.org/fileadmin/ user_upload/EUROfusion/Documents/2018_Research_roadmap_ long_version_01.pdf

${ }^{4}$ X. Litaudon et al., "Overview of the JET results in support to ITER", Nucl. Fusion, 57, 102001 (2017).
}

${ }^{5}$ R.C. Wolf et al., "Major results from the first plasma campaign of the Wendelstein 7-X stellarator", Nucl. Fusion, 57, 102020 (2017).

6ITER Organization, "ITER Research Plan within the Staged Approach (Level III - Provisional Version)", https : //www.iter.org/doc/www/content/com/Lists/ITER\% 20Technical\%20Reports/Attachments/9/ITER-Research-Plan_ final_ITR_FINAL-Cover_High-Res.pdf, Report No. ITR-18-003 (2018).

${ }^{7}$ G. Federici et al., "Overview of the DEMO Design-Staged Approach in Europe", Nucl. Fusion, to be published in 58, (2018).

${ }^{8}$ A. E. Costley, "50 years of electron cyclotron emission research", Fusion Sci. Technol., 55, 1 (2009).

${ }^{9}$ D. Dodt et al., "Improved framework for the maintenance of the JET intershot analysis chain", Fusion Eng. Des., 88, 79 (2013).

${ }^{10}$ M. Romanelli et al., "JINTRAC: A System of Codes for Integrated Simulation of Tokamak Scenarios", Plasma Fusion Res., 9, 3403023 (2014).

${ }^{11}$ B. A. Grierson et al., "Orchestrating TRANSP Simulations for Interpretative and Predictive Tokamak Modeling with OMFIT", Fusion Sci. Technol., 74, 101 (2018).

${ }^{12}$ M. Irishkin et al., "Applications of Bayesian temperature profile reconstruction toautomated comparison with heat transport models and uncertaintyquantification of current diffusion", Fusion Eng. Des., 100, 204 (2015).

${ }^{13} \mathrm{~V}$. Dose et al., "Tokamak edge profile analysis employing Bayesian statistics", Nucl. Fusion, 41, 1671 (2001).

${ }^{14}$ R. Fischer et al., "Probabilistic lithium beam data analysis", Plasma Phys. Control. Fusion, 50, 085009 (2008).

${ }^{15}$ S. K. Rathgeber et al., "Estimation of edge electron temperature profiles via forward modelling of the electron cyclotron radiation transport at ASDEX Upgrade", Plasma Phys. Control. Fusion, 55, 025004 (2013).

${ }^{16}$ R. Fischer et al., "Thomson scattering analysis with the Bayesian probability theory", Plasma Phys. Control. Fusion, 44, 1501 (2002).

${ }^{17}$ J. Svensson et al., "Integrating diagnostic data analysis for W7AS using Bayesian graphical models", Rev. Sci. Instrum., 75, 4219 (2004).

${ }^{18}$ J. Svensson, A. Werner, and JET EFDA contributors, "Current tomography for axisymmetric plasmas", Plasma Phys. Control. Fusion, 50, 085002 (2008).

${ }^{19}$ O. Ford et al., "Forward modeling of JET polarimetry diagnostic", Rev. Sci. Instrum., 79, 10F324 (2008).

${ }^{20}$ S. Kwak et al., "Bayesian electron density inference from JET lithium beam emission spectra using Gaussian processes", Nucl. Fusion, 57, 036017 (2017).

${ }^{21}$ M. A. Chilenski et al., "Improved profile fitting and quantification of uncertainty in experimental measurements of impurity transport coefficients using Gaussian process regression", Nucl. Fusion, 55, 023012 (2015).

${ }^{22}$ A. Langenberg et al., "Forward Modeling of X-Ray Imaging Crystal Spectrometers Within the Minerva Bayesian Analysis Framework", Fusion Sci. Technol., 69, 560 (2016).

${ }^{23}$ U. Hoefel et al., "Bayesian Modelling of Microwave Radiometer Calibration on the example of the Wendelstein 7-X Electron Cyclotron Emission diagnostic", Rev. Sci. Instrum., 90, 043502 (2019).

${ }^{24}$ J. Svensson, A. Werner, "Large Scale Bayesian Data Analysis for Nuclear Fusion Experiments", IEEE International Symposium on Intelligent Signal Processing (2007).

${ }^{25}$ D. Sivia, J. Skilling, " Data Analysis: A Bayesian Tutorial", Oxford University Press, Great Clarendon Street, Oxford OX2 6DP, United Kingdom (2006).

${ }^{26}$ J. P. Freidberg, "Ideal MHD", Cambridge University Press, University Printing House, Cambridge CB2 8BS, United Kingdom (2014).

27 J. Wesson et al., "Tokamaks", Clarendon Press-Oxford, Great Clarendon Street, Oxford OX2 6DP, United Kingdom (2004).

${ }^{28}$ L. L. Lao et al., "Reconstruction of current profile parameters 
and plasma shapes in tokamaks", Nucl. Fusion, 25, 1611 (1985).

${ }^{29}$ L. L. Lao et al., "Equilibrium analysis of current profiles in tokamaks", Nucl. Fusion, 30, 1035 (1990).

${ }^{30}$ L. L. Lao et al., "MHD Equilibrium Reconstruction in the DIII-D Tokamak", Fusion Sci. Technol., 48, 968 (2005).

${ }^{31} \mathrm{M}$. Gelfusa et al., "Influence of plasma diagnostics and constraints on the quality of equilibrium reconstructions on Joint European Torus", Rev. Sci. Instrum., 84, 103508 (2013).

${ }^{32} \mathrm{E}$. Belonohy et al., "The Effect of the Accuracy of Toroidal Field Measurements on Spatial Consistency of Kinetic Profiles at JET", JET Report, EFDA-JET-CP(14)05/18 (2014).

${ }^{33}$ C. E. Rasmussen and C. K. I. Williams, "Gaussian Processes for Machine Learning", The MIT Press, Cambridge, Massachusetts, USA (2006).

${ }^{34}$ G. Bekefi, "Radiation Processes in Plasmas", John Wiley \& Sons, New York (1966).

${ }^{35}$ D. Farina et al., "SPECE: a Code for Electron Cyclotron Emission in Tokamaks", Proc. of BURNING PLASMA DIAGNOSTICS: An International Conference, Varenna, Italy, 2007, edited by G. Gorini, F. P. Orsitto, E. Sindoni, M. Tardocchi, p. 128.

${ }^{36}$ N.B. Marushchenko et al., "Ray-tracing code TRAVIS for ECR heating, EC current drive and ECE diagnostic", Comput. Phys. Commun., 185, 165 (2014).

${ }^{37} \mathrm{E}$. de la Luna et al., "Impact of bulk non-Maxwellian electrons on electron temperature measurements", Rev. Sci. Instrum., 74, 1414 (2003).

${ }^{38}$ T. Zhou et al., "Synthetic Diagnostic for Interpreting the ECE Spectrum on EAST", Fusion Sci. Technol., 74, 154 (2018).

${ }^{39}$ S. Schmuck et al., "Electron Temperature and Density Inferred from JET ECE Diagnostics", 38th EPS Conference on Plasma Physics, Strasbourg, France (2011).

${ }^{40} \mathrm{~S}$. Schmuck et al., "Bayesian derivation of electron temperature profile using JET ECE diagnostics", 41th EPS Conference on Plasma Physics, Berlin, Germany (2014).

${ }^{41}$ R. Prater, "Heating and current drive by electron cyclotron waves", Phys. Plasmas, 11, 2349 (2014)

${ }^{42} \mathrm{~S}$. Schmuck et al., "Electron cyclotron emission spectra in X- and O-mode polarisation at JET: Martin-Puplett interferometer, absolute calibration, revised uncertainties, inboard/outboard temperature profile, and wall properties", Rev. Sci. Instrum., 87, 093506 (2016).

${ }^{43} \mathrm{M}$. Bornatici et al., "Electron cyclotron emission and absorption in fusion plasmas", Nucl. Fusion 23, 1153 (1983).

${ }^{44}$ G. F. Matthews et al., "JET ITER-like wall - overview and experimental programme", Phys. Scr., T145, 014001 (2011).

${ }^{45}$ A. Sirinelli et al., "Multiband reflectometry system for density profile measurement with high temporal resolution on JET tokamak", Rev. Sci. Instrum., 81, 10D939 (2010).

${ }^{46}$ S. Schmuck et al., "Electron cyclotron emission measurements on JET: Michelson interferometer, new absolute calibration, and determination of electron temperature", Rev. Sci. Instrum., 83, 125101 (2012).

${ }^{47}$ E. de la Luna et al., "Electron Cyclotron Emission Radiometer Upgrade on the Joint European Torus (JET) Tokamak", Rev. Sci. Instrum., 75, 3831 (2004).

${ }^{48}$ S. Schmuck and J. Svensson, "Fourier Spectroscopy: A Bayesian Way", Int. J. Spectrosc., 2017, 9265084 (2017).

${ }^{49}$ H.-J. Hartfuß and T. Geist, "Fusion Plasma Diagnostics with mm-Waves", Wiley-VCH Verlag GmbH \& Co. KGaA, Boschstr. 12, 69469 Weinheim, Germany (2013).

${ }^{50} \mathrm{E}$. Mazzucato, "Microwave reflectometry for magnetically confined plasmas", Rev. Sci. Instrum., 69, 2201 (1998).

${ }^{51}$ O. P. Ford, "Tokamak Plasma Analysis through Bayesian Diagnostic Modelling", PhD Thesis, University of London, Imperial College London, Department of Plasma Physics (2010), http://oliford.co.uk/phys/thesis-final.pdf.

${ }^{52}$ Raspberry Pi 3 Model B+, https://docs-emea.rs-online.com/webdocs/162c/ 0900766b8162cdf1.pdf.

${ }^{53}$ VMware Workstation 14, https://www.vmware.com. 